\title{
Anomalous transport at weak coupling
}

\author{
Subham Dutta Chowdhury and Justin R. David \\ Centre for High Energy Physics, Indian Institute of Science, \\ C.V. Raman Avenue, Bangalore 560012, India \\ E-mail: subham@cts.iisc.ernet.in, justin@cts.iisc.ernet.in
}

ABSTRACT: We evaluate the contribution of chiral fermions in $d=2,4,6$, chiral bosons, a chiral gravitino like theory in $d=2$ and chiral gravitinos in $d=6$ to all the leading parity odd transport coefficients at one loop. This is done by using finite temperature field theory to evaluate the relevant Kubo formulae. For chiral fermions and chiral bosons the relation between the parity odd transport coefficient and the microscopic anomalies including gravitational anomalies agree with that found by using the general methods of hydrodynamics and the argument involving the consistency of the Euclidean vacuum. For the gravitino like theory in $d=2$ and chiral gravitinos in $d=6$, we show that relation between the pure gravitational anomaly and parity odd transport breaks down. From the perturbative calculation we clearly identify the terms that contribute to the anomaly polynomial, but not to the transport coefficient for gravitinos. We also develop a simple method for evaluating the angular integrals in the one loop diagrams involved in the Kubo formulae. Finally we show that charge diffusion mode of an ideal 2 dimensional Weyl gas in the presence of a finite chemical potential acquires a speed, which is equal to half the speed of light.

Keywords: Anomalies in Field and String Theories, Field Theories in Lower Dimensions, Field Theories in Higher Dimensions

ARXIV EPRINT: 1508.01608 


\section{Contents}

1 Introduction 1

2 Anomalies and transport in $d=2 \quad 5$

2.1 Chiral fermions 6

$\begin{array}{lll}2.2 & \text { Chiral bosons } & 16\end{array}$

$\begin{array}{lll}2.3 & \text { Chiral gravitino like system } & 21\end{array}$

3 Anomalies and transport in $d=4 \quad 25$

3.1 Chiral fermions $\quad 26$

4 Anomalies and transport in $d=6 \quad 35$

$\begin{array}{lll}4.1 & \text { Chiral fermions } & 37\end{array}$

4.2 Chiral gravitinos 44

$5 \quad$ Hydrodynamic modes in $d=2$ Weyl gas 46

6 Conclusions $\quad 50$

A Moments of statistical distributions $\quad 50$

B Evaluation of transport coefficients in $d=6 \quad 51$

B.1 Contributions to $\zeta_{1}^{(6)} \quad 51$

B.2 Contributions to $\zeta_{2}^{(6)} \quad 54$

B.3 Contributions to $\zeta_{3}^{(6)} \quad 56$

B.4 Contributions to $\lambda_{3}^{(6)} \quad 59$

\section{Introduction}

The modifications of the macroscopic equations of hydrodynamics due to the presence of quantum anomalies of the underlying theory has been the focus of recent interest. It began with the observation of parity odd terms in the constitutive relation of the charge current in the holographic dual of $\mathcal{N}=4$ super Yang Mills at finite temperature and chemical potential [1-3]. This was then understood from general considerations using the equations of hydrodynamics, anomalous conservation laws in presence of background fields and the second law of thermodynamics [4]. These parity odd transport coefficients are non-dissipative. This fact was used to determine the relation between microscopic anomalies and the macroscopic parity odd transport coefficient using a equilibrium partition function $[5,6]$.

Using general considerations from anomalies and the second law of thermodynamics or using the equilibrium partition function method it is not possible to determine the 
precise numerical constant that determine the relation between the parity odd transport coefficients and the gravitational anomalies or mixed gauge-gravitational anomalies of the microscopic theory. One reason for this is that both these methods rely on performing derivative expansions in velocities. Any relation between the gravitational anomalies or mixed gauge-gravitational anomalies would involve a jump in the derivative expansion. For example consider hydrodynamics in $d=2$, the gravitational anomaly occurs at the 2nd derivative in the conservation law, however it affects the constitutive relations at the zeroth order in the derivative expansion [7]. There are three methods in literature which relates these anomalies to the parity odd transport coefficients. The first is the direct perturbative method of evaluating the transport coefficient of interest using the corresponding Kubo formula. This is usually done for free chiral fermions and then arguing that it is not renormalized, either using holography or in perturbation theory [8-11]. The second method is using the consistency of the Euclidean vacuum, developed by [7, 12]. The third method relies on performing one loop integration to obtain the theory on the spatial slice to relate the the anomalies to the Chern-Simons couplings and then arguing that these are one loop exact $[11,13]$. The Chern-Simons couplings are in turn related to the transport coefficients. ${ }^{1}$ To make this discussion concrete let us consider $d=2$ and the the parity odd term in the constitutive relation for the stress tensor ${ }^{2}$ given by

$$
T^{\mu \nu}=(\epsilon+P) u^{\mu} u^{\nu}-P \eta^{\mu \nu}+\lambda^{(2)}\left(u^{\mu} \epsilon^{\nu \rho} u_{\rho}+u^{\nu} \epsilon^{\mu \rho} u_{\rho}\right) .
$$

Here $u^{\mu}$ is the velocity profile of the fluid. From general considerations of the equilibrium partition function and conservation laws $[14,15]^{3}$ it can be shown that transport coefficient $\lambda^{(2)}$ is of the form

$$
\lambda^{(2)}=\tilde{c}_{2 d} T^{2}-c_{s} \mu_{-}^{2},
$$

where $T$ is the temperature and $\mu_{-}$is the chiral chemical potential. The Euclidean partition method shows that $c_{s}$ is the coefficient of the charge current anomaly defined by the conservation equation

$$
\partial_{\mu} j^{\mu}=c_{s} \epsilon^{\mu \nu} F_{\mu \nu},
$$

$F_{\mu \nu}$ is the background field strength. However the relation between $\tilde{c}_{2} d$ and the pure gravitational anomaly $c_{g}$ defined by

$$
\partial_{\nu} T^{\mu \nu}=F_{\nu}^{\mu} j^{\nu}+c_{g} \epsilon^{\mu \nu} \nabla_{\nu} R
$$

where $R$ is the background curvature cannot be shown using methods which involve expansions in derivatives. This is because the coefficient $\tilde{c}_{2 d}$ occurs at the zeroth order in derivative in the stress tensor. From (1.4) it is seen that pure gravitational anomaly is effective at the 2 nd order in the derivative expansion. It was argued using an argument which involves the consistency of the Euclidean vacuum that

$$
\tilde{c}_{2 d}=-8 \pi^{2} c_{g} .
$$

\footnotetext{
${ }^{1}$ We thank Kristan Jensen and Zohar Komargodski for informing us about this approach.

${ }^{2}$ We have written down the stress tensor in the anomaly frame.

${ }^{3}$ See [16] for an alternative approach.
} 
A similar relation was obtained between the mixed gravitational anomaly and the parity odd transport coefficient in $d=4$ determined by the two point function of the charge current and the stress tensor. In $d=4$ the relation of the type in (1.5) has been verified by a direct perturbative calculation done in [8] as well as using the holographic dual of $\mathcal{N}=4$ Yang-Mills [9]. It was also shown within perturbation theory the relation in (1.5) is not renormalized for theories of chiral fermions which interact via Yukawa couplings. Similar equations relating parity odd transport and anomalies including gravitational anomalies were obtained for arbitrary even dimensions $[7,12,17,18]$ and summarized by the 'replacement rule'. However the relation between gravitational anomalies and transport of the type given in equation (1.5) for dimensions other that of $d=4$ has not yet been verified by a direct perturbative calculation. Further more it has been suspected that higher spin chiral fermions like gravitinos violate the relations of the type $(1.5)[13,19,20]$.

Motivated by these questions we study the relationship between anomalies and parity odd transport coefficients using the direct approach of evaluating the respective Kubo formulae. We consider theories of free chiral fermions in $d=2,4,6$ and chiral bosons in $d=2$ at finite temperature and chemical potential. We evaluate all the leading parity odd transport coefficients. This is done using the method developed by [21] which involves evaluating the Feynman diagrams in finite temperature field theory and then performing the Matsubara sums. This approach is different form that is used in [22] to study transport properties of free chiral fermions in arbitrary even dimensions that uses Schwinger-Keldysh propagators We keep track of contributions that arise from gauge anomalies as well as pure gravitational anomalies and mixed gauge-gravitational anomalies. To address the issue of whether higher spin fermions obey the 'replacement rule' we study the contributions of a chiral gravitino like theory in $d=2$ and chiral gravitinos in $d=6$ to parity odd transport.

We now briefly summarize the results of this work. The Feynman diagrams which contribute to the transport coefficients in $d=2$ are considerably different from that in higher dimensions. This is because the spin connection is not present in the action of fermions in $d=2$. From the perturbative calculation in $d=2$ we show that chiral fermions as well as chiral bosons obey the relation in (1.5) predicted by the argument involving the consistency of the Euclidean vacuum. The transport coefficients of the chiral bosons is identical to that of chiral fermions which is expected from Bose-Fermi duality in $d=2$. For a higher spin fermion, that is a gravitino like theory, we show that the the relation in (1.5) does not hold by an explicit perturbative analysis. In $d=4$ we reproduce the results of $[8,21,22]$ and show the perturbative results for the transport coefficients agree with the 'replacement rule'. Since gravitinos are not charged, they do not contribute to the mixed anomaly in $d=4$ and they do not contribute to any of the parity odd transport coefficients as well. In $d=6$, for chiral fermions we reproduce the results of [22] for the contributions from the gauge anomaly to parity odd transport. We also keep track of contributions from gravitational anomalies. We show that contributions of chiral fermions to parity odd transport from the pure gravitational and the mixed anomaly agree with that predicted by the argument involving the consistency of the Euclidean vacuum. Next we consider gravitinos in $d=6$ and show that its contribution to parity odd transport is not related to the pure gravitational anomaly and therefore does not agree with the prediction obtained from the replacement rule. 
From out study of higher spin chiral fermions in $d=2$ and $d=6$ we can draw the following general lesson for their contribution to parity odd transport. These transport coefficients arise in correlators involving only stress energy tensor. We see that the reason that gravitinos do not obey the 'replacement rule' is because of the presence of the following term in their stress energy tensor.

$$
T_{\mu \nu}^{(3 / 2) e x t r a}=-\frac{i}{2}\left[\partial_{\sigma}\left(\bar{\psi}^{\sigma} \gamma_{\mu} \psi_{\nu}-\bar{\psi}_{\mu} \gamma_{\nu} \psi^{\sigma}\right)+(\mu \leftrightarrow \nu)\right] .
$$

This arises from the linearization of the Christoffel connection in the kinetic term for the gravitinos which is not present for fermions. Such a term gives rise to the interaction between the magnetic moment of the gravitino with the gravitational field [23]. It is this term that modifies the anomaly polynomial for gravitinos from that of a chiral spinor. However on evaluating Kubo formulae, at the zero frequency and zero momentum limit the contribution of the extra term in (1.6) vanishes. Thus the contribution of the gravitino to the transport coefficient is proportional to the chiral spinor. In fact it is just $d-1$ times that of of the chiral spinor. The -1 accounts for the ghosts. We observe this phenomenon for the gravitino like theory in $d=2$ and gravitinos in $d=6$ while evaluating the Feynman diagrams involved in the Kubo formula, and note that this property is independent of dimensions.

Apart form understanding the 'replacement rule' our explicit calculations also point to a technical simplification in evaluating the integrals involved in the one loop diagrams. We see that it is possible to take the zero frequency and zero momentum limit to obtain the transport coefficient before performing the angular integrations involved in the one loop diagrams. We develop a prescription for evaluating these integrals. This simplifies the calculation considerably compared to the ones in [8, 10, 21]. In fact in [22] a guess is made for the angular integrals which was verified case by case. We will see that the angular integrals are elementary after taking the zero frequency and zero momentum limit.

Finally since parity odd transport coefficients appear at the zeroth order in the derivative expansion in $d=2$ they can affect linearized hydrodynamic modes at the leading order. We show that the charge diffusion mode develops a velocity in presence of the parity odd transport coefficients. For the Weyl gas we see that this velocity is $1 / 2$ times the speed of light.

The organization of the paper is as follows. In section 2 we study parity odd transport in $d=2$, we first obtain the relevant Kubo formula for the transport coefficient and then evaluate the contribution of chiral fermions, chiral bosons and a chiral gravitino like theory to the two leading transport coefficients. From the explicit calculation we will see how the 'replacement rule' breaks down for the case of gravitino like theory in $d=2$. In section 3 we look at $d=4$ and repeat the same analysis for chiral fermions. In section 4 we turn to $d=6$ and study parity odd transport of both chiral fermions and chiral gravitinos. Again we see that chiral gravitinos do not obey the replacement rule. In section 5 we study linearized hydrodynamic modes which are modified by parity odd transport coefficients in $d=2$. Appendix A contains details of moments of statistical distributions involved in obtaining the transport coefficients. Appendix B contains the details of the calculations of all the leading parity odd transport in $d=6$. 


\section{Anomalies and transport in $d=2$}

In this section we first evaluate the 2 leading parity odd transport coefficients. They occur at the zeroth order in the derivative expansion in the velocities. To obtain the Kubo formulae for these transport coefficients, let us first write down the constitutive relations to the zeroth order in derivatives. The stress tensor and the current are given by ${ }^{4}$

$$
\begin{aligned}
T^{\mu \nu} & =(\epsilon+P) u^{\mu} u^{\nu}-P \eta^{\mu \nu}+\lambda^{(2)}\left(u^{\mu} \epsilon^{\nu \rho} u_{\rho}+u^{\nu} \epsilon^{\mu \rho} u_{\rho}\right), \\
j^{\mu} & =n u^{\mu}+\zeta^{(2)} \epsilon^{\mu \nu} u_{\nu} .
\end{aligned}
$$

Here $\epsilon, P, n$ refer to energy density, pressure and the charge density. $\lambda^{(2)}$ and $\zeta^{(2)}$ are the two parity odd transport coefficients which are of interest. The superscripts refer to the fact that these transport are for $d=2$. Note that we have written the constitutive relations in the anomaly frame. We work in the metric with the signature $(1,-1)$. To obtain the Kubo formula for these, consider the fluid perturbed from its rest frame $u^{\mu}=\left(1, v^{x}\right)$ with $\left|v^{x}\right| \ll 1$. We also perturb the metric and the gauge fields as follows

$$
\begin{aligned}
& g_{\mu \nu}=\eta_{\mu \nu}+h_{\mu \nu}, \quad h_{t t}, h_{t x} \neq 0, \quad h_{x x}=0, \\
& A_{t}=a_{t}, \quad A_{x}=a_{x} \text {. }
\end{aligned}
$$

Substituting this expansion in the constitutive relations in (2.1), we obtain the following expressions to the linear order

$$
\begin{aligned}
T^{t x} & =\epsilon v^{x}+P v^{x}-P h_{t x}, \\
j^{t} & =-\zeta^{(2)} v^{x}+\zeta_{v} h_{t x} .
\end{aligned}
$$

Here we have equated the first order terms in the equations for the current and called it $j^{t}$ for convenience in notation. We have also used the fact that $v_{x}=-v^{x}+h_{t x}$, to linear order. Solving for $v^{x}$ using the first equation and substituting in the constitutive relation for the current we obtain

$$
j^{t}=n-\zeta^{(2)}\left(\frac{T^{t x}+P h_{t x}}{\epsilon+P}\right)+\zeta^{(2)} h_{t x} .
$$

This can be thought of as an Ward identity obeyed by the theory. We Fourier transform the equation and then differentiate with respect to $h_{t x}$ and finally set the perturbations to zero. Using the definition

$$
\frac{1}{\sqrt{g}} \frac{\delta}{\delta g_{\mu \nu}}=-\frac{T^{\mu \nu}}{2}
$$

we obtain

$$
\left\langle j^{t}(\omega, p) T^{t x}(-\omega,-p)\right\rangle=-\zeta^{(2)}\left(\frac{\left\langle T^{t x}(\omega, p) T^{t x}(-\omega,-p)\right\rangle-P}{\epsilon+P}\right)-\zeta^{(2)} .
$$

Now we use the relation

$$
\lim _{p \rightarrow 0, \omega \rightarrow 0}\left\langle T^{t x}(\omega, p) T^{t x}(-\omega, p)\right\rangle=P,
$$

\footnotetext{
${ }^{4}$ We work in the mostly negative signature through out this paper.
} 
which can be obtained by differentiating the first equation in (2.3) with respect to $h_{t x}$ in (2.6). This leads us to the following Kubo formula

$$
-\zeta^{(2)}=\lim _{p \rightarrow 0, \omega \rightarrow 0}\left\langle j^{t}(\omega, p) T^{t x}(-\omega,-p)\right\rangle_{R}
$$

Similarly we consider the first order change in the stress tensor $T^{t t}$ from (2.1), which is given by

$$
T^{t t}=2 \lambda^{(2)}\left(-v^{x}+h_{t x}\right) .
$$

Again eliminating $v^{x}$ and differentiating the above Ward identity in Fourier space with respect to $h_{t x}$ we obtain

$$
-\lambda^{(2)}=\frac{1}{2} \lim _{p \rightarrow 0, \omega \rightarrow 0}\left\langle T^{t t}(\omega, p) T^{t x}(-\omega,-p)\right\rangle_{R} .
$$

Before proceeding we make a few observations related to the Kubo formulae for the two leading order transport coefficients in (2.8) and (2.10). Both the two point functions are obtained as the Fourier transform of the real time retarded correlator and the expectation value is taken over states held at finite temperature and chemical potential of the theory. These statements will hold true for all the correlators involving the various Kubo formulae studied in this paper. Also note that they do not involve any division by momenta, since these transport coefficients occur at the zeroth order in derivatives. We need to take the zero frequency limit first and then the zero momentum limit in (2.8) and (2.10). This is similar to the situation in higher dimensions where the static, zero frequency limit needs to be first taken and then the zero momentum limit [21]. Note that these transport coefficients in $d=2$ can also be obtained by considering one point functions of the stress tensor and the current. However we wish to illustrate the role of contact terms and also have a discussion similar to that seen in higher dimension and therefore we choose to examine two point functions. The transport coefficients of interest occur at the zeroth order in derivatives, they can in principle affect the hydrodynamic charge and sound modes ${ }^{5}$ at the zeroth order in the momentum expansions. We will study the consequences of these transport coefficients in section 5 .

\subsection{Chiral fermions}

Our goal in this section is to evaluate transport coefficients $\zeta^{(2)}$ and $\lambda^{(2)}$ given in (2.8) and (2.10) for a theory of free chiral fermions held at finite temperature and finite chemical potential. As we have discussed earlier, it is the Fourier transform of the real time retarded correlators which determine the transport coefficients. We obtain these correlators by first evaluating them in the Euclidean theory and then performing the necessary analytic continuation. Consider the Euclidean partition function $\mathcal{S}_{E}$ of free chiral fermions, coupled to background metric and gauge field. The subscript $E$ will always refer to Euclidean. It admits the following expansion around the unperturbed background given in (2.2)

$$
S_{E}=S_{E}^{(0)}+\frac{1}{\sqrt{g}} \frac{\delta S_{E}}{\delta A_{\mu}} A_{\mu}+\frac{1}{\sqrt{g}} \frac{\delta S_{E}}{\delta g_{\mu \nu}} g_{\mu \nu}+\frac{1}{2 \sqrt{g}} \frac{\delta}{\delta g_{\rho \sigma}}\left(\frac{1}{\sqrt{g}} \frac{\delta S_{E}}{\delta g_{\mu \nu}}\right) g_{\mu \nu} g_{\rho \sigma}
$$

\footnotetext{
${ }^{5}$ For relativistic fluids in $1+1$ dimensions, these hydrodynamic modes were studied in [24].
} 


$$
+\frac{1}{2 \sqrt{g}} \frac{\delta}{\delta g_{\rho \sigma}}\left(\frac{1}{\sqrt{g}} \frac{\delta S_{E}}{\delta A_{\mu}}\right) A_{\mu} g_{\rho \sigma}+\cdots
$$

Note that due to minimal coupling, the gauge field $A_{\mu}$ occurs at most with a single power in the above expansion. The partition function of the theory is given by

$$
Z_{E}=\int \mathcal{D} \psi^{\dagger} \mathcal{D} \psi \exp \left(S_{E}\right)
$$

Here $\psi$ refers to the Fermion. Then the expectation value of the current and the stress tensor are defined by

$$
\begin{aligned}
\left\langle j^{\mu}\right\rangle_{E} & =-\left.\frac{1}{\sqrt{g}} \frac{\delta \ln \mathcal{Z}_{E}}{\delta A_{\mu}}\right|_{a_{\mu}, h_{\mu \nu}=0}=-\left.\frac{1}{\mathcal{Z}_{E}^{(0)}} \int \mathcal{D} \psi^{\dagger} \mathcal{D} \psi e^{S_{E}^{(0)}} \frac{1}{\sqrt{g}} \frac{\delta S_{E}}{\delta A_{\mu}}\right|_{a_{\mu}, h_{\mu \nu}=0} \\
\left\langle T^{\mu \nu}\right\rangle_{E} & =-\left.2 \frac{1}{\sqrt{g}} \frac{\delta \ln \mathcal{Z}_{E}}{\delta g_{\mu \nu}}\right|_{a_{\mu}, h_{\mu \nu}=0}=-\left.\frac{2}{\mathcal{Z}_{E}^{(0)}} \int \mathcal{D} \psi^{\dagger} \mathcal{D} \psi e^{S_{E}^{(0)}} \frac{1}{\sqrt{g}} \frac{\delta S_{E}}{\delta g_{\mu \nu}} g_{\mu \nu}\right|_{a_{\mu}, h_{\mu \nu}=0}
\end{aligned}
$$

where $\mathcal{Z}^{(0)}$ refers to the partition function in the absence of any perturbation. To unclutter notations, from now all derivatives in the fields are understood to be evaluated at $a_{\mu}, h_{\mu \nu}=0$. The relevant two point functions in the Euclidean theory are given by

$$
\left\langle j^{\mu} T^{\rho \sigma}\right\rangle_{E}=2 \frac{1}{\sqrt{g}} \frac{\delta}{\delta g_{\rho \sigma}}\left(\frac{1}{\sqrt{g}} \frac{\delta \ln \mathcal{Z}_{E}}{\delta A_{\mu}}\right) .
$$

Substituting the expansion of the action given in (2.11) into the definition of the partition function we obtain

$$
\begin{aligned}
\left\langle j^{\mu} T^{\rho \sigma}\right\rangle_{E}= & -\left\langle j^{\mu}\right\rangle_{E}\left\langle T^{\rho \sigma}\right\rangle_{E}+\frac{2}{\mathcal{Z}_{E}^{(0)}} \int \mathcal{D} \psi^{\dagger} \mathcal{D} \psi e^{S_{E}^{(0)}} \frac{1}{\sqrt{g}} \frac{\delta S_{E}}{\delta A_{\mu}} \frac{1}{\sqrt{g}} \frac{\delta S_{E}}{\delta g_{\rho \sigma}} \\
& +\frac{2}{\mathcal{Z}_{E}^{(0)}} \int \mathcal{D} \psi^{\dagger} \mathcal{D} \psi e^{S_{E}^{(0)}} \frac{1}{\sqrt{g}} \frac{\delta}{\delta g_{\rho \sigma}}\left(\frac{1}{\sqrt{g}} \frac{\delta S_{E}}{\delta A_{\mu}}\right) .
\end{aligned}
$$

Note the disconnected contribution of the first term cancels the disconnected contributions from the second term, in the first line of the above equation. The non-zero contribution of the correlator arises from the connected contribution of the second term and the from the last term. Since $S_{E}$ is a bilinear in the fermions, the third term is a contact term. We will see that such terms contribute crucially to the correlator and it is only after adding these terms, the 'replacement rule' for chiral fermions can be demonstrated. The structure of these terms in $d=2$ is different from that in higher dimensions. On performing the same manipulations for correlator corresponding to $\lambda^{(2)}$, we obtain

$$
\begin{aligned}
\left\langle T^{\mu \nu} T^{\rho \sigma}\right\rangle_{E}= & \frac{1}{\sqrt{g}} \frac{\delta}{\delta g_{\rho \sigma}}\left(\frac{1}{\sqrt{g}} \frac{\delta \ln \mathcal{Z}_{E}}{\delta g_{\mu \nu}}\right) \\
= & -\left\langle T^{\mu \nu}\right\rangle_{E}\left\langle T^{\rho \sigma}\right\rangle_{E}+\frac{4}{\mathcal{Z}_{E}^{(0)}} \int \mathcal{D} \psi^{\dagger} \mathcal{D} \psi e^{S_{E}^{(0)}} \frac{1}{\sqrt{g}} \frac{\delta S_{E}}{\delta g_{\mu \nu}} \frac{1}{\sqrt{g}} \frac{\delta S_{E}}{\delta g_{\rho \sigma}} \\
& +\frac{4}{\mathcal{Z}_{E}^{(0)}} \int \mathcal{D} \psi^{\dagger} \mathcal{D} \psi e^{S_{E}^{(0)}} \frac{1}{\sqrt{g}} \frac{\delta}{\delta g_{\rho \sigma}}\left(\frac{1}{\sqrt{g}} \frac{\delta S_{E}}{\delta g_{\mu \nu}}\right) .
\end{aligned}
$$


Here again the disconnected terms cancel and we need to examine only the connected diagrams to evaluate the above correlator.

After evaluating the Euclidean two point functions, we perform the following analytic continuation to obtain the retarded correlator

$$
\left\langle j^{\tau}\left(\omega_{n}, p\right) T^{\tau x}\left(-\omega_{n},-p\right)\right\rangle_{E}=-\left.\left\langle j^{t}(\omega, p) T^{t x}(-\omega,-p)\right\rangle_{R}\right|_{i \omega_{n} \rightarrow p_{0}+i \epsilon} .
$$

Here $\omega_{n}=2 \pi n T$, where $T$ is the temperature and $n \in Z$ refer to the Matsubara frequencies. Note that all external Matsubara frequencies are even multiples of $\pi T$ and will be labelled by $n$. The overall negative sign results from converting the tensor indices containing the Euclidean time $\tau$ to Minkowski time $t$ using the replacement $\tau \rightarrow i t$. Similarly we have

$$
\left\langle T^{\tau \tau}\left(\omega_{n}, p\right) T^{\tau x}\left(-\omega_{n},-p\right)\right\rangle_{E}=-\left.i\left\langle T^{t t}(\omega, p) T^{t x}(-\omega,-p)\right\rangle_{R}\right|_{i \omega_{n} \rightarrow p_{0}+i \epsilon}
$$

Finally to obtain the transport coefficients, we use the Kubo formula in (2.8) and (2.10).

Let us now proceed to implement the above procedure in detail. The first step is to write down the expansion of the Euclidean action of chiral fermions in background gauge field and metric. The action is given by

$$
S_{E}=\int d \tau d x \sqrt{g} e_{a}^{\mu} \psi^{\dagger} \gamma^{a} D_{\mu} P_{-} \psi
$$

The gamma matrices convention, we choose to work with, are given by

$$
\gamma^{\tau}=i \sigma^{1}, \quad \gamma^{x}=i \sigma^{2}, \quad \gamma_{c}=-i \gamma^{\tau} \gamma^{x}=-\sigma^{3} .
$$

The chiral projection operators are defined by

$$
P_{\mp}=\frac{1}{2}\left(1 \mp \gamma_{c}\right) \text {. }
$$

Note that the Euclidean gamma matrices are anti-Hermitian, $\gamma_{c}$ is Hermitian and $P_{-}$ projects on to the upper component of spinor, while $P_{+}$retains the lower component of the spinor. The gamma matrices obey the algebra

$$
\left\{\gamma^{a}, \gamma^{b}\right\}=2 \eta_{E}^{a b}, \quad \eta_{E}^{a b}=-\delta^{a b},
$$

$e_{a}^{\mu}$ is the vierbien and the covariant derivative $D_{\mu}$ is defined by

$$
D_{\mu}=\partial_{\mu}+\frac{1}{2} \omega_{\mu c d} \sigma^{c d}+i A_{\mu}, \quad \sigma^{c d}=\frac{i}{4}\left[\gamma^{c} \gamma^{d}\right]
$$

In two dimensions, the term involving the spin connection $\omega_{\mu c d}$ vanishes and we do not consider it for the rest of this section. We will see in the subsequent sections that the contact terms in higher dimensions arise from linearizing the spin connection. In $d=2$ the source of the contact terms arise from the linearization of the metric and the vierbein.

We proceed to expand the action (2.19) in terms of the following perturbations in the metric and the gauge field

$$
g_{\tau \tau}=-1+h_{\tau \tau}, \quad g_{\tau x}=g_{x \tau}=h_{\tau x}, \quad g_{x x}=-1,
$$




$$
A_{\tau}=A_{\tau}^{(0)}+a_{\tau}, \quad A_{x}=0 .
$$

Here $A_{\tau}^{(0)}$ refers to the constant background chemical potential, which will be turned on subsequently. The inverse metric, to the second order in the perturbations is given by

$$
g^{\mu \nu}=\left(\begin{array}{ll}
-1-h_{\tau \tau}-h_{\tau \tau}^{2}-h_{\tau x}^{2} & -h_{\tau x}-h_{\tau \tau} h_{\tau x} \\
-h_{\tau x}-h_{\tau \tau} h_{\tau x} & -1-h_{\tau x}^{2}
\end{array}\right)+O\left(h^{3}\right) .
$$

Using the gauge in [23] the vierbein is given by

$$
e_{a \mu}=\left(\begin{array}{cc}
-1+\frac{h_{\tau \tau}}{2}+\frac{1}{8}\left(h_{\tau x}^{2}+h_{\tau \tau}^{2}\right) & \frac{h_{\tau x}}{2}+\frac{h_{\tau \tau} h_{\tau x}}{8} \\
\frac{h_{\tau x}}{2}+\frac{h_{\tau \tau} h_{\tau x}}{8} & -1+\frac{h_{\tau x}^{2}}{8}
\end{array}\right)+O\left(h^{3}\right) .
$$

Finally the inverse vierbein is given by

$$
\begin{array}{rlrl}
e_{\hat{\tau}}^{\tau} & =1+\frac{h_{\tau \tau}}{2}+\frac{3}{8}\left(h_{\tau x}^{2}+h_{\tau \tau}^{2}\right)+O\left(h^{3}\right), & & e_{\hat{x}}^{\tau}=\frac{h_{\tau x}}{2}+\frac{3}{8}\left(h_{\tau x} h_{\tau \tau}\right)+O\left(h^{3}\right) \\
e_{\hat{\tau}}^{x}=\frac{h_{\tau x}}{2}+\frac{3}{8}\left(h_{\tau x} h_{\tau \tau}\right)+O\left(h^{3}\right), & e_{\hat{x}}^{x}=1+\frac{3}{8} h_{\tau x}^{2}+O\left(h^{3}\right) .
\end{array}
$$

where the hatted variables refer to the flat space co-ordinates. We now substitute these expansions in (2.19) and obtain the following action, to the leading orders in the perturbations.

$$
\begin{aligned}
S_{E}= & S_{E}^{(1)}+S_{E}^{(2)} \\
S_{E}^{(1)}= & \int d^{2} x \sqrt{g}\left[\psi^{\dagger}(x) \gamma^{\tau} D_{\tau}^{(0)} P_{-} \psi(x)+\psi^{\dagger}(x) \gamma^{x} \partial_{x} P_{-} \psi(x)\right] \\
& -\frac{h_{\tau \tau}}{2} \psi^{\dagger}(x) \gamma^{\tau} D^{\tau(0)} P_{-} \psi(x)-\frac{h_{\tau x}}{2} \psi^{\dagger}(x)\left(\gamma^{x} D^{\tau(0)}+\gamma^{\tau} \partial^{x}\right) P_{-} \psi(x) \\
& \times\left[-\frac{3 h_{\tau \tau} h_{\tau x}}{8}\left(\psi^{\dagger}(x) \gamma^{x} D^{\tau(0)} P_{-} \psi(x)+\psi^{\dagger}(x) \gamma^{\tau} \partial^{x} P_{-} \psi(x)\right)\right]+\cdots \\
S_{E}^{(2)}= & i e \int d^{2} x \sqrt{g}\left[\left(a_{\tau} \psi^{\dagger}(x) \gamma^{\tau} P_{-} \psi(x)+\frac{h_{\tau x}}{2} a_{\tau} \psi^{\dagger}(x) \gamma^{x} P_{-} \psi(x)\right]+\cdots\right.
\end{aligned}
$$

where

$$
D_{\tau}^{(0)}=\partial_{\tau}+i e A_{\tau}^{(0)},
$$

is the covariant derivative in the background chemical potential. In (2.28) and (2.28), we have retained only the terms relevant to obtain the correlators of interest. Note also, we have used the on-shell condition to simplify the coefficient of $h_{\tau \tau}$. The components of the flat space stress tensor of interest is then given by

$$
T_{f l}^{\tau \tau}(x)=\psi^{\dagger} \gamma^{\tau} D^{\tau(0)} P_{-} \psi, \quad T_{f l}^{\tau x}(x)=\frac{1}{2} \psi^{\dagger}\left(\gamma^{\tau} \partial^{x}+\gamma^{x}\left(D^{\tau(0)}\right) P_{-} \psi .\right.
$$

Similarly the time component of the flat space charge current is given by

$$
j_{f l}^{\tau}=-i e \psi^{\dagger} \gamma^{\tau} P_{-} \psi
$$


Note that the quadratic terms involving the perturbations in (2.28) and (2.29) are responsible for contact terms in the two point functions of interest. These terms contribute in the correlators for $d=2$ because the one needs only 2 gamma matrices to obtain a nonzero trace along with $\gamma_{c}$. As a simple cross check on these terms we considered the stress tensor in curved space and expanded it to first order in metric perturbations. The first derivative of the curved space stress tensor agrees with the second derivative of the action with respective to the metric obtained from 2.28 and 2.29 .

The last ingredient we require to perform the computation of the two point function, is the propagator for the free chiral fermions. From the first term in the action given in (2.28), the Euclidean propagator at finite temperature and finite chemical potential in momentum space is given by

$$
\left\langle\psi\left(\omega_{m}, p\right) \psi^{\dagger}\left(\omega_{m^{\prime}}, p^{\prime}\right)\right\rangle=S\left(\omega_{m}, p\right) \delta_{m, m^{\prime}} \delta\left(p-p^{\prime}\right) 2 \pi,
$$

and

$$
\begin{aligned}
S\left(\omega_{m}, p\right) & =-i\left[\gamma^{\tau}\left(\omega_{m}-e i \mu-e i \mu_{5} \gamma_{c}\right)+\gamma^{x} p\right]^{-1} \\
& =\left(\begin{array}{cc}
0 & \frac{-i}{i \omega_{m}+e\left(\mu-\mu_{c}\right)-p} \\
\frac{-i}{i \omega_{m}+e\left(\mu+\mu_{c}\right)+p} & 0
\end{array}\right) .
\end{aligned}
$$

Here $\omega_{m}=(2 m+1) \pi T$ is the Fermionic Matsubara frequency at temperature $T$. The label $m$ will refer to Matsubara frequencies which occur as odd multiples of $\pi T$. We have chosen the background chemical potential to be

$$
A_{\tau}^{(0)}=i \mu+i \mu_{c} \gamma_{c}
$$

where $\mu$ is the chemical potential corresponding to the vector $\mathrm{U}(1)$ and $\mu_{c}$ is the chemical potential corresponding to the axial U(1) current. ${ }^{6}$ Note that the transformation to the momentum space is given by

$$
\begin{aligned}
\psi(x) & =\frac{1}{\beta} \sum_{\omega_{m}} \int \frac{d p}{2 \pi} \psi(p) e^{-i\left(\omega_{m} \tau+p x\right)}, \\
\psi^{\dagger}(x) & =\frac{1}{\beta} \sum_{\omega_{m}} \int \frac{d p}{2 \pi} \psi^{\dagger}(p) e^{i\left(\omega_{m} \tau+p x\right)} .
\end{aligned}
$$

This explains the relative sign between the frequency and the chemical potential in the propagator.

$\boldsymbol{\zeta}^{(2)}$ from chiral fermions. Let us now evaluate the Euclidean correlator $\left\langle j^{\tau} T^{\tau x}\right\rangle$. From (2.15) and (2.29), we see that there are two contributions. They are given by

$$
\begin{aligned}
& \left\langle j^{\tau}(p) T^{\tau x}(-p)\right\rangle=A+B, \\
& A=\left\langle j_{f l}^{\tau}(p) T_{f l}^{\tau x}(-p)\right\rangle_{c}, \quad B=\frac{i e}{2} \int \frac{d^{2} p^{\prime}}{(2 \pi)^{2}}\left\langle\psi^{\dagger}\left(p^{\prime}\right) \gamma^{x} P_{-} \psi\left(p^{\prime}\right)\right\rangle .
\end{aligned}
$$

\footnotetext{
${ }^{6}$ The signs for the chemical potentials are fixed using the thermodynamic definition $\frac{\partial \ln \mathcal{Z}}{\partial \mu}=j^{0}$. This allows us to identify the Euclidean gauge field to be $A_{\tau}^{E}=i \mu$.
} 


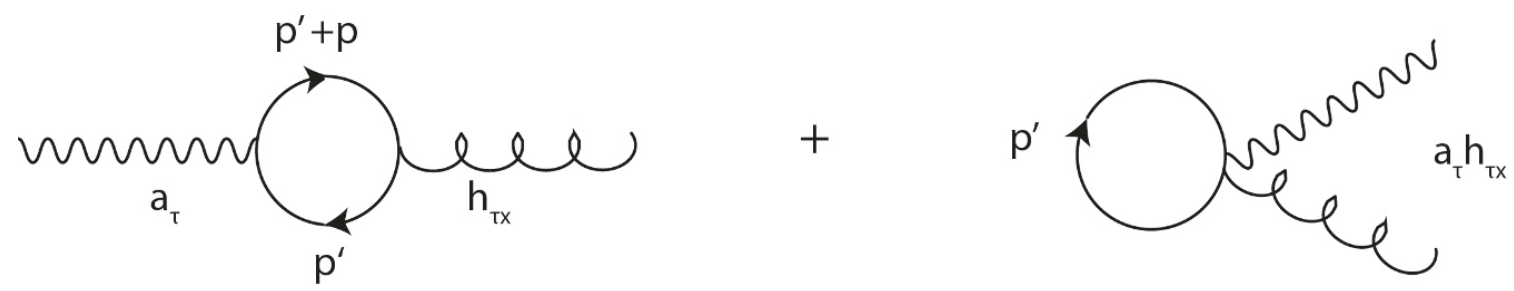

Figure 1. Diagrams contributing to $\zeta^{(2)}$.

Here, for convenience of notation, we have used $p$ to refer to $\left(p_{0}, p_{x}\right)=\left(\omega_{m}, p_{x}\right)$ where $\omega_{m}$ is the corresponding Matsubara frequency. The integral in the second term $B$ stands for

$$
\int \frac{d^{2} p}{(2 \pi)^{2}} \rightarrow \frac{1}{\beta} \sum_{n} \int \frac{d p_{x}}{2 \pi}
$$

Note that the term $B$ arises from the second contribution in (2.15). The Fourier transform of the stress tensor and the current, in flat space, are given by

$$
\begin{aligned}
j_{f l}^{\tau}(p) & =-i e \int \frac{d^{2} p^{\prime}}{(2 \pi)^{2}} \psi^{\dagger}\left(p^{\prime}-p\right) \gamma^{\tau} P_{-} \psi\left(p^{\prime}\right), \\
T_{f l}^{\tau x}(-p) & =\frac{1}{2} \int \frac{d^{2} p^{\prime}}{(2 \pi)^{2}} \psi^{\dagger}\left(p^{\prime}+p\right)\left[\gamma^{\tau} i p_{x}^{\prime}+\gamma^{x}\left(i p_{0}^{\prime}+e\left(\mu+\mu_{c} \gamma_{c}\right)\right)\right] P_{-} \psi\left(p^{\prime}\right) .
\end{aligned}
$$

Substituting these expressions in (2.37) and performing the necessary Wick contractions, we obtain for term $A$

$$
\begin{aligned}
A & =\frac{i e}{2} \int \frac{d^{2} p^{\prime}}{(2 \pi)^{2}} \operatorname{Tr}\left[\gamma^{\tau} S\left(p^{\prime}+p\right)\left(\gamma^{\tau} i p_{x}^{\prime}+\gamma^{x}\left(i p_{0}^{\prime}+e\left(\mu+\mu_{c} \gamma_{c}\right)\right)\right) S\left(p^{\prime}\right) P_{+}\right] \\
& =-\sum_{m} \frac{e}{2 \beta} \int \frac{d p_{x}^{\prime}}{2 \pi}\left(\frac{p_{x}^{\prime}+i \omega_{m}+e \mu_{-}}{\left(i \omega_{m}+i \omega_{n}+e \mu_{-}-\left(p_{x}^{\prime}+p_{x}\right)\right)\left(i \omega_{m}+e \mu_{-}-p_{x}^{\prime}\right)}\right)
\end{aligned}
$$

where

$$
\mu_{-}=\mu-\mu_{c}
$$

Note that there is an over all $2 \pi \beta \delta(0)$ resulting from momentum conservation, which we have factored out. We perform the sum over Matsubara Fermionic frequencies by the usual trick of converting the sum to a contour integral. Here are the results for the sum

$$
\begin{gathered}
\left.\sum_{m} \frac{1}{\beta}\left[\left(i \omega_{m}+e \mu_{-}-p_{x}^{\prime}\right)\right)\left(i \omega_{m}+e \mu_{-}+i \omega_{n}-p_{x}^{\prime}-p_{x}\right)\right]^{-1} \\
=\frac{f\left(p_{x}^{\prime}-e \mu_{-}\right)-f\left(p_{x}^{\prime}+p_{x}-\mu_{-}-i \omega_{n}\right)}{i \omega_{n}-p_{x}}, \\
\sum_{m} \frac{1}{\beta} \frac{i \omega_{m}+e \mu_{-}}{\left(i \omega_{m}+e \mu_{-}-p_{x}^{\prime}\right)\left(i \omega_{m}+e \mu_{-}+i \omega_{n}-p_{x}^{\prime}-p_{x}\right)} \\
=\frac{p_{x}^{\prime} f\left(p_{x}^{\prime}-e \mu_{-}\right)-\left(p_{x}+p_{x}^{\prime}-i \omega_{n}\right) f\left(p_{x}+p_{x}^{\prime}-i \omega_{n}\right)}{i \omega_{n}-p_{x}}-\frac{1}{2},
\end{gathered}
$$


and $f$ is the Fermi-Dirac distribution given by

$$
f(x)=\frac{1}{1+e^{\beta x}} .
$$

Note that the temperature, chemical potential independent constant $1 / 2$ in the second sum can be ignored, since it just results in infinite constant. Substituting the results for these sums into the expression for $A$ in (2.40), we obtain

$$
A=\frac{e}{2} \int_{-\infty}^{\infty} \frac{d p_{x}^{\prime}}{2 \pi}\left(\frac{2 p_{x}^{\prime} f\left(p_{x}^{\prime}-e \mu_{-}\right)}{p_{x}-i \omega_{n}}-\frac{\left(p_{x}+2 p_{x}^{\prime}-i \omega_{n}\right) f\left(p_{x}+p_{x}^{\prime}-i \omega_{n}\right)}{p_{x}-i \omega_{n}}\right)
$$

We can now change variables in the integration for the 2 nd term and combine both the terms as ${ }^{7}$

$$
A=\frac{e}{2} \frac{p_{x}+i \omega_{n}}{p_{x}-i \omega_{n}} \int \frac{d p_{x}^{\prime}}{2 \pi} f\left(p_{x}^{\prime}-e \mu_{-}\right) .
$$

Let us now examine the term $B$, again performing the required Wick contraction we obtain

$$
\begin{aligned}
B & =-i \frac{e}{2} \int \frac{d^{2} p^{\prime}}{(2 \pi)^{2}} \operatorname{Tr}\left(\gamma^{x} S\left(p^{\prime}\right) P_{+}\right), \\
& =\frac{e}{2 \beta} \sum_{m} \int \frac{d p_{x}^{\prime}}{2 \pi} \frac{1}{i \omega_{m}+e \mu_{-}-p_{x}^{\prime}} .
\end{aligned}
$$

After performing the Matsubara sum we obtain

$$
B=\frac{e}{2} \int_{-\infty}^{\infty} \frac{d p_{x}^{\prime}}{2 \pi} f\left(p_{x}^{\prime}-e \mu_{-}\right)
$$

Adding the contributions in (2.45) and (2.47) the $\left\langle j^{\tau} T^{\tau x}\right\rangle$ correlator is given by

$$
\begin{aligned}
\left\langle j^{\tau}(p) T^{\tau x}(-p)\right\rangle & =e \frac{p_{x}}{p_{x}-i \omega_{n}} \int_{-\infty}^{\infty} d p_{x}^{\prime} f\left(p_{x}^{\prime}-e \mu_{-}\right), \\
& =e \frac{p_{x}}{p_{x}-i \omega_{n}} \int_{0}^{\infty} \frac{d p_{x}^{\prime}}{2 \pi}\left(f\left(p_{x}^{\prime}-e \mu_{-}\right)-f\left(p_{x}^{\prime}+e \mu_{-}\right)\right) \\
& =\frac{p_{x}}{p_{x}-i \omega_{n}} \frac{e^{2} \mu_{-}}{2 \pi} .
\end{aligned}
$$

To obtain the second line of the above equation, we have again ignored an infinite constant, which is independent of temperature and chemical potential. The last line is obtained using the results of appendix A, equation (A.2), to perform the integral over the Fermi-Dirac distribution. We can obtain the retarded correlator using (2.17)

$$
\left\langle j^{t}\left(p_{0}, p_{x}\right) T^{t x}\left(-p_{0},-p_{x}\right)\right\rangle_{R}=-\frac{p_{x}}{p_{x}-p_{0}} \frac{e^{2} \mu_{-}}{2 \pi} .
$$

Here $\left(p_{0}, p_{x}\right)$ refer to the frequency and momentum in Minkowski space. We have ignored the $i \epsilon$ that results from using (2.17), which is required when one needs to transform this

\footnotetext{
${ }^{7}$ Though there is a divergence in the integrals when $p_{x}^{\prime} \rightarrow-\infty$ it can be shown that this infinite constant is independent of temperature or chemical potentials. The change of variables can be justified on careful treatment of the integrals when $p_{x}^{\prime}<0$.
} 
correlator in Fourier space to a real time correlator. Finally using (2.8), the result for the transport coefficient is obtained by first taking the zero frequency limit $p_{0} \rightarrow 0$ and then the zero momentum limit $p_{x} \rightarrow 0$. This results in

$$
\begin{aligned}
\zeta^{(2)} & =-\lim _{p_{x} \rightarrow 0, p_{0} \rightarrow 0}\left\langle j^{t}\left(p_{0}, p_{x}\right) T^{t x}\left(-p_{0},-p_{x}\right)\right\rangle_{R}, \\
& =\frac{e^{2} \mu_{-}}{2 \pi} .
\end{aligned}
$$

Examining (2.49), we see that it was crucial to take the zero frequency limit first and then the zero momentum limit. Note that both term $A$ and the 'contact' term $B$ in (2.37) contribute and they contribute equally in the zero frequency and zero momentum limit. Also note that in $d=2$ term $B$ contributed since one needed only 2 gamma matrices to saturate the trace. If once considers the contribution to this transport coefficient from the anti-chiral Fermions with chemical potential, then through a similar analysis one obtains

$$
\zeta_{\text {anti-chiral }}^{(2)}=-\frac{e^{2} \mu_{+}}{2 \pi},
$$

where

$$
\mu_{+}=\mu+\mu_{c}
$$

From (2.50) and (2.51), we see that the transport coefficient vanishes for a Dirac fermion in the absence of the chiral chemical potential $\mu_{c}$.

Let us now compare the transport coefficient for chiral fermions in (2.50), with the coefficient of the $\mathrm{U}(1)$ anomaly. The anomaly equation satisfied by the chiral current $j^{\mu}=e \bar{\psi} \gamma^{\mu} P_{-} \psi$, is given by

$$
\partial_{\mu} j^{\mu}=c_{s} \epsilon^{\mu \nu} F_{\mu \nu}, \quad c_{s}=-\frac{e^{2}}{4 \pi} .
$$

The value of $c_{s}$ for a Weyl Fermion in 2 dimensions can be read out at many places in the literature, see for instance equation (2.27) of [12]. We now compare the coefficient of the anomaly and the transport coefficient to see that they are related by

$$
\zeta^{(2)}=-2 c_{s} \mu_{-}
$$

which is the 'replacement rule' obtained in $[7,14,15]$. Observe that we have arrived at this relation between the transport coefficient and the microscopic anomaly from explicitly evaluating the diagrams that contribute to the Kubo formula. Note that the 'contact' terms gave rise to an equal contribution.

For later purpose it is useful to parametrize the anomaly coefficients with the help of the anomaly polynomial. The anomalies of a theory in $d$ dimensions, are encoded in a $d+2$ form constructed out of gauge field strengths $\hat{F}$ and curvature tensor $\hat{R}_{a b}$. We define the curvature two-form $\hat{R}_{a b}$ and the field strength two-form as

$$
\hat{R}_{a b}=\frac{1}{2} R_{a b c d} d x^{c} \wedge d x^{d}, \quad \hat{F}=\frac{1}{2} F_{a b} d x^{a} \wedge d x^{b} .
$$




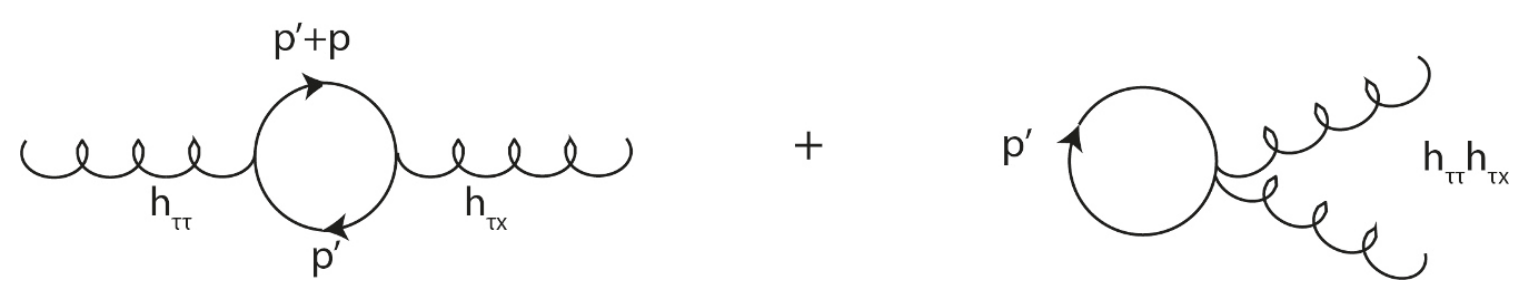

Figure 2. Diagrams contributing to $\lambda^{(2)}$.

Furthermore, let us define a $2 k$-form which is a $k$-th degree polynomial in the curvature 2 -forms

$$
\hat{R}_{k} \equiv \frac{1}{2} \hat{R}_{a_{2}}^{a_{1}} \wedge \hat{R}_{a_{3}}^{a_{2}} \wedge \cdots \hat{R}_{a_{1}}^{a_{k}} .
$$

The anomaly polynomial for a single Weyl fermion in $d=2$ takes the form [7]

$$
\mathcal{P}_{d=1+1}(\hat{F}, \hat{R})=c_{s} F \wedge F+c_{g} \operatorname{tr}(\hat{R} \wedge \hat{R})
$$

where,

$$
c_{s}=-\frac{e^{2}}{4 \pi}, \quad c_{g}=-\frac{1}{96 \pi} .
$$

$\lambda^{(2)}$ from chiral fermions. We evaluate the correlator $\left\langle T^{\tau \tau} T^{\tau x}\right\rangle$. Again from (2.16) and (2.28), we see that this correlator receives contributions from two terms which are given by

$$
\begin{aligned}
& \left\langle T^{\tau \tau}(p) T^{\tau x}(-p)\right\rangle=A+B, \\
& A=\left\langle T_{f l}^{\tau \tau}(p) T_{f l}^{\tau x}(-p)\right\rangle_{c}, \quad B=-\frac{6}{8}\left\langle\int \frac{d^{2} p}{(2 \pi)^{2}} \psi^{\dagger}\left[\gamma^{x}\left(i p_{\tau}+\left(\mu+\mu_{c} \gamma_{c}\right)\right)+\gamma^{\tau} i p_{x}\right] P_{-} \psi\right\rangle .
\end{aligned}
$$

To evaluate the term $A$ we need

$$
T_{f l}^{\tau \tau}(p)=\int \frac{d^{2} p^{\prime}}{(2 \pi)^{2}} \psi^{\dagger}\left(p^{\prime}-p\right) \gamma^{\tau}\left(i p_{\tau}+e\left(\mu+\mu_{c} \gamma_{c}\right)\right) P_{-} \psi(p) .
$$

Inserting this and the expression for $T^{\tau x}(-p)$ from (2.39), and performing the Wick contractions using the propagator in $(2.34)$, we obtain

$$
\begin{aligned}
A\left(\omega_{n}, p_{x}\right)=-\frac{1}{2 \beta} & \sum_{m} \int \frac{d p^{\prime}}{2 \pi} \operatorname{Tr}\left(\gamma^{\tau}\left[i\left(\omega_{n}+\omega_{m}\right)+e\left(\mu+\mu_{c} \gamma_{c}\right)\right]\right. \\
& \times S\left(\omega_{n}+\omega_{m}, p_{x}+p_{x}^{\prime}\right)\left[i \gamma^{\tau} p_{x}^{\prime}+\gamma^{x}\left(i \omega_{m}+e\left(\mu+\mu_{c} \gamma_{c}\right)\right] S\left(\omega_{m}, p_{x}^{\prime}\right) P_{+}\right) .
\end{aligned}
$$

Substituting the expression for the propagator, performing the trace and the Matsubara sum results in

$$
\begin{aligned}
\lim _{p_{x} \rightarrow 0} A\left(0, p_{x}\right) & =\frac{i}{2} \int_{0}^{\infty} \frac{d p_{x}^{\prime}}{2 \pi} p_{x}^{\prime}\left(f\left(p_{x}^{\prime}-e \mu_{-}\right)+f\left(p_{x}^{\prime}+e \mu_{-}\right)\right), \\
& =\frac{i}{8 \pi}\left(e^{2} \mu_{-}^{2}+\frac{\pi^{2} T^{2}}{3}\right) .
\end{aligned}
$$


In the last line we have used the identity (A.4) to perform the integral. Evaluating $B$ using the propagator given in (2.34), we obtain

$$
\begin{aligned}
B & =\frac{6}{8 \beta} \sum_{n} \int \frac{d p_{x}}{2 \pi} \operatorname{Tr}\left[\gamma^{x}\left(i \omega_{n}+\left(\mu+\mu_{c} \gamma_{c}\right)\right)+\gamma^{\tau} i p_{x} S\left(\omega_{n}, p_{x}\right)\right] \\
& =\frac{3 i}{2} \int_{0}^{\infty} \frac{d p_{x}}{2 \pi} p_{x}\left(f\left(p_{x}-e \mu_{-}\right)+f\left(p_{x}+e \mu_{-}\right)\right) \\
& =\frac{3 i}{8 \pi}\left(e^{2} \mu_{-}^{2}+\frac{\pi^{2} T^{2}}{3}\right) .
\end{aligned}
$$

In the second line we have performed the resulting Matsubara sum, after which we have used (A.4) to perform the resulting integral. Finally adding the contributions from terms $A$ and $B$ results in

$$
\lim _{p_{x} \rightarrow 0}\left\langle T^{\tau \tau}\left(0, p_{x}\right) T^{\tau x}\left(0,-p_{x}\right)\right\rangle=\frac{i}{2 \pi}\left(e^{2} \mu_{-}^{2}+\frac{\pi^{2} T^{2}}{3}\right) .
$$

Using (2.18), the retarded correlator in Minkowski space is given by

$$
\lim _{p_{x} \rightarrow 0}\left\langle T^{t t}\left(0, p_{x}\right) T^{t x}\left(0,-p_{x}\right)\right\rangle=-\frac{1}{2 \pi}\left(e^{2} \mu_{-}^{2}+\frac{\pi^{2} T^{2}}{3}\right) .
$$

Substituting this result in the expression (2.10), the transport coefficient of interest is given by

$$
\lambda^{(2)}=\frac{1}{4 \pi}\left(e^{2} \mu_{-}^{2}+\frac{\pi^{2} T^{2}}{3}\right) .
$$

We now compare this transport coefficient with the coefficient of the gravitational anomaly of the Weyl Fermion, defined by the conservation law

$$
\nabla_{\mu} T^{\mu \nu}=F_{\nu}^{\mu} J^{\nu}+c_{g} \epsilon^{\mu \nu} \nabla_{\nu} R, \quad c_{g}=-\frac{1}{96 \pi} .
$$

The coefficient $c_{g}$ for a Weyl Fermion is obtained from (2.57). In [12], the transport coefficient $\lambda^{(2)}$ was parametrized as

$$
\lambda^{(2)}=\tilde{c}_{2 d} T^{2}-c_{s} \mu_{-}^{2} .
$$

Comparing with the result from evaluation of the Kubo formula given in (2.66), we see

$$
c_{s}=-\frac{e^{2}}{4 \pi}, \quad \tilde{c}_{2 d}=\frac{\pi}{12} .
$$

The value for $c_{s}$ agrees with the chiral anomaly of a Weyl fermion in 2 dimensions as in (2.53). The value for $\tilde{c}_{2 d}$ obeys the relation

$$
\tilde{c}_{2 d}=-8 \pi^{2} c_{g} .
$$

as argued in [12] using the consistency of the Euclidean vacuum. We have arrived at these relations by explicitly evaluating the diagrams which contribute to the Kubo Formula. Note that for both transport coefficients, there was a contribution from the 'contact' term which was important in obtaining these relations. 


\subsection{Chiral bosons}

In 2 dimensions a massless chiral Weyl fermion is dual to a chiral boson. Therefore we expect the contributions of a chiral boson to the transport coefficient $\zeta^{(2)}$ and $\lambda^{(2)}$, to be identical to that of the chiral fermions studied in section 2.1. In this section we will show that it is indeed the case. Though the result is expected on general grounds, performing the calculation in detail will serve as a cross check of the analysis in section 2.1. Also as far as we are aware transport coefficients have never been evaluated directly for chiral bosons. With these motivations we proceed with the analysis of transport in this system.

Chiral bosons in the Euclidean theory obey the constraint ${ }^{8}$

$$
\partial^{\mu} \phi=i \frac{\epsilon^{\mu \nu}}{\sqrt{g}} \partial_{\nu} \phi
$$

Note that this is the constraint which in flat space reduces to $\partial_{\bar{z}} \phi=0$ on using the change of variables to holomorphic and anti-holomorphic coordinates given in (2.109). The constraint ensures that the bosons depend only $z$, this property is is identical to the Weyl fermions considered in section 2.1. To write down the stress tensor and the current of the chiral boson theory, we impose the constraint of (2.71) in the stress tensor of the boson and the current following [23]. They are given by

$$
\begin{aligned}
T^{\mu \nu} & =\frac{-1}{8}\left(\partial^{\mu}+i \frac{\epsilon^{\mu \rho}}{\sqrt{g}} \partial_{\rho} \phi\right)\left(\partial^{\nu}+i \frac{\epsilon^{\nu \sigma}}{\sqrt{g}} \partial_{\sigma} \phi\right), \\
j^{\mu} & =i e_{b} \frac{1}{2}\left(\partial^{\mu}+i \frac{\epsilon^{\mu \rho}}{\sqrt{g}} \partial_{\rho} \phi\right),
\end{aligned}
$$

where $e_{b}$ is the charge of the boson. The normalization of stress tensor is consistent with that used in conformal field theory in two dimensions. We have kept the metric in these expressions for the stress tensor arbitrary, to enable us to evaluate the relevant Kubo formula. The partition function of the theory is identical to that of chiral Weyl fermions. The frequencies for the bosons in the Euclidean theory are even multiples of $\pi T$ and are given by

$$
\omega_{n}=2 n \pi T, \quad n \in Z .
$$

The thermal two point function for the bosons is given by

$$
S_{B}\left(\omega_{n}, p\right)=\left\langle\phi\left(\omega_{n}, p\right) \phi\left(-\omega_{n^{\prime}},-p^{\prime}\right)\right\rangle=\frac{2}{\left(i \omega_{n}\right)^{2}-p^{2}} 2 \pi \beta \delta_{n, n^{\prime}} \delta\left(p-p^{\prime}\right) .
$$

An important fact to be remembered while performing Wick contractions is that the one point function of the charge density in the thermal state is non zero in the presence of the chiral chemical potential and is given by

$$
\left\langle j^{t}(t, x)\right\rangle=e^{2} \frac{\mu_{-}}{2 \pi} .
$$

This can be derived from the partition function of the fermion theory which coincides with that of chiral bosons. ${ }^{9}$ One can show that the expectation value of the charge density is

\footnotetext{
${ }^{8} \epsilon^{\tau x}=\epsilon_{\tau x}=1$ and is anti symmetric in its indices.

${ }^{9}$ We review the thermodynamics of the Weyl gas section 5 .
} 
given by (2.75) also directly in the bosonic theory by adding the term $\mu_{-} j^{0}$ to the Hamiltonian and evaluating the partition function. Converting the expectation value of the charge to the Euclidean theory we obtain

$$
\left\langle j^{\tau}(\tau, x)\right\rangle=i e^{2} \frac{\mu_{-}}{2 \pi} .
$$

Let us convert this expectation value to a rule for determining one point functions of the operator $\phi$ in momentum space. Substituting the expression for $j^{\tau}$ from (2.72) and performing the Fourier transform to momentum space we obtain

$$
\left\langle\left(i \omega_{n}+p_{x}\right)\left\langle\phi\left(\omega_{n}, p\right)\right\rangle=2 \pi \beta \delta_{n, o} \delta(p) \frac{e^{2}}{e_{b}} \frac{\mu_{-}}{\pi} .\right.
$$

We will now determine the relation of the charge $e_{b}$ to the charge of the chiral fermion $e$ by demanding that the thermal expectation value of the stress-tensor $T^{\tau \tau}$ agrees with that of the Weyl fermions. The stress tensor in momentum space is given by

$$
\begin{aligned}
T_{f l}^{\tau \tau}\left(\omega_{n}, p\right)=\frac{1}{8 \beta} \sum_{n^{\prime}} \int \frac{d p^{\prime}}{2 \pi} & {\left.\left[i \omega_{n^{\prime}}-i \omega_{n}\right)+\left(p^{\prime}-p\right)\right]\left[i \omega_{n^{\prime}}+p^{\prime}\right] } \\
& \times \phi\left(\omega_{n^{\prime}}, p^{\prime}\right) \phi\left(\omega_{n}-\omega_{n^{\prime}}, p-p^{\prime}\right),
\end{aligned}
$$

The thermal expectation value can be written as,

$$
\begin{aligned}
&\left\langle T^{\tau \tau}\right\rangle=A+B . \\
&\left.A=\frac{1}{8 \beta} \sum_{n^{\prime}} \int \frac{d p^{\prime}}{2 \pi}\left[i \omega_{n^{\prime}}-i \omega_{n}\right)+\left(p^{\prime}-p\right)\right]\left[i \omega_{n^{\prime}}+p^{\prime}\right] \\
& \times\left\langle\phi\left(\omega_{n^{\prime}}, p^{\prime}\right) \phi\left(\omega_{n}-\omega_{n^{\prime}}, p-p^{\prime}\right)\right\rangle, \\
&\left.B=\frac{1}{8 \beta} \sum_{n^{\prime}} \int \frac{d p^{\prime}}{2 \pi}\left[i \omega_{n^{\prime}}-i \omega_{n}\right)+\left(p^{\prime}-p\right)\right]\left[i \omega_{n^{\prime}}+p^{\prime}\right] \\
& \times\left\langle\phi\left(\omega_{n^{\prime}}, p^{\prime}\right)\right\rangle\left\langle\phi\left(\omega_{n}-\omega_{n^{\prime}}, p-p^{\prime}\right)\right\rangle .
\end{aligned}
$$

We now evaluate term $A$ by substituting the propagator from (2.74), this yields

$$
\begin{aligned}
A & =\frac{1}{4 \beta} \sum_{n^{\prime}} \int \frac{d p^{\prime}}{2 \pi} \frac{\left[i \omega_{n^{\prime}}+p^{\prime}\right]^{2}}{\left(i \omega_{n^{\prime}}\right)^{2}-\left(p^{\prime}\right)^{2}} \\
& =-\frac{1}{4 \pi} \int_{-\infty}^{\infty} d p^{\prime} p^{\prime} b\left(p^{\prime}\right)
\end{aligned}
$$

Here we have factored out the $2 \pi \delta_{n, o} \delta(p)$ which results from momentum conservation. $b\left(p_{x}\right)$ denotes the Bose-Einstein distribution. To obtain the second equality we have used the following Matsubara sum for even integer frequencies

$$
\begin{aligned}
\sum_{\omega_{n^{\prime}}} \frac{1}{\beta} \frac{i \omega_{n^{\prime}}}{\left(i \omega_{n^{\prime}}-p_{x}^{\prime}\right)} & =-p_{x}^{\prime} b\left(p_{x}^{\prime}\right) \\
b\left(p_{x}^{\prime}\right) & =\frac{1}{e^{\beta p_{x}^{\prime}}-1} .
\end{aligned}
$$



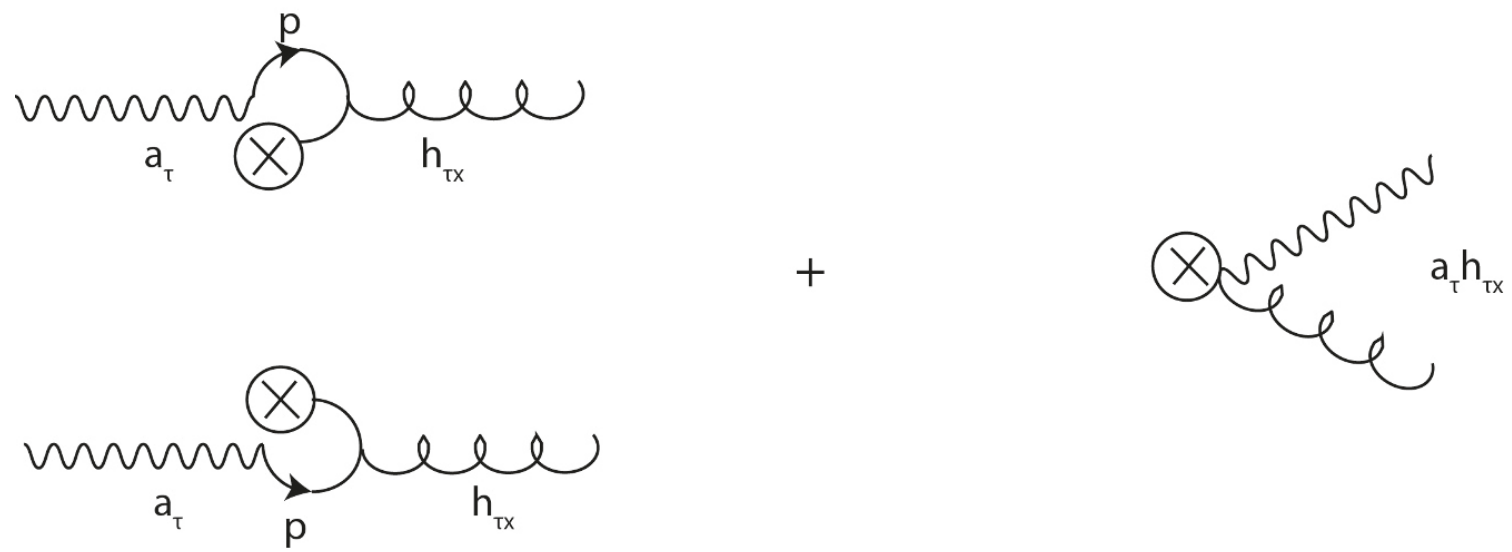

Figure 3. Diagrams contributing to $\zeta_{\text {Chiralboson }}^{(2)}$

On neglecting an infinite constant independent of temperature we obtain

$$
A=-\frac{1}{2 \pi} \int_{0}^{\infty} d p^{\prime} p^{\prime} b\left(p^{\prime}\right) .
$$

Using the first moment of the Bose-Einstein distribution given in (A.7) we obtain

$$
A=-\frac{\pi T^{2}}{12}
$$

Let us now examine the term $B$ in (2.80) Substituting (2.77) for the expectation values of the currents we obtain

$$
B=-\frac{1}{8}\left(\frac{e^{2}}{e_{g}} \frac{\mu_{-}}{\pi}\right)^{2}
$$

Here again we have factored out the delta function due to momentum conservation. Then putting terms $A$ and $B$ together we obtain

$$
\left\langle T^{\tau \tau}\right\rangle=-\frac{1}{4 \pi}\left(\frac{\pi^{2} T^{2}}{3}+\frac{e^{4} \mu_{-}^{2}}{e_{b}^{2} 2 \pi}\right) .
$$

Comparing this to the energy density of chiral fermions given in $(5.14)^{10}$ we see that for Bose-Fermi duality we must define the charge of the chiral boson to be given by

$$
e_{b}=\frac{e}{\sqrt{2 \pi}}
$$

Now that we have defined the theory we can proceed to evaluate the transport coefficients.

$\boldsymbol{\zeta}^{(2)}$ from chiral bosons. To obtain the possible contact terms, we expand the expression for for the $j^{\tau}$ component of the current, to the linear order in the metric. The relevant terms are given by

$$
j^{\tau}=\frac{i e_{b}}{2}\left(-\partial_{\tau}+i \partial_{x}\right) \phi-\frac{e_{b} h_{\tau x}}{4}\left(-\partial_{\tau}+i \partial_{x}\right) \phi+O\left(h, h^{2}\right) .
$$

\footnotetext{
${ }^{10}$ Note that the overall negative sign in $\left\langle T^{\tau \tau}\right\rangle$ is due to the fact we are in Euclidean space.
} 
Then the $\zeta^{(2)}$ is obtained by examining the following correlator

$$
\begin{aligned}
& \frac{1}{\sqrt{g}} \frac{\delta}{\delta h_{\tau x}(p)}\left(\frac{1}{\sqrt{g}} \frac{\delta \log Z}{\delta A_{\tau}(-p)}\right)=A+B, \\
& A=\left\langle j_{f l}^{\tau}(p) T_{f l}^{\tau x}(-p)\right\rangle, \quad B=-\left\langle\frac{\delta j^{\tau}(p)}{h_{\tau x}(p)}\right\rangle .
\end{aligned}
$$

Let us now evaluate the correlator $A$ by performing appropriate Wick's contractions. In momentum space we have

$$
\begin{aligned}
j_{f l}^{\tau}\left(\omega_{n}, p\right) & =\frac{i e_{b}}{2}\left(i \omega_{n}+p\right) \phi\left(\omega_{n}, p\right), \\
T_{f l}^{\tau x}\left(-\omega_{n},-p\right) & =\frac{i}{8 \beta} \sum_{n^{\prime}} \int \frac{d p^{\prime}}{2 \pi}\left(i \omega_{n^{\prime}}+p\right)\left[\left(i \tilde{\omega}_{n^{\prime}}+i \omega_{n}\right)+\left(p+p^{\prime}\right)\right] \\
& \times \phi\left(\tilde{\omega}_{n^{\prime}}, p^{\prime}\right) \phi\left(-\left(\omega_{n}+\omega_{n^{\prime}}\right),-\left(p+p^{\prime}\right)\right) .
\end{aligned}
$$

For the present correlator, due to the structure of the Wick contraction and the momentum and conservation, there is no sum over internal momenta. Evaluating the two possible Wick contractions we obtain

$$
A=\frac{e^{2} \mu_{-}}{4 \pi} \frac{i \omega_{n}+p}{p-i \omega_{n}}
$$

Here we have used the expectation value (2.77). The diagrams contributing to $A$ are shown on the l.h.s. of figure 3 . The contact term $B$ is given by

$$
B=\frac{e_{b}}{4}\left(i \omega_{n}+p\right)\left\langle\phi\left(\omega_{n}, p\right)\right\rangle=\frac{e^{2} \mu_{-}}{4 \pi},
$$

where again we have used (2.77). The diagram contributing to $B$ is shown on the r.h.s. of figure 3. Note that as before we have suppressed the $2 \pi \beta \delta(0)$ terms in $A$ and $B$. Adding the two contributions we obtain

$$
\left\langle j^{\tau}(p) T^{\tau x}(-p)\right\rangle=\frac{p}{p-i \omega_{n}} \frac{e^{2} \mu_{-}}{2 \pi} .
$$

This is identical to the expression obtained in the fermion language given in (2.48). Therefore taking the zero frequency limit first and then the zero momentum limit we see the contribution to the transport coefficient $\zeta^{(2)}$ from the chiral bosons is identical to that for the fermions and is given by

$$
\zeta_{\text {chiralboson }}^{(2)}=\frac{e^{2} \mu_{-}}{2 \pi} .
$$

Note that the contact terms contributed equally just an in the case of the chiral Weyl fermion.

$\lambda^{(2)}$ from chiral bosons. Let us expand the $T^{\tau \tau}$ component of the stress tensor to the linear order in the metric. The relevant terms to obtain the contribution of the contact terms are given by

$$
T^{\tau \tau}=\frac{-1}{8}\left(1+i h_{\tau x}\right)\left(-\partial_{\tau}+i \partial_{x}\right) \phi\left(-\partial_{\tau}+i \partial_{x}\right) \phi+O\left(h, h^{2}\right) .
$$



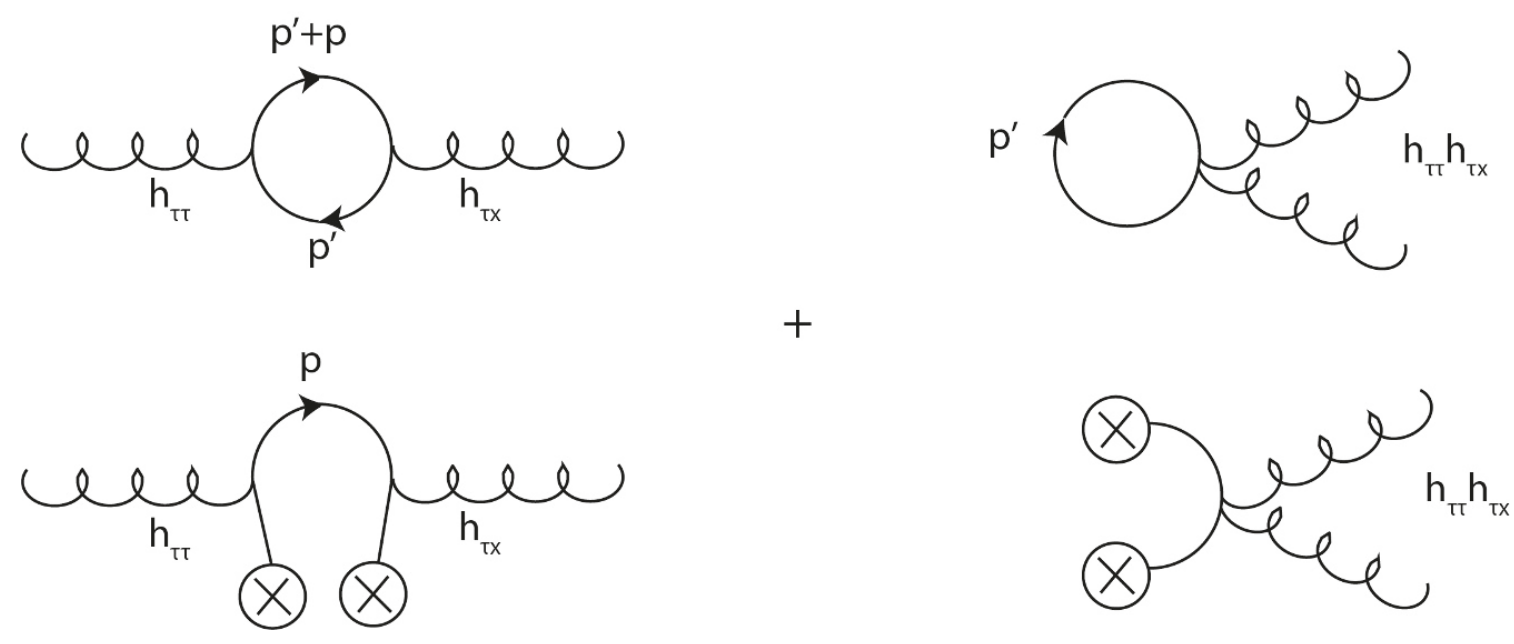

Figure 4. Diagrams contributing to $\lambda_{\text {Chiralboson }}^{(2)}$.

The correlator from which $\lambda^{(2)}$ is extracted is given by

$$
\begin{aligned}
& 2 \frac{1}{\sqrt{g}} \frac{\delta}{\delta h_{\tau x}(p)}\left(\frac{\delta \ln Z}{\delta h_{\tau \tau}(-p)}\right)=A+B \\
& A=\left\langle T_{f l}^{\tau \tau}(p) T_{f l}^{\tau x}(-p)\right\rangle, \quad B=-\left\langle\frac{\delta T^{\tau \tau}(p)}{\delta h_{\tau x}(p)}\right\rangle .
\end{aligned}
$$

In term $A$ and $B$ we need to evaluate all the contributing connected diagrams. The components of the flat space stress tensor in momentum space are given by

$$
\begin{gathered}
\left.T_{f l}^{\tau \tau}\left(\omega_{n}, p\right)=\frac{1}{8 \beta} \sum_{m} \int \frac{d p^{\prime}}{2 \pi}\left[i \omega_{m}-i \omega_{n}\right)+\left(p^{\prime}-p\right)\right]\left[i \omega_{m}+p^{\prime}\right] \\
\times \phi\left(\omega_{m}, p^{\prime}\right) \phi\left(\omega_{n}-\omega_{m}, p-p^{\prime}\right), \\
T_{f l}^{\tau x}\left(-\omega_{n},-p\right)=\frac{i}{8 \beta} \sum_{m} \int \frac{d p^{\prime}}{2 \pi}\left(i \omega_{m}+p^{\prime}\right)\left[\left(i \omega_{m}+i \omega_{n}\right)+\left(p+p^{\prime}\right)\right] \\
\times \phi\left(\omega_{m}, p^{\prime}\right) \phi\left(-\left(\omega_{n}+\omega_{m}\right),-\left(p+p^{\prime}\right)\right) .
\end{gathered}
$$

Let us proceed to evaluate the term $A$. This quantity has two contributions due to the fact that the current has an expectation value in the thermal state.

$$
\begin{gathered}
A=A_{1}+A_{2}, \\
A_{1}=\frac{2 i}{64 \beta} \sum_{n^{\prime}} \int \frac{d p^{\prime}}{2 \pi}\left(i \omega_{n^{\prime}}+p^{\prime}\right)^{2}\left(i \omega_{n}^{\prime}-\omega_{n}+p^{\prime}-p\right)^{2} S_{B}\left(\omega_{n^{\prime}}, p^{\prime}\right) S_{B}\left(\omega_{n}-\omega_{n^{\prime}}, p-p^{\prime}\right), \\
A_{2}=\frac{4 i}{64 \beta} \sum_{n^{\prime}} \int \frac{d p^{\prime}}{2 \pi}\left[\left(i \omega_{n^{\prime}}+p^{\prime}\right)^{2}\left(i \omega_{n}^{\prime}-\omega_{n}+p^{\prime}-p\right)^{2} S_{B}\left(\omega_{n^{\prime}}, p^{\prime}\right)\right. \\
\left.\times\left\langle\phi\left(\omega_{n}-\omega_{n}^{\prime}, p-p^{\prime}\right)\right\rangle\right]\left\langle\phi\left(\omega_{n^{\prime}}-\omega_{n}, p^{\prime}-p\right\rangle .\right.
\end{gathered}
$$

These two contributions are represented by the diagrams on the left hand side of figure 4 . Note that there are 2 possible Wick contractions which contribute equally giving rise to 
the factor 2 in $A_{1}$ and there are 4 possible Wick contractions which contribute equally giving rise to the factor 4 in $A_{2}$. Substituting the expression for the bosonic propagator $S_{B}$ from (2.74) in to $A_{1}$ we obtain

$$
A_{1}=\frac{i}{8 \beta} \sum_{n^{\prime}} \int \frac{d p^{\prime}}{2 \pi} \frac{\left(i \omega_{n^{\prime}}+p^{\prime}\right)\left(i \omega_{n^{\prime}}-i \omega_{n}+p^{\prime}-p\right)}{\left(i \omega_{m}-p^{\prime}\right)\left(\left(i \omega_{n^{\prime}}-i \omega_{n}\right)-\left(p^{\prime}-p\right)\right)} .
$$

We can now perform the Matsubara sum and take the zero frequency limit first and then zero momentum limit to obtain

$$
\begin{aligned}
\lim _{p \rightarrow 0, p_{0} \rightarrow 0} A_{1}\left(p_{0}, p\right) & =\frac{i}{2} \int \frac{d p^{\prime}}{2 \pi} p^{\prime} b\left(p^{\prime}\right), \\
& =\frac{i \pi T^{2}}{12} .
\end{aligned}
$$

From the structure of the Wick contractions in $A_{2}$ we see that it does not involve any Matsubara sum or integral. We need to use (2.77) to evaluate the expectation value of the currents and the relation between the charges in (2.86). This leads to the following value for $A_{2}$

$$
A_{2}=i \frac{e^{2} \mu_{-}}{4 \pi} .
$$

The contribution from the contact term $B$ is given by

$$
-\left\langle\frac{\delta T^{\tau \tau}(p)}{\delta h_{\tau x}(p)}\right\rangle=\frac{i}{4 \pi}\left(e^{2} \mu_{-}^{2}+\frac{\pi^{2} T^{2}}{3}\right) .
$$

This is easy to see from (2.94) since the contact term is equal to $-i\left\langle T^{\tau \tau}\right\rangle$. The diagrams contributing to $B$ are shown on the r.h.s. of figure 4. Considering the contributions both from the term $A$ and $B$ we obtain

$$
\lim _{p \rightarrow 0}\left\langle T_{f l}^{\tau \tau}(0, p) T_{f l}^{\tau x}(0-p)\right\rangle=\frac{i}{2 \pi}\left(e \mu_{-}^{2}+\frac{\pi^{2} T^{2}}{3}\right) .
$$

This is identical to that the contribution of the chiral fermions for the corresponding correlator as seen in (2.64). Therefore by the same logic we obtain the contribution of chiral bosons to $\lambda^{(2)}$ to be given by

$$
\lambda_{\text {chiral bosons }}^{(2)}=\frac{1}{4 \pi}\left(e^{2} \mu_{-}^{2}+\frac{\pi^{2} T^{2}}{3}\right) .
$$

It is indeed interesting to see how the different Feynman diagrams in the chiral boson theory which obeys the Bose-Einstein distribution organize themselves to give the same transport coefficients as that of the chiral fermion theory.

\subsection{Chiral gravitino like system}

It has been suspected that fermions with spin greater than $1 / 2$, for instance gravitinos, do not obey the replacement rule $[19,20]$. That is the relationship between parity odd transport and the microscopic anomaly breaks down. It will be instructive to see how 
this explicitly occurs in two dimensions, by performing a perturbative evaluation of the respective Kubo formula. There are no physical propagating gravitinos in two dimensions. However the following system mimics the features we require. Consider the Lagrangian

$$
\begin{aligned}
& S_{E}=S_{E}^{g}+S_{E}^{g h}, \\
& S_{E}=\int d \tau d x \sqrt{g} e_{a}^{\mu} \psi_{\rho}^{\dagger} \gamma^{a} D_{\mu} P_{-} \psi^{\rho},
\end{aligned}
$$

where

$$
D_{\mu} \psi^{\rho}=\partial_{\mu} \psi^{\rho}+\frac{1}{2} \omega_{\mu c d} \sigma^{c d} \psi^{\rho}+\Gamma_{\mu \sigma}^{\rho} \psi^{\sigma} .
$$

This Lagrangian in (2.104) is the gauged fixed Lagrangian of the gravitino in higher dimensions. The action $S_{E}^{g h}$ is the action of the corresponding ghosts. The contribution of the all the ghosts to the gravitational anomaly can be accounted by subtracting the contribution of a chiral spinor [23]. In two dimensions the above Lagrangian would not have any physical propagating degrees of freedom. We will see that in fact the action $S_{E}^{g}$ has central charge $c=-22$. However this gravitino like system will provide a simple illustrative example of how the replacement rule can break down. ${ }^{11}$

To proceed let us evaluate the central charge of this system. There are additional terms in the stress tensor due to the presence of the Christoffel symbol in the covariant derivative given in (2.105). The linearization of the Christoffel symbol results in

$$
\Gamma_{\rho \alpha}^{\mu}=\frac{1}{2} \eta^{\mu \sigma}\left(\partial_{\rho} h_{\sigma \alpha}+\partial_{\alpha} h_{\sigma \rho}-\partial_{\sigma} h_{\alpha \rho}\right)+O\left(h^{3}\right) .
$$

The stress tensor of the system is given by

$$
\begin{aligned}
T_{\mu \nu}^{g} & =T_{\mu \nu}^{g 1}+T_{\mu \nu}^{g 2}, \\
T_{\mu \nu}^{g 1} & =\frac{1}{2} \psi_{\rho}^{\dagger}(x)\left(\gamma_{\mu} \partial_{\nu}+\gamma_{\nu} \partial_{\mu}\right) P_{-} \psi^{\rho}(x) \\
T_{\mu \nu}^{g 2} & =-\frac{1}{2}\left(\partial _ { \sigma } \left(\psi^{\dagger, \sigma}(x) \gamma_{\mu} P_{-} \psi_{\nu}(x)-\partial_{\sigma}\left(\psi_{\mu}^{\dagger}(x) \gamma_{\nu} P_{-} \psi^{\sigma}(x)+(\mu \leftrightarrow \nu)\right) .\right.\right.
\end{aligned}
$$

Note that the total derivative term in the stress tensor arises from the contribution due to the linearization of the Christoffel symbol. While evaluating the stress tensor, we have used the equations of motion. To make it convenient to determine the central charge we go over to holomorphic coordinates defined by

$$
z=-i \tau+x, \quad \bar{z}=i \tau+x .
$$

Then the only non-trivial component of the stress tensor is given by

$$
T_{z z}(z)=\frac{1}{2}\left(\hat{\psi}_{\mu}^{*} \partial_{z} \hat{\psi}^{\mu}-\partial_{z} \hat{\psi}_{\mu}^{*} \psi^{\mu}\right)-i \epsilon^{\mu \nu} \partial_{z}\left(\hat{\psi}_{\mu}^{*} \hat{\psi}_{\nu}\right)
$$

Here $\hat{\psi}_{\mu}$ is the chiral component of the gravitino, $\epsilon$ is the anti-symmetric tensor defined with $\epsilon^{\tau x}=1$. We can now easily evaluate the central charge with the following OPE

$$
\hat{\psi}_{\mu}^{*}(z) \hat{\psi}^{\nu}(w)=\frac{\delta_{\mu}^{\nu}}{z-w} .
$$

\footnotetext{
${ }^{11}$ We thank R. Loganayagam and E. Witten for discussions which clarified our understanding of this point.
} 
On performing this, it is easy to see that there are additional contributions for the term proportional to the total derivative in (2.110). The central charge is given by

$$
c_{R}^{g 1}=2-24=-22 .
$$

The 2 results from the first term in (2.110), while the -24 results from the total derivative. Now to obtain the complete contribution, we need to take into the contribution from the ghosts, which is equivalent to subtracting the contribution from a complex chiral fermion. Therefore we obtain

$$
c_{R}=c_{R}^{g 1}+c_{R}^{g 2}=-22-1=-23 .
$$

The reason that this central charge is negative is due to the fact that the gravitino like system in 2 dimensions is not physical. The contribution of this gravitino like system to the gravitational anomaly is given by

$$
c_{g}^{\text {gravitino }}=-\frac{1}{96 \pi} c_{R}=\frac{23}{96 \pi} .
$$

Here we have used the relationship between the central charge and the coefficient of gravitational anomaly form [7]. This contribution to the gravitational anomaly of the gravitino like system coincides with what has been considered as the contribution of the 'gravitino' in [19].

$\lambda^{(2)}$ from gravitino like system. We now evaluate the contribution to the transport coefficient $\lambda^{(2)}$ from the gravitino like system. The components of the classical stress tensor for this system in momentum space can be read out from (2.107), they are given by

$$
\begin{aligned}
T_{f l}^{\tau \tau}(p)= & T_{f l(1)}^{\tau \tau}+T_{f l(2)}^{\tau \tau}, \\
T_{f l(1)}^{\tau \tau}(p)= & \int \frac{d^{2} p^{\prime}}{(2 \pi)^{2}}\left(\psi_{\mu}^{\dagger}\left(p^{\prime}-p\right) \gamma^{\tau}\left(i p_{\tau}\right) P_{-} \psi^{\mu}\left(p^{\prime}\right)\right) \\
T_{f l(2)}^{\tau \tau}(p)= & i p_{\sigma} \int \frac{d^{2} p^{\prime}}{(2 \pi)^{2}}\left[\left(\psi^{\dagger \sigma}\left(p^{\prime}-p\right) \gamma^{\tau} P_{-} \psi^{\tau}\left(p^{\prime}\right)\right)-\left(\psi^{\dagger \tau}\left(p^{\prime}-p\right) \gamma^{\tau} P_{-} \psi^{\sigma}\left(p^{\prime}\right)\right)\right] \\
T_{f l}^{\tau x}(-p)= & T_{f l(1)}^{\tau x}+T_{f l(2)}^{\tau x}, \\
T_{f l(1)}^{\tau x}(-p)= & \left.\int \frac{d^{2} p^{\prime}}{(2 \pi)^{2}} \frac{1}{2}\left(\psi_{\mu}^{\dagger}\left(p^{\prime}+p\right)\left(\gamma^{\tau} i p_{x}^{\prime}+\gamma^{x} i p_{\tau}\right) P_{-} \psi^{\mu}\left(p^{\prime}\right)\right)\right) \\
T_{f l(2)}^{\tau x}(-p)= & -\frac{1}{2}\left(i p_{\sigma}\right) \int \frac{d^{2} p^{\prime}}{(2 \pi)^{2}}\left[\left(\psi^{\dagger \sigma}\left(p^{\prime}+p\right) \gamma^{\tau} P_{-} \psi^{x}\left(p^{\prime}\right)+\psi^{\dagger \sigma}\left(p^{\prime}+p\right) \gamma^{x} P_{-} \psi^{\tau}\left(p^{\prime}\right)\right)\right. \\
& \left.-\left(\psi^{\dagger \tau}\left(p^{\prime}+p\right) \gamma^{x} P_{-} \psi^{\sigma}\left(p^{\prime}\right)+\psi^{\dagger x}\left(p^{\prime}+p\right) \gamma^{\tau} P_{-} \psi^{\sigma}\left(p^{\prime}\right)\right)\right]
\end{aligned}
$$

Here the terms in the stress tensor, which arises from the total derivative, have been separated and their contribution is labeled by the subscript (2). The two point function of the classical part stress tensor is given by

$$
\left\langle T_{f l}^{\tau \tau}(p) T_{f l}^{\tau x}(-p)\right\rangle=\left\langle T_{f l(1)}^{\tau \tau}(p) T_{f l(1)}^{\tau x}(-p)\right\rangle+\left\langle T_{f l(2)}^{\tau \tau}(p) T_{f l(2)}^{\tau x}(-p)\right\rangle .
$$

It can be easily seen from the structure of the Wick contractions, all the remaining cross terms vanish. Now the contribution from $\left\langle T_{f l(1)}^{\tau \tau}(p) T_{f l(1)}^{\tau x}(-p)\right\rangle$ is twice that of a single 
chiral fermion. We will show that the contribution from $\left\langle T_{f l(2)}^{\tau \tau}(p) T_{f l(2)}^{\tau x}(-p)\right\rangle$ vanishes. We evaluate this correlator by performing Wick contractions using the propagator

$$
\left\langle\psi_{\mu}\left(\omega_{m}, p\right) \psi_{\nu}^{\dagger}\left(\omega_{m^{\prime}}, p^{\prime}\right)=2 \pi \beta \delta\left(p-p^{\prime}\right) \eta_{\mu \nu} \delta_{m, m^{\prime}}\left(\begin{array}{cc}
0 & \frac{-i}{i \omega_{m}-p} \\
\frac{-i}{i \omega_{m}+p} & 0
\end{array}\right) .\right.
$$

Note that we have considered the gravitino like theory at finite temperature but not at finite chemical potential. This is sufficient to pick out the pure gravitational contribution to the transport coefficient $\lambda^{(2)}$. After performing the Matsubara sums involved we obtain

$$
\begin{aligned}
\left\langle T_{f l(2)}^{\tau \tau}\left(\omega_{m}, p\right) T_{f l(2)}^{\tau x}(-\omega,-p)\right\rangle & =i p \frac{i \omega_{m}+p}{i \omega_{m}-p} \int_{-\infty}^{\infty} \frac{d p^{\prime}}{2 \pi}\left(f(p)-f\left(p^{\prime}+p\right)\right), \\
& =-i \frac{p^{2}}{2 \pi} \frac{i \omega_{m}+p}{i \omega_{m}-p}
\end{aligned}
$$

Therefore we see that in the zero momentum limit the contribution from the Christoffel symbol vanishes in the zero momentum limit.

$$
\lim _{p \rightarrow 0, \omega_{m} \rightarrow 0}\left\langle T_{f l(2)}^{\tau \tau}\left(\omega_{m}, p\right) T_{f l(2)}^{\tau x}(-\omega,-p)\right\rangle=0 .
$$

For later analysis it is important to note that even if we did not evaluate the integral in (2.119), as long as one is interested in the finite piece of the integral, taking the zero frequency limit and then the zero momentum limit ensures that the this contribution vanishes. Thus we conclude that the contribution to the transport coefficient $\lambda^{(2)}$ of the total derivative term in the classical stress tensor (2.107), which arises due to the presence of the Christoffel symbol, vanishes. Let us now examine possible contact terms which arise from expanding the Christoffel symbol. On performing the expansion of the vierbein, metric as done in (2.25), (2.26) and (2.27) as well as the term involving the Christoffel symbol in the action (2.104), the relevant contact terms arise from

$$
S_{\text {Christoffell }}^{(1)}=\int d \tau d x\left(h_{\tau x} \partial_{x} h_{\tau \tau}\left(\psi_{x}^{\dagger} \gamma^{\tau} \psi_{x}\right)+\frac{h_{\tau x} \partial_{\tau} h_{\tau \tau}}{2}\left(\psi_{\tau}^{\dagger} \gamma^{x} \psi_{\tau}\right)-\frac{h_{\tau x} \partial_{x} h_{\tau \tau}}{2}\left(\psi_{\tau}^{\dagger} \gamma^{\tau} \psi_{\tau}\right)\right)
$$

Note that the origin of all these terms is from the expansion of the Christoffel symbol and therefore they involve a derivative on the metric. This is unlike the other terms in the second line of $S^{(1)}$ given in (2.28), which involve a derivative on the fermion. The presence of the derivative on the metric leads to a momentum factor outside the Fermi-Dirac integral after performing the Wick contraction and the Matsubara sum. Therefore again, on taking the zero frequency and zero momentum limit, the contributions of these terms vanish.

The above analysis shows that the terms arising from the Christoffel symbol in the action for the gravitino like system in (2.104), do not contribute to the transport coefficient $\lambda^{(2)}$. Thus the contribution of this system to this transport coefficient is identical to two chiral Weyl fermions. We then have to subtract the ghost contribution which is equal to a single chiral Weyl fermion. The net result is that $\lambda^{(2)}$ for a gravitino like system is given by

$$
\lambda^{(2)}=\frac{\pi T^{2}}{12} .
$$


Therefore we have

$$
\tilde{c}_{2 d}^{\text {gravitino }}=\frac{\pi}{12}
$$

Comparing the value of $c_{g}^{\text {gravitino }}$ for the system in (2.114), we see that this system does not satisfy the replacement rule obtained by consistency of the Euclidean vacuum since $\tilde{c}_{2 d}^{\text {gravitino }} \neq-8 \pi^{2} c_{g}^{\text {gravitino }}$.

At this point it is important to make the following observations. The terms proportional to the Christoffel symbol contribute to the anomaly coefficient. However for the transport coefficient the contributions of this vanished on taking the zero momentum limit. It is for this reason that for the purpose of evaluating the transport coefficient the system along with the ghosts behaved like a single chiral Weyl fermion. Though the system we analyzed in two dimensions possibly does not arise naturally in any physical system since the central charge of this system is negative, it illustrates the mechanism of how for higher spin chiral fermions the transport coefficient is not constrained by the microscopic anomaly. We will return to this phenomenon again in $6 \mathrm{~d}$ dimensions, where chiral gravitinos are physical and they contribute to the pure gravitational anomaly.

\section{Anomalies and transport in $d=4$}

Let us begin by parametrizing the constitutive for the stress tensor and the charge current. We will restrict our attention to terms occurring in first order in the derivative expansion in the anomaly frame. In $d=4$ the constitutive relations are given by

$$
\begin{aligned}
T^{\mu \nu}= & (\epsilon+P) u^{\mu} u^{\nu}-P \eta^{\mu \nu}+\lambda_{1}^{(4)}\left(u^{\mu} \frac{1}{2} \epsilon^{\nu \rho \alpha \beta} u_{\rho} F_{\alpha \beta}+u^{\nu} \frac{1}{2} \epsilon^{\mu \rho \alpha \beta} u_{\rho} F_{\alpha \beta}\right), \\
& +\lambda_{2}^{(4)}\left(u^{\mu} \epsilon^{\nu \rho \alpha \beta} u_{\rho} \partial_{\alpha} u_{\beta}+u^{\nu} \epsilon^{\mu \rho \alpha \beta} u_{\rho} \partial_{\alpha} u_{\beta}\right), \\
j^{\mu}= & n u^{\mu}+\zeta_{1}^{(4)} \frac{1}{2} \epsilon^{\mu \rho \alpha \beta} u_{\rho} F_{\alpha \beta}+\zeta_{2}^{(4)} \epsilon^{\mu \rho \alpha \beta} u_{\rho} \partial_{\alpha} u_{\beta} .
\end{aligned}
$$

where, $\epsilon, p, n, \mu, \lambda_{1}, \lambda_{2}, \zeta_{1}, \zeta_{2}$ refer to the energy density, pressure, charge density, chemical potential and the four anomalous transport coefficients respectively. The metric, we work in, has the mostly negative signature $g_{\mu \nu}=(1,-1,-1,-1)$. Let us derive the Kubo formulae for the parity odd transport coefficients, by considering perturbations of the fluid from the rest frame. Let the velocity of fluid be given by $u^{\mu}=\left(1,0, v^{y}, 0\right)$ with $\left|v^{y}\right| \ll 1$, we also have such that $u^{\mu} u_{\mu}=1+O\left(v^{2}\right)$. The gauge field, velocity and metric perturbations are given by,

$$
\begin{aligned}
A_{\mu} & =\left(0,0, a_{y}, 0,\right) & u_{\mu} & =\left(0,0,-v^{y}+h_{t y}, 0\right), \\
g_{\mu \nu} & =\eta_{\mu \nu}+h_{\mu \nu}, & h_{t y} & =h_{t y} \neq 0 .
\end{aligned}
$$

All perturbations are assumed to depend on only the $z$ direction and we can work with the Fourier modes in this direction. Expanding the constitutive relation to the linear order and interpreting it as an Ward identity, just as it was done in the case of $d=2$, we obtain the following Kubo formula for the anomalous transport coefficients.

$$
\lambda_{1}^{(4)}=\lim _{p_{z} \rightarrow 0, p_{0} \rightarrow 0} \frac{1}{i p_{z}}\left\langle T^{t x}\left(p_{0}, p_{z}\right) j^{y}\left(-p_{0},-p_{z}\right)\right\rangle_{R},
$$




$$
\begin{aligned}
\lambda_{2}^{(4)} & =\lim _{p_{z} \rightarrow 0, p_{0} \rightarrow 0} \frac{1}{i p_{z}}\left\langle T^{t x}\left(p_{0}, p_{z}\right) T^{t y}\left(-p_{0},-p_{z}\right)\right\rangle_{R} \\
\zeta_{1}^{(4)} & =\lim _{p_{z} \rightarrow 0, p_{0} \rightarrow 0} \frac{1}{i p_{z}}\left\langle j^{x}\left(p_{0}, p_{z}\right) j^{y}\left(-p_{0},-p_{z}\right)\right\rangle_{R}, \\
\zeta_{2}^{(4)} & =\lim _{p_{z} \rightarrow 0, p_{0} \rightarrow 0} \frac{1}{i p_{z}}\left\langle j^{x}\left(p_{0}, p_{z}\right) T^{t y}\left(-p_{0},-p_{z}\right)\right\rangle_{R} .
\end{aligned}
$$

The expectation values in all these correlators refer to real time retarded correlators at finite temperature and chemical potential. As discussed earlier, we have to take the zero frequency limit first and then the zero momentum limit. All of these correlators involve division by a power of momentum, since these transport coefficients occur at the first order in the derivative expansion. Finally note that the transport coefficients $\lambda_{1}^{(4)}$ and $\zeta_{2}^{(4)}$ are identical and therefore it is sufficient to evaluate only 3 of the Kubo formulae in (3.3). As done for the case of $d=2$ to obtain the Minkowski real time retarded correlators, we will evaluate the corresponding correlator in the Euclidean theory and then analytically continue to obtain the transport coefficients.

Before proceeding, let us recall previous evaluations of these transport coefficients in the literature. For a theory of Weyl fermions, the coefficient $\zeta_{1}^{(4)}$ was first evaluated in [21], the coefficient $\zeta_{1}^{(4)}$ as well as $\zeta_{2}^{(4)}$ was evaluated in [10]. As we will see, the coefficient $\lambda_{1}^{(4)}$ is sensitive to the mixed gravitational anomaly in the theory and this was obtained in [8]. The evaluation of the coefficient $\lambda_{2}^{(4)}$ was begun in [10], and all the diagrams including the contact terms which contribute to this coefficient was finally done in [22]. The fact that the mixed anomaly contributes to $\lambda_{1}^{(4)}$, was mentioned in [10]. However the complete evaluation of the contribution of the mixed anomaly coefficient including the contact terms has not been done. The work in [22] was interested only in the contribution of the global gauge anomaly. We will complete this small gap in literature, we will also develop a simple method of evaluating the resulting angular integrals. Our calculation will also serve as a cross check of the methods in [22] which are different from that in this paper. We also show that we can take the zero frequency and zero momentum limit first and then perform the resulting angular integrals. This considerably simplifies the evaluation of these integrals. In all the previous work, the angular integrals were first performed, in fact [22] provides a formula which was guessed for angular integrals which occur in arbitrary dimensions.

\subsection{Chiral fermions}

Let us proceed to evaluate the corresponding correlators first, in the Euclidean theory of free chiral Weyl fermions in $d=4$. Following the set up in section (2), the expression for the Euclidean $\left\langle j^{\mu} j^{\nu}\right\rangle_{E}$ is given by

$$
\begin{aligned}
\left\langle j^{\mu} j^{\nu}\right\rangle_{E, c l}= & -\left\langle j^{\mu}\right\rangle_{E}\left\langle j^{\nu}\right\rangle_{E}+\frac{1}{\mathcal{Z}_{E}^{(0)}} \int \mathcal{D} \psi^{\dagger} \mathcal{D} \psi e^{S_{E}^{(0)}} \frac{\delta S_{E}}{\delta a_{\mu}} \frac{\delta S_{E}}{\delta a_{\nu}} \\
& +\frac{1}{\mathcal{Z}_{E}^{(0)}} \int \mathcal{D} \psi^{\dagger} \mathcal{D} \psi e^{S_{E}^{(0)}} \frac{\delta^{2} S_{E}}{\delta a_{\mu} \delta a_{\nu}},
\end{aligned}
$$

while the two point functions $\left\langle j^{\mu} j^{\nu}\right\rangle_{E}$ and $\left\langle j^{\mu} T^{\nu \rho}\right\rangle^{e}$ are given in (2.15) and (2.16) respectively. For convenience, let us define the following coefficients, which are obtained from 
taking limits of the Euclidean correlators

$$
\begin{aligned}
\tilde{\zeta}_{1}^{(4)} & =\lim _{p_{z} \rightarrow 0} \frac{1}{i p_{z}}\left\langle j^{x}\left(0, p_{z}\right) j^{y}\left(0,-p_{z}\right)\right\rangle_{E}, \\
\tilde{\zeta}_{2}^{(4)} & =\lim _{p_{z} \rightarrow 0} \frac{1}{i p_{z}}\left\langle j^{x}\left(0, p_{z}\right) T^{\tau y}\left(0,-p_{z}\right)\right\rangle_{E}, \\
\tilde{\lambda}_{2}^{(4)} & =\lim _{p_{z} \rightarrow 0} \frac{1}{i p_{z}}\left\langle T^{\tau x}\left(0, p_{z}\right) T^{\tau y}\left(0,-p_{z}\right)\right\rangle_{E} .
\end{aligned}
$$

To obtain the transport coefficients from the above definitions, we use the relation between Minkowski real time retarded correlators and the Euclidean correlators which are given by

$$
\begin{aligned}
\left\langle j^{x}\left(\omega_{n}, p\right) j^{y}\left(-\omega_{n},-p\right)\right\rangle_{E} & =\left.\left\langle j^{x}\left(p_{0}, p\right) j^{y}\left(-p_{0},-p\right)\right\rangle_{R}\right|_{i \omega \rightarrow p_{0}+i \epsilon}, \\
\left\langle j^{x}\left(\omega_{n}, p\right) T^{\tau y}\left(-\omega_{n},-p\right)\right\rangle_{E} & =\left.i\left\langle j^{x}\left(p_{0}, p\right) T^{t y}\left(-p_{0},-p\right)\right\rangle_{R}\right|_{i \omega \rightarrow p_{0}+i \epsilon}, \\
\left\langle T^{\tau x}\left(\omega_{n}, p\right) T^{\tau y}\left(-\omega_{n},-p\right)\right\rangle_{E} & =-\left.\left\langle T^{t x}\left(p_{0}, p\right) T^{t y}\left(-p_{0},-p\right)\right\rangle_{R}\right|_{i \omega \rightarrow p_{0}+i \epsilon} .
\end{aligned}
$$

where, $\omega_{n}=2 \pi n T$, are the integer quantized Matsubara frequencies. Using (3.6) and the definitions of the transport coefficients in (3.3) and (3.5) we obtain

$$
\tilde{\zeta}_{1}^{(4)}=\zeta_{1}^{(4)}, \quad \tilde{\zeta}_{2}^{(4)}=i \zeta_{2}^{(4)}, \quad \tilde{\lambda}_{2}^{(4)}=-\lambda_{2}^{(4)} .
$$

For Weyl fermions in $d=4$, the partition function $\mathcal{Z}_{E}$ is given by

$$
\mathcal{Z}_{E}=\int \mathcal{D} \psi_{E}^{\dagger} \mathcal{D} \psi_{E} \exp \left(S_{E}\right)
$$

where,

$$
S_{E}=\int d \tau d^{3} x \sqrt{g} e_{a}^{\mu} \psi^{\dagger} \gamma^{a} D_{\mu} P_{-} \psi
$$

The covariant derivative is defined as

$$
D_{\rho} \psi=\partial_{\rho} \psi+\frac{1}{2} \omega_{\rho a b} \sigma^{a b} \psi+i e A_{\rho} \psi
$$

where, $\omega_{\rho a b}$ is the spin connection and $\sigma^{a b}=\frac{1}{4}\left[\gamma_{a}, \gamma_{b}\right]$. Unlike $d=2$, the contributions from the term proportional to the spin connection does not vanish for $d>2$. We work in Euclidean space where the gamma matrices satisfy,

$$
\left\{\gamma^{a}, \gamma^{b}\right\}=2 \eta_{E}^{a b}, \quad \eta_{E}^{a b}=-\delta^{a b}
$$

and

$$
P_{ \pm}=\frac{1}{2}\left(1 \pm \gamma_{5}\right)
$$

Note raising and lowering of flat space indices are done by $\eta_{E}^{a b}$. We now perturb the action in (3.9) from flat space and constant chemical potential, by considering the expansion with the following choice of metric and gauge field perturbations,

$$
g_{\mu \nu}=-\delta_{\mu \nu}+h_{\mu \nu}, \quad A_{\mu}=A_{\mu}^{(0)}+a_{\mu},
$$


where $A_{\tau}^{(0}$ refers to the background chemical potential. For this metric perturbation following [23], we can work with the following vierbein to the linear order in fluctuations

$$
e_{a \mu}=-\delta_{a \mu}+\frac{h_{a \mu}}{2}+O\left(h^{2}\right) .
$$

Essentially we are working in a gauge which ensures that the vierbein is symmetric. Expanding the action to the quadratic order in the fluctuations we obtain

$$
\begin{aligned}
S_{E} & =S_{E}^{(0)}+S_{E}^{(1)}+S_{E}^{(2)}, \\
S_{E}^{(0)} & =\int d^{4} x \psi_{E}^{\dagger}\left(\gamma^{\tau} D_{\tau}+\gamma^{i} \partial_{i}\right) P_{-} \psi_{E}, \\
S_{E}^{(1)} & =-\int d^{4} x \frac{h_{\mu \nu}}{4} \psi_{E}^{\dagger}\left(\gamma^{\mu} \partial^{\nu}+\gamma^{\nu} \partial^{\mu}\right) P_{-} \psi_{E}+i e \int d^{4} x \psi_{E}^{\dagger} \gamma^{\mu} A_{\mu} P_{-} \psi_{E}, \\
S_{E}^{(2)} & =\int d^{4} x \frac{h_{\lambda \alpha} \partial_{\mu} h_{\nu \alpha}}{16} \psi_{E}^{\dagger} \Gamma^{\mu \lambda \nu} P_{-} \psi_{E}+O\left(h^{3}\right) .
\end{aligned}
$$

Here coefficient of the term linear in the metric is the stress tensor in flat space and the coefficient of the term linear in the gauge field is the charge current. The second order terms in the the metric, given in the last term of (3.15), arises from expanding of the spin connection, the repeated indices in the subscript refer to summation. This term contributes to the mixed anomaly coefficient [23]. We will see that that this term gives rise to a contact term and it is important in the evaluation of the coefficient $\lambda_{2}^{(4)}$. $\Gamma^{\mu \nu \alpha}$ stands for totally anti-symmetric combination of gamma matrices which is given by

$$
\Gamma^{\mu \nu \alpha}=\frac{1}{3 !}\left(\gamma^{\mu} \gamma^{\nu} \gamma^{\alpha}-\gamma^{\nu} \gamma^{\mu} \gamma^{\alpha}+(\text { cyclic })\right) .
$$

Using the action (3.15), we can obtain the stress tensor and charge current which are given by

$$
\begin{aligned}
T_{f l}^{\mu \nu}(x) & =\frac{1}{2} \psi_{E}^{\dagger}\left(\gamma^{\mu} \partial^{\nu}+\gamma^{\nu} \partial^{\mu}\right) P_{-} \psi_{E}, \\
j_{f l}^{\mu} & =-i e \psi_{E}^{\dagger} \gamma^{\mu} P_{-} \psi_{E} .
\end{aligned}
$$

We now take the background chemical potential to be

$$
A_{\tau}^{(0)}=i \mu+i \mu_{c} \gamma_{5} .
$$

The propagator derived from the action (3.15) is given by

$$
\begin{aligned}
S_{E}(q) & =\frac{-i}{\gamma^{\tau}\left(w_{m}-i e \mu-i e \mu_{c} \gamma_{5}\right)+\gamma^{i} q_{i}}, \\
& =\frac{-i}{2} \sum_{s, t= \pm} \Delta_{t}\left(i \omega_{s}, q\right) P_{s} \gamma_{\mu} \hat{q}_{t}^{\mu}
\end{aligned}
$$

where

$$
\begin{array}{rlrl}
P_{ \pm} & =\frac{\left(1 \pm \gamma_{5}\right)}{2}, & \hat{q}_{ \pm}^{\mu}=(-i, \pm \hat{q}), & E_{q}=|q| \\
\Delta_{ \pm}\left(q_{0}, q\right)=\frac{1}{q_{0} \mp E_{q}}, & \omega_{s}=\omega_{m}-i e \mu-\operatorname{sie} \mu_{c}, & \mu_{ \pm}=\mu \pm \mu_{c} .
\end{array}
$$




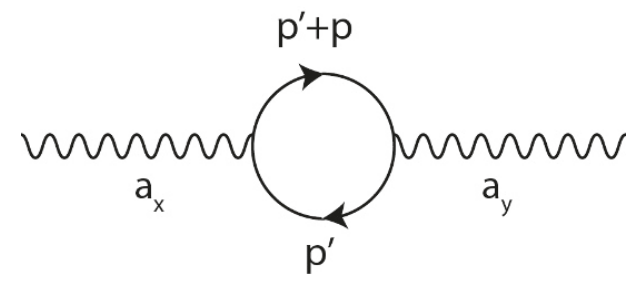

Figure 5. Diagram contributing to $\zeta_{1}^{(4)}$.

Evaluation of $\zeta_{\mathbf{1}}^{(4)}$. From the Kubo formula in (3.5), the transport coefficient $\tilde{\zeta}_{1}^{(4)}$ is given by

$$
\zeta_{1}^{(4), E}=\lim _{p_{z} \rightarrow 0} \frac{1}{i p_{z}}\left\langle j^{x}\left(0, p_{z}\right) j^{y}\left(0,-p_{z}\right)\right\rangle .
$$

For all calculations of correlators in the rest of the paper, we will set the frequency to zero at the beginning. The correlator is defined using (3.4) and from the expansion of the action given in (3.15), we see that there are no contact terms that contribute to this correlator. Converting the charge current given in $((3.19))$ to momentum space we obtain

$$
j_{f l}^{\mu}(0, p)=-i e \frac{1}{\beta} \sum_{m} \int \frac{d^{3} p^{\prime}}{(2 \pi)^{3}} \psi_{E}^{\dagger}\left(\omega_{m}, p^{\prime}-p\right) \gamma^{\mu} P_{-} \psi_{E}\left(\omega_{m}, p^{\prime}\right)
$$

After performing Wick contractions the correlator of interest is given by

$$
\begin{aligned}
I(p) & =\frac{1}{i p_{z}}\left\langle j^{x}(0, p) j^{y}(0,-p)\right\rangle, \\
& =\frac{e^{2}}{i p_{z} \beta} \sum_{m} \int \frac{d^{3} p_{1}}{(2 \pi)^{3}} \operatorname{Tr}\left[\gamma^{x} S_{E}\left(\omega_{m}, p^{\prime}+p\right) \gamma^{y} S_{E}\left(\omega_{m}, p\right) P_{+}\right] .
\end{aligned}
$$

Using standard methods to sum over the Matsubara frequencies we obtain

$$
\begin{gathered}
\sum_{m} \frac{1}{\beta} \frac{1}{\left.\left(i \omega_{m}+e \mu_{-}-t E_{p}\right)\right)\left(i \omega_{m}+e \mu_{-}+i \omega_{n}-u E_{p+q}\right)} \\
=\frac{t f\left(E_{p}-t e \mu_{-}\right)-u f\left(E_{p+q}-u e \mu_{-}-i \omega_{n}\right)}{i \omega_{n}+t E_{p}-u E_{p+q}} \\
\sum_{m} \frac{1}{\beta} \frac{i \omega_{m}+e \mu_{-}}{\left.\left(i \omega_{m}+e \mu_{-}-t E_{p}\right)\right)\left(i \omega_{m}+e \mu_{-}+i \omega_{n}-u E_{p+q}\right)} \\
=\frac{E_{p} f\left(E_{p}-t e \mu_{-}\right)-E_{p+q} f\left(E_{p+q}-u e \mu_{-}-i \omega_{n}\right)}{i \omega_{n}+t E_{p}-u E_{p+q}} \\
\sum_{m} \frac{1}{\beta} \frac{\left(i \omega_{m}+e \mu_{-}\right)^{2}}{\left.\left(i \omega_{m}+e \mu_{-}-t E_{p}\right)\right)\left(i \omega_{m}+e \mu_{-}+i \omega_{n}-u E_{p+q}\right)} \\
=\frac{t E_{p}^{2} f\left(E_{p}-t e \mu_{-}\right)-u E_{p+q}^{2} f\left(E_{p+q}-u e \mu_{-}-i \omega_{n}\right)}{i \omega_{n}+t E_{p}-u E_{p+q}} .
\end{gathered}
$$

While performing all these sums, we have ignored terms which are independent of temperature and chemical potential. Using the results for the sums as well as performing the 
trace in $(3.25)$, we obtain

$$
I\left(p_{z}\right)=\sum_{t, u= \pm} \frac{e^{2}}{2} \int \frac{d^{3} p^{\prime}}{(2 \pi)^{3}} \frac{t}{E_{p^{\prime}+p}} \frac{t f\left(E_{p^{\prime}+p}-t e \mu_{-}\right)-u f\left(E_{p^{\prime}}-u e \mu_{-}\right)}{t E_{p^{\prime}+p}-u E_{p^{\prime}}} .
$$

After changing the variable of integration in the first term of the above equation, we obtain

$$
I\left(p_{z}\right)=\sum_{t, u= \pm} \frac{e^{2}}{2} \int \frac{d^{3} p^{\prime}}{(2 \pi)^{3}}\left(\frac{f\left(E_{p^{\prime}}-t e \mu_{-}\right)}{E_{p^{\prime}}\left(t E_{p^{\prime}}-u E_{p^{\prime}+p}\right)}+\frac{t u f\left(E_{p^{\prime}}-u e \mu_{-}\right)}{E_{p^{\prime}+p}\left(u E_{p^{\prime}}-t E_{p^{\prime}+p}\right)}\right) .
$$

Summing over $u$ in the first term and over $t$ in the second term, we get,

$$
\begin{aligned}
I\left(p_{z}\right) & =2 e^{2} \int \frac{d^{3} p_{1}}{(2 \pi)^{3}} \frac{t f\left(E_{p^{\prime}}-t e \mu_{-}\right)}{\left(E_{p^{\prime}}^{2}-E_{p^{\prime}+p}^{2}\right)} \\
& =-\frac{2 e^{2}}{(2 \pi)^{2}} \sum_{t= \pm} \int d p^{\prime} p^{\prime 2} \sin \theta t f\left(E_{p^{\prime}}-t e \mu_{-}\right) \frac{1}{\left(p^{2}+2 p^{\prime} p_{z} \cos \theta\right)} .
\end{aligned}
$$

At this stage we need to perform the angular integral. This integral can of course be easily performed, however we will show that we can take the $p_{z} \rightarrow 0$ limit first and then perform the integral. Though the advantage of this procedure is limited in $d=4$, we will see that for $d=6$ this procedure simplifies the resulting integrals considerably. Expanding in $p_{z}$ we obtain

$$
I\left(p_{z}\right)=-\frac{2 e^{2}}{(2 \pi)^{2}} \sum_{t= \pm} \int d p^{\prime} p^{2} \sin \theta t f\left(E_{p^{\prime}}-t e \mu_{-}\right)\left(\frac{1}{2 p^{\prime} p_{z} \cos \theta}-\frac{1}{4 p^{\prime 2} \cos ^{2} \theta}+O\left(p_{z}^{2}\right)\right) .
$$

After a change of variables $z=\cos \theta$, the required integrals are of the form

$$
J_{1}=\int_{0}^{\pi} \frac{\sin \theta d \theta}{\cos \theta}=\int_{-1}^{1} \frac{d z}{z}, \quad J_{2}=\int_{0}^{\pi} \frac{\sin \theta d \theta}{\cos ^{2} \theta}=\int_{-1}^{1} \frac{d z}{z^{2}} .
$$

The integrals are all on the real line, we make these integrals well defined by shifting the integrand to $z \rightarrow z+i \epsilon$. Then we obtain

$$
J_{1}=0, \quad J_{2}=-2 .
$$

Such a prescription for doing the resulting integrals renders each term in the expansion in $p_{z}$ finite. In fact it is easy to check that performing the angular integral first and then expanding in $p_{z}$, gives identical result to expanding in $p_{z}$ first and then doing the integrals term by term using the $i \epsilon$ prescription. From these results for the integral, we obtain

$$
\begin{aligned}
\tilde{\zeta}_{1}^{(4)}=\lim _{p_{z} \rightarrow 0} \frac{1}{i p_{z}}\left\langle j^{x}(p) j^{y}(-p)\right\rangle & =-\frac{e^{2}}{4 \pi^{2}} \sum_{t= \pm} \int d p_{1} t f\left(E_{p_{1}}-t e \mu_{-}\right), \\
& =-\frac{e^{3}}{4 \pi^{2}} \mu_{-} .
\end{aligned}
$$

Finally using (3.7) to obtain the transport coefficient $\zeta_{1}^{(4)}$, we get

$$
\zeta_{1}^{(4), R}=\tilde{\zeta}_{1}^{(4)}=-\frac{e^{3}}{4 \pi^{2}} \mu_{-} .
$$




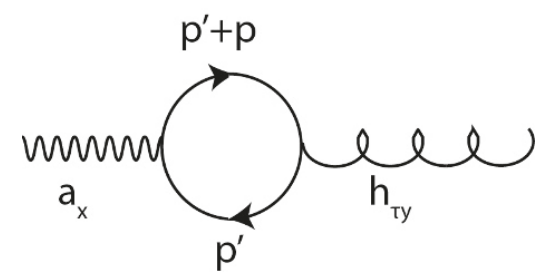

Figure 6. Diagram contributing to $\zeta_{2}^{(4)}$.

Let us now relate this parity odd transport coefficient with the coefficient of the coefficient of the $\mathrm{U}(1)$ anomaly. The anomalous conservation equation for the $\mathrm{U}(1)$ current is given by,

$$
\nabla_{\mu} j^{\mu}=\frac{1}{4} \epsilon^{\mu \nu \rho \lambda}\left[3 c_{A} F_{\mu \nu} F_{\rho \lambda}+c_{m} R_{\beta \mu \nu}^{\alpha} R_{\alpha \rho \lambda}^{\beta}\right] .
$$

The gauge and mixed anomaly coefficients $c_{A}$ and $c_{m}$ for a single Weyl fermion are given by $^{12}$

$$
c_{A}=\frac{e^{3}}{24 \pi^{2}}, \quad c_{m}=\frac{e}{192 \pi^{2}} .
$$

Comparing the coefficient of the anomaly and the transport coefficient, we see that chiral fermions obey the relation

$$
\zeta_{1}^{(4)}=-6 c_{A} \mu_{-} .
$$

This relation obeys the replacement rule relating the gauge anomaly to the transport coefficient $\zeta_{1}^{(4)}$ found in [12]. Alternatively, the coefficients $c_{A}$ and $c_{m}$ can be read out from the anomaly polynomial in four dimensions,

$$
\mathcal{P}_{d=3+1}(\hat{F}, \hat{R})=c_{A} \hat{F} \wedge \hat{F} \wedge \hat{F}+c_{m} \hat{F} \wedge \operatorname{tr}(\hat{R} \wedge \hat{R}) .
$$

Evaluation of $\zeta_{2}^{(4)}$. We first evaluate the relevant two point function in the Euclidean theory, this is given by

$$
\tilde{\zeta}_{2}^{(4)}=\lim _{p_{z} \rightarrow 0} \frac{1}{i p_{z}}\left\langle j^{x}\left(0, p_{z}\right) T^{\tau y}\left(0,-p_{z}\right)\right\rangle .
$$

This correlator is defined by (2.15) and from the expansion of the action in (3.15), we see that it does not involve any contact terms. The expression for the current in momentum space is given by (3.19)) . Converting the stress tensor in (3.18)) to momentum space we obtain

$$
T_{f l}^{\tau y}(-p)=\frac{1}{\beta} \sum_{\omega_{m}} \frac{1}{2} \int \frac{d^{3} p^{\prime}}{(2 \pi)^{3}} \psi_{E}^{\dagger}\left(p^{\prime}+p\right)\left(\gamma^{\tau} i p_{y}^{\prime}+\gamma^{y}\left(i \omega_{m}+e \mu_{-}\right)\right) P_{-} \psi_{E}\left(p^{\prime}\right) .
$$

After performing the Wick contractions we get

$$
\frac{1}{i p_{z}}\left\langle j^{x}\left(0, p_{z}\right) T^{\tau y}\left(0,-p_{z}\right)\right\rangle=
$$

\footnotetext{
${ }^{12}$ For example, see (2.39) of [12].
} 


$$
=\frac{e}{2 \beta p_{z}} \sum_{m} \int \frac{d^{3} p^{\prime}}{(2 \pi)^{3}} \operatorname{Tr}\left[\gamma^{x} S_{E}\left(p^{\prime}+p_{z}\right)\left(\gamma^{\tau} i p^{\prime} y+\gamma^{y}\left(i \omega_{m}+e \mu_{-}\right)\right) S_{E}\left(p^{\prime}\right) P_{+}\right] .
$$

For convenience we break up the terms resulting from the the Wick contractions into two parts, $I_{1}$ and $I_{2}$, which are given by

$$
\begin{aligned}
I_{1}\left(p_{z}\right)= & \frac{e}{i 8 \beta p_{z}} \sum_{m} \int \frac{d^{3} p^{\prime}}{(2 \pi)^{3}} \operatorname{Tr}\left[\gamma^{x} \gamma^{\alpha} \gamma^{\tau} \gamma^{\beta} P_{+}\right] p_{y}^{\prime}\left(\hat{p}^{\prime}+\hat{p}\right)_{t, \alpha}\left(p^{\prime}\right)_{u, \beta} \\
& \times \Delta_{t}\left(i \omega_{m}, p^{\prime}+p\right) \Delta_{u}\left(i \omega_{m}, p^{\prime}\right), \\
= & \frac{i e}{4(2 \pi)^{3}} \sum_{t= \pm} \int \frac{\sin ^{3} \theta}{\cos ^{2} \theta} \cos ^{2} \phi d \theta d \phi \int d p^{\prime} p^{\prime} t f\left(p^{\prime}-t e \mu_{-}\right)+O\left(p_{z}\right), \\
I_{2}\left(p_{z}\right)= & -\frac{e}{8 \beta p_{z}} \sum_{m} \int \frac{d^{3} p^{\prime}}{(2 \pi)^{3}} \operatorname{Tr}\left[\gamma^{x} \gamma^{\alpha} \gamma^{y} \gamma^{\beta} P_{+}\right]\left(i \omega_{m}+e \mu_{-}\right)\left(\hat{p}^{\prime}+\hat{p}\right)_{t, \alpha} \hat{p}_{u, \beta}^{\prime} \\
& \times \Delta_{t}\left(i \omega_{m}, p^{\prime}+p\right) \Delta_{u}\left(i \omega_{m}, p^{\prime}\right), \\
= & \frac{i e}{4(2 \pi)^{3}} \sum_{t= \pm} \int \frac{\sin \theta}{\cos ^{2} \theta} d \theta d \phi \int d p^{\prime} p^{\prime} t f\left(p^{\prime}-t e \mu_{-}\right)+O\left(p_{z}\right) .
\end{aligned}
$$

Here, in the second and the last line of the above equation, we have performed the Matsubara sums, expanded the integrand in the external momenta $p_{z}$ and finally performed the angular integrals using the $i \epsilon$ prescription defined earlier. Note that the angular integrals are considerable simpler than the ones done first in [8] to evaluate this correlator. Using these results, we obtain

$$
\begin{aligned}
\tilde{\zeta}_{2}^{(4)} & =\lim _{p_{z} \rightarrow 0} \frac{1}{i p_{z}}\left\langle j^{x}\left(0, p_{z}\right) T^{\tau y}\left(0,-p_{z}\right)\right\rangle=\lim _{p_{z} \rightarrow 0}\left(I_{1}\left(p_{z}\right)+I_{2}\left(p_{z}\right)\right) \\
& =-\frac{i e}{4 \pi^{2}}\left(\int_{0}^{\infty} d p^{\prime} p^{\prime}\left(f\left(p^{\prime}-e \mu_{-}\right)+f\left(p^{\prime}+e \mu_{-}\right)\right)\right. \\
& =-\frac{i e}{8 \pi^{2}}\left(e^{2} \mu_{-}^{2}+\frac{\pi^{2} T^{2}}{3}\right)
\end{aligned}
$$

We can now relate the above Euclidean correlator, to the desired transport coefficient using (3.7). This results in the following expression

$$
\zeta_{2}^{(4)}=\frac{-e}{8 \pi^{2}}\left(e^{2} \mu_{-}^{2}+\frac{\pi^{2} T^{2}}{3}\right)
$$

Finally let us relate the two terms, that occur in the transport coefficient $\tilde{\zeta}^{(4)}$, to the two anomaly coefficients $c_{A}$ and $c_{m}$, defined in (3.35) and (3.36). First parametrize $\zeta^{(4)}$ following [12] as

$$
\zeta_{2}^{(4)}=-3 c_{A} \mu_{-}^{2}+\tilde{c}_{4 d} T^{2}
$$

Comparing with the results from Kubo formulae (3.45), we obtain

$$
c_{A}=\frac{e^{3}}{24 \pi^{2}}, \quad \tilde{c}_{4 d}=-\frac{e}{24} .
$$




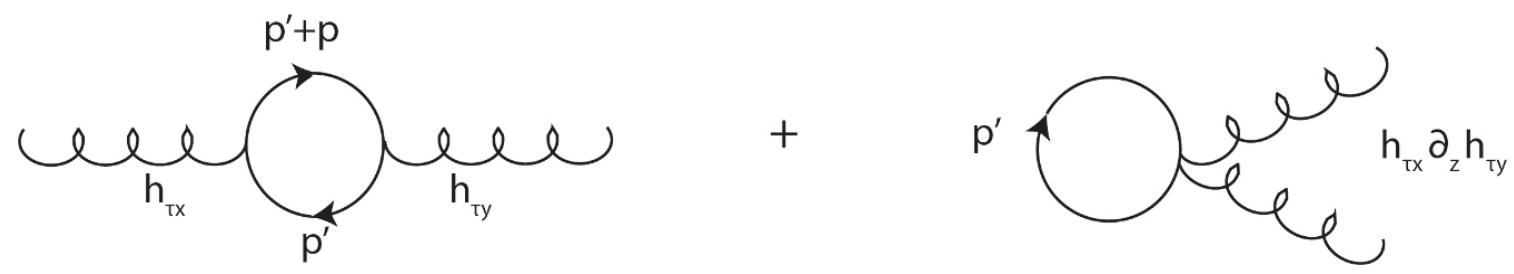

Figure 7. Contributions to $\lambda_{2}^{(4)}$.

From (3.35), (3.36) and (3.38), we see that value of $c_{A}$ obtained using the Kubo formula for $\zeta_{1}^{(4)}$ agree. This is a consistency check on our calculations. Finally we obtain the relation of the

$$
\tilde{c}_{4 d}=-8 \pi^{2} c_{m}
$$

This relation was obtained using consistency of the Euclidean vacuum in in [12]. This direct perturbative calculation provides a check on this argument. Note that the transport coefficient $\zeta_{2}^{(4)}$ has been evaluated earlier by [8], here we have demonstrated that the angular integrals simplify considerably on taking the zero momentum limit first. In fact doing this leads directly to the moments of the Fermi-Dirac distribution.

Evaluation of $\lambda_{2}^{(4)}$. The last transport coefficient of interest in 4 dimensions is $\lambda_{2}^{(4)}$. The corresponding coefficient in the Euclidean theory is given by the following two point function

$$
\tilde{\lambda}_{2}^{(4)}=\lim _{p_{z} \rightarrow 0} \frac{1}{i p_{z}}\left\langle T^{\tau x}\left(0, p_{z}\right) T^{\tau y}\left(0, p_{z}\right)\right\rangle
$$

From (2.16) and the perturbative expansion of the action in (3.15), we see that there is a contact term due to $S^{(2)}$ from the action. Therefore we obtain

$$
\begin{aligned}
\tilde{\lambda}_{2}^{(4)} & =A+B, \\
A & =\lim _{p \rightarrow 0, p_{0} \rightarrow 0} \frac{1}{i p_{z}}\left\langle T_{f l}^{\tau x}\left(0, p_{z}\right) T_{f l}^{\tau y}\left(0,-p_{z}\right)\right\rangle_{E}, \\
B & =\lim _{p_{z} \rightarrow 0} \frac{1}{i p_{z}}\left(\frac{-i p_{z}}{8 \beta}\right) \sum_{m}\left\langle\int \frac{d^{3} p^{\prime}}{(2 \pi)^{3}} \psi_{E}^{\dagger}\left(\omega_{m}, p^{\prime}\right) \Gamma^{z x y} P_{-} \psi_{E}\left(\omega_{m}, p^{\prime}\right)\right\rangle .
\end{aligned}
$$

The expression for the stress tensors in momentum space can be read out from the following expressions

$$
\begin{aligned}
T_{f l}^{\tau x}(p) & =\frac{1}{2 \beta} \sum_{m} \int \frac{d^{3} p^{\prime}}{(2 \pi)^{3}} \psi_{E}^{\dagger}\left(p^{\prime}-p\right)\left[\gamma^{\tau} i p_{x}^{\prime}+\gamma^{x}\left(i \omega+e \mu_{-}\right)\right] P_{-} \psi_{E}\left(p^{\prime}\right), \\
T_{f l}^{\tau y}(-p) & =\frac{1}{2 \beta} \sum_{m} \int \frac{d^{3} p^{\prime}}{(2 \pi)^{3}} \psi_{E}^{\dagger}\left(p^{\prime}+p\right)\left[\gamma^{\tau} i p_{y}^{\prime}+\gamma^{y}\left(i \omega_{m}+e \mu_{-}\right)\right] P_{-} \psi_{E}\left(p^{\prime}\right) .
\end{aligned}
$$

This contribution from the contact term $B$ in (3.50) was absent in the calculations of [10] and was considered in the recent work of [22]. We will see that only after this contact term is considered, we will obtain the result consistent with the replacement rule. Performing the Wick contractions and the Matsubara sums, the term $A$ reduces to

$$
A=\lim _{p_{z} \rightarrow 0} \frac{\left\langle T^{\tau x}\left(0, p_{z}\right) T^{\tau y}\left(0,-p_{z}\right)\right\rangle}{i p_{z}},
$$




$$
=\frac{1}{16 \pi^{2}}\left(\left(e \mu_{-}\right)^{3}+\pi^{2} T^{2} \mu_{-}\right) .
$$

We now need to compute the contribution due to the contact term. This is given by

$$
\begin{aligned}
B & =\frac{i}{\beta} \sum_{m, t= \pm} \frac{1}{8} \int \frac{d^{3} p^{\prime}}{(2 \pi)^{3}} \operatorname{Tr}\left[\gamma^{\alpha} \gamma^{z} \gamma^{x} \gamma^{y} P_{-}\right]\left(\frac{1}{2} \hat{p}_{t, \alpha}^{\prime} \Delta_{t}\left(i \omega_{m}, p^{\prime}\right)\right) \\
& =\frac{1}{8} \sum_{t= \pm} \int \frac{d^{3} p^{\prime}}{(2 \pi)^{3}} t f\left(E_{p^{\prime}}-t e \mu_{-}\right) \\
& =\frac{1}{16 \pi^{2}} \int d p^{\prime} p^{\prime 2}\left(f\left(E_{p_{1}}-e \mu_{-}\right)-f\left(E_{p_{1}}+e \mu_{-}\right)\right) \\
& =\frac{1}{48 \pi^{2}}\left(\left(e \mu_{-}\right)^{3}+\pi^{2} T^{2} \mu_{-}\right)
\end{aligned}
$$

Combining the results of term $A$ and $B$, from (3.52) and (3.53), we get

$$
\tilde{\lambda}_{2}^{(4)}=\frac{1}{12 \pi^{2}}\left(\left(e \mu_{-}\right)^{3}+e \pi^{2} T^{2} \mu_{-}\right) .
$$

We can now use the relation (3.7) to obtain the transport result of interest

$$
\begin{aligned}
\lambda_{2}^{(4)} & =-\tilde{\lambda}_{2}^{(4)}, \\
& =\frac{-1}{12 \pi^{2}}\left(\left(e \mu_{-}\right)^{3}+e \pi^{2} T^{2} \mu_{-}\right) .
\end{aligned}
$$

Let us now verify that this result is consistent with the replacement rule. Following [12], we parametrize transport coefficient as

$$
\lambda_{2}^{(4)}=2\left(-c_{A} \mu_{-}^{3}+\tilde{c}_{4 d} T^{2} \mu_{-}\right) .
$$

Comparing this parametrization with (3.55), we obtain

$$
c_{A}=\frac{e^{3}}{24 \pi^{2}}, \quad \tilde{c}_{4 d}=-\frac{e}{24} .
$$

From (3.36), we see that the value of $c_{A}$ is that of a single Weyl fermion. Furthermore $\tilde{c}_{4 d}$ is identical to that obtained evaluating $\zeta_{1}^{(4)}$ in (3.47) and it also satisfies the relation

$$
\tilde{c}_{4 d}=-8 \pi^{2} c_{m}
$$

This relation was found by the argument involving the consistency of the Euclidean vacuum in [12]. It is important to note that the contribution of the contact term $B$ was important to arrive at these results.

The transport coefficients involving chiral fermions in $d=4$ are summarized in the table 1. We note that in [19], the contribution of chiral gravitinos to these transport coefficients was discussed. However gravitinos cannot be charged in flat space without violating the gauge symmetry, $\psi_{\mu} \rightarrow \psi_{\mu}+\partial_{\mu} \epsilon$. Therefore they do not admit any U(1) charge current which implies there is no contribution to these transport coefficients from chiral gravitinos. ${ }^{13}$

\footnotetext{
${ }^{13}$ We thank Zohar Komargodski for emphasizing this to us.
} 


\begin{tabular}{|c|c|c|}
\hline Dimension & Correlator & Value \\
\hline $\mathrm{d}=4$ & $\zeta_{1}^{(4)}$ & $\frac{-e^{3}}{4 \pi^{2}} \mu_{-}$ \\
\hline & $\zeta_{2}^{(4)}$ & $\frac{-e}{8 \pi^{2}}\left(e^{2} \mu_{-}^{2}+\frac{\pi^{2} T^{2}}{3}\right)$ \\
\hline & $\lambda_{2}^{(4)}$ & $-\frac{1}{12 \pi^{2}}\left(\left(e \mu_{-}\right)^{3}+e \pi^{2} T^{2} \mu_{-}\right)$ \\
\hline
\end{tabular}

Table 1. Transport coefficients in 4 dimensions.

\section{Anomalies and transport in $d=6$}

In this section we evaluate all the parity odd transport coefficients occurring in $d=6$, at the leading order in derivatives, using the same methods developed in the previous section. This analysis will serve as a check of the computation in [22], of transport coefficients determined only by the charge current gauge anomaly. This was done using different approach than the one adopted in this paper. Our analysis will also use the $i \epsilon$ prescription, developed in the previous section, to perform the angular integrals. Therefore the results of the $d=6$ calculations also test this prescription. Finally we also determine the contributions to the transport coefficients from the mixed as well as the pure gravitational anomaly, which was not done in [22]. Our results are in agreement with the replacement rule for the mixed and pure gravitational anomaly, found by $[7,12]$ for chiral fermions. But for the case of chiral gravitinos which contribute to the pure gravitational anomaly, we see that the their contribution to the transport coefficients does not obey the replacement rule. We precisely identify the terms which contribute to the anomaly polynomial but not to the transport coefficient.

We begin with parametrization of the constitutive relations in $d=6$, where we focus on the the leading parity odd transport coefficients. Following the parametrization given in [22], the charge current and the stress tensor is given by

$$
\begin{aligned}
j^{\mu}= & n u^{\mu}+\frac{\zeta_{1}^{(6)}}{3} \epsilon^{\mu \nu \alpha \beta \gamma \delta} u_{\nu} F_{\alpha \beta} F_{\gamma \delta} \\
& +\frac{\zeta_{2}^{(6)}}{3} \epsilon^{\mu \nu \alpha \beta \gamma \delta} u_{\nu} \partial_{\alpha} u_{\beta} F_{\gamma \delta}+\frac{\zeta_{3}^{(6)}}{3} \epsilon^{\mu \nu \alpha \beta \gamma \delta} u_{\nu} \partial_{\alpha} u_{\beta} \partial_{\gamma} u_{\delta}, \\
T^{\mu \nu}= & (\epsilon+p) u^{\mu} u^{\nu}-p g^{\mu \nu}+u^{\mu}\left(\frac{\lambda_{1}^{(6)}}{3} \epsilon^{\nu \rho \alpha \beta \gamma \delta} u_{\rho} F_{\alpha \beta} F_{\gamma \delta}\right. \\
& \left.+\frac{\lambda_{2}^{(6)}}{3} \epsilon^{\nu \rho \alpha \beta \gamma \delta} u_{\rho} \partial_{\alpha} u_{\beta} F_{\gamma \delta}+\frac{\lambda_{3}^{(6)}}{3} \epsilon^{\nu \rho \alpha \beta \gamma \delta} u_{\rho} \partial_{\alpha} u_{\beta} \partial_{\gamma} u_{\delta}\right)+(\mu \rightarrow \nu),
\end{aligned}
$$

where, $\epsilon, p, n, \mu$ are the thermodynamic variables denoting energy density, pressure and charge density. The six anomalous transport coefficients are denoted by $\lambda_{1}^{(6)}, \lambda_{2}^{(6)}, \lambda_{3}^{(6)}, \zeta_{1}^{(6)}, \zeta_{2}^{(6)}, \zeta_{3}^{(6)}$. The Minkowski metric, we work in, has the signature $\left(\eta_{\mu \nu}\right)=$ $\operatorname{diag}(1,-1,-1,-1,-1,-1)$. Let us also label the directions as follows, the position vector or momentum vector has components in directions labeled by $(t, a, z, x, y, b)$. We choose the orientation in $d=6$ so that $\epsilon^{t a z x y b}=1$. To arrive at the Kubo formula, we assume that the fluid is perturbed from rest and its velocity field is given by $\left.u^{\mu}=\left(1,0, v^{z}, v^{x}, 0,0\right)\right)$. 
The velocity field $v^{z}(t, b)$ depends only on time $t$ and coordinate $b$, while the velocity field $v^{x}(t, z)$ depends only on time and the coordinate $z$. We then consider the metric perturbation $h_{t z}(t, b)$ and $h_{t x}(t, y)$, while the rest of the fluctuations are set to zero. Similarly we consider gauge fluctuations $a_{z}(t, b)$ and $a_{x}(t, y)$. We summarize the perturbations and the coordinates they depend on in the following equations

$$
\begin{aligned}
u^{\mu} & =\left(1,0, v^{z}(t, b), v^{x}(t, z), 0,0\right), \\
u_{\mu} & =\left(1,0,-v^{z}(t, b)+h_{t z}(t, b),-v^{x}(t, z)+h_{t x}(t, z), 0,0\right), \\
A_{\mu} & =\left(\mu, 0, a_{z}(t, b), a_{x}(t, y), 0,0\right), \\
g_{\mu \nu} & =\eta_{\mu \nu}+h_{\mu \nu}, \quad \text { with } h_{t z}(t, b), h_{t x}(t, y) \neq 0 .
\end{aligned}
$$

With this choice of the polarizations and perturbations, it can be easily seen that the contributions proportional to the anomalous transport coefficients in (4.1), appear at second order in fluctuations and derivatives. Therefore the Kubo formulae for these transport coefficients are given by three point functions. Expanding the constitutive relations to second order, we obtain following expressions relating the three point functions to the transport coefficients

$$
\begin{aligned}
& \frac{8}{3} \zeta_{1}^{(6)}=-\lim _{k_{y}, p_{b} \rightarrow 0} \frac{\left\langle j^{a}(p+k) j^{x}(-k) j^{z}(-p)\right\rangle}{i k_{y} i p_{b}}, \\
& \frac{2}{3} \zeta_{2}^{(6)}=-\frac{\left\langle j^{a}(p+k) j^{x}(-k) T^{t z}(-p)\right\rangle}{i k_{y} i p_{b}}, \\
& \frac{2}{3} \zeta_{3}^{(6)}=-\frac{\left\langle j^{a}(k+p) T^{t x}(-k) T^{t z}(-p)\right\rangle}{i k_{y} i p_{b}}, \\
& \frac{8}{3} \lambda_{1}^{(6)}=-\lim _{k_{y}, p_{b} \rightarrow 0} \frac{\left\langle j^{a}(p+k) j^{x}(-k) T^{t z}(-p)\right\rangle}{i k_{y} i p_{b}}, \\
& \frac{2}{3} \lambda_{2}^{(6)}=-\lim _{k_{y}, p_{b} \rightarrow 0} \frac{\left\langle j^{a}(p+k) T^{t x}(-k) T^{t z}(-p)\right\rangle}{i k_{y} i p_{b}} \\
& \frac{2}{3} \lambda_{3}^{(6)}=-\lim _{k_{y}, p_{b} \rightarrow 0} \frac{\left\langle T^{t a}(p+k) T^{t x}(-k) T^{t z}(-p)\right\rangle}{i k_{y} i p_{b}} .
\end{aligned}
$$

To un-clutter our notations, it is understood that the external frequencies are set to zero from the beginning and that the only non-zero component of the momentum vectors $p, k$ are in the $b$ and the $y$ directions respectively. From (4.3), we arrive at the following relations between the transport coefficients

$$
4 \lambda_{1}^{(6)}=\zeta_{2}^{(6)}, \quad \lambda_{2}^{(6)}=\zeta_{3}^{(6)} .
$$

Therefore it is sufficient to focus on evaluating the coefficients $\zeta_{1}^{(6)}, \zeta_{2}^{(6)}, \zeta_{3}^{(6)}$ and $\lambda_{3}^{(6)}$. The strategy for evaluating the correlators will be same, we will first evaluate them in the Euclidean theory and then analytically continue them to the Lorentzian theory. Let the value of the corresponding correlators in the Euclidean theory by $\tilde{\zeta}_{1}^{(6)}, \tilde{\zeta}_{2}^{(6)}, \tilde{\zeta}_{3}^{(6)}$ and $\tilde{\lambda}_{3}^{(6)}$. Then going carrying out the analytic continuation leads to the following relations

$$
\tilde{\zeta}_{1}^{(6)}=\zeta_{1}^{(6)}, \quad \tilde{\zeta}_{2}^{(6)}=i \zeta_{2}^{(6)},
$$




$$
\tilde{\zeta}_{3}^{(6)}=-\zeta_{3}^{(6)}, \quad \tilde{\lambda}_{3}^{(6)}=-i \lambda_{3}^{(6)}
$$

We will now proceed to evaluation of the contribution of these correlators, first for chiral fermions and then the contribution of the chiral gravitinos to $\lambda_{3}^{(6)}$.

\subsection{Chiral fermions}

For Weyl fermions in $d=6$, the partition function is given by

$$
\mathcal{Z}=\int \mathcal{D} \psi^{\dagger} \mathcal{D} \psi \exp \left(S_{E}\right)
$$

where $\psi$ is a Euclidean Dirac spinor in $d=6$ and the Euclidean action is given by

$$
S_{E}=\int d \tau d^{5} x \sqrt{g} e_{a}^{\mu} \psi^{\dagger} \gamma^{a} D_{\mu} P_{-} \psi
$$

The covariant derivative is defined as

$$
D_{\rho} \psi=\partial_{\rho} \psi+\frac{1}{2} \omega_{\rho a b} \sigma^{a b} \psi+i e A_{\rho} \psi
$$

where, $\omega_{\rho a b}$ is the spin connection and $\sigma^{a b}=\frac{1}{4}\left[\gamma_{a}, \gamma_{b}\right]$. The gamma matrices follow the same conventions as before. The conventions for the metric, vierbein and their perturbations remain same as that in $d=4$ which is given in equations (3.11), (3.13) and (3.14). The chiral projection operator is defined by

$$
P_{ \pm}=\frac{1}{2}\left(1 \pm \gamma^{7}\right), \quad \quad \gamma^{7}=i \gamma^{\tau} \gamma^{a} \gamma^{z} \gamma^{x} \gamma^{y} \gamma^{b}
$$

To evaluate the Feynman diagrams, we will need the following trace

$$
\operatorname{Tr}\left(\gamma^{p} \gamma^{q} \gamma^{r} \gamma^{s} \gamma^{t} \gamma^{u} \gamma^{7}\right)=-8 \epsilon^{p q r s t u}, \quad \quad \epsilon^{\tau a z x y b}=i .
$$

Wick contractions are performed by the propagator given in (3.21). Expanding the action in in these fluctuations, we obtain

$$
\begin{aligned}
S_{E}^{(0)}= & \int d \tau d^{5} x \psi^{\dagger}\left(\gamma^{\tau} D_{\tau}+\gamma^{i} \partial_{i}\right) P_{-} \psi \\
S_{E}^{(1)}= & -\int d \tau d^{5} x \frac{h_{\mu \nu}}{4} \psi^{\dagger}\left(\gamma^{\mu} \partial^{\nu}+\gamma^{\nu} \partial^{\mu}\right) P_{-} \psi \\
& +i e \int d \tau d^{5} x \psi^{\dagger} \gamma^{\mu} A_{\mu} \psi+O(h a)+O\left(h^{2}, a\right)+O\left(h^{3}\right), \\
S_{E}^{(2)}= & \int d \tau d^{5} x \frac{h_{\lambda \alpha} \partial_{\mu} h_{\nu \alpha}}{16} \psi^{\dagger} \Gamma^{\mu \lambda \nu} P_{-} \psi+O\left(h^{3}\right) .
\end{aligned}
$$

At first glance one would have expected that we must expand the action to at least $O\left(h^{3}\right), O\left(h^{2} a\right)$ to obtain all contributions to the correlators of interest. This is because now we are evaluating three point functions. However terms involving $O(h, a), O\left(h^{3}\right), O\left(h^{2}, a\right)$ in the expansion $S_{E}^{(1)}$ of (4.11), do not have sufficient number of $\Gamma$ matrices to ensure a non-zero result for the correlators of interest. We need $6 \Gamma$ matrices to ensure non-zero 
result in $d=6$. There is another reason the $O(h A)$ terms do not contribute. From the structure of the perturbations in (4.2) of the metric and the gauge field, it is easy to see there are no terms of the type $O\left(h_{\tau x} a_{z}\right), O\left(h_{\tau z} a_{x}\right)$ and therefore there is no contributions to the correlators of interest. Finally the reason $O\left(h^{3}\right)$ term is not considered in $S_{E}^{(2)}$ of (4.11) is again due to the fact that such terms do not have sufficient number of gamma matrices to saturate the correlator of interest. Therefore the terms in the expansion (4.11) is sufficient for evaluating all the transport coefficients of interest.

Evaluation of $\zeta_{1}^{(6)}$. We begin by evaluating the coefficient $\zeta_{1}^{(6)}$, the corresponding correlator is given by

$$
\begin{aligned}
\left\langle j^{\mu} j^{\nu} j^{\rho}\right\rangle= & -\frac{1}{\mathcal{Z}_{E}^{(0)}} \int \mathcal{D} \psi^{\dagger} \mathcal{D} \psi e^{S_{E}^{(0)}}\left(\frac{\delta S_{E}}{\sqrt{g} \delta A_{\mu}} \frac{\delta S_{E}}{\sqrt{g} \delta A_{\nu}} \frac{\delta S_{E}}{\sqrt{g} \delta A_{\rho}}\right. \\
& +\frac{\delta^{2} S_{E}}{\sqrt{g} \delta A_{\mu} \sqrt{g} \delta A_{\nu}} \frac{\delta S_{E}}{\sqrt{g} \delta A_{\rho}}+\frac{\delta^{2} S_{E}}{\sqrt{g} \delta A_{\mu} \sqrt{g} \delta A_{\rho}} \frac{\delta S_{E}}{\sqrt{g} \delta A_{\nu}} \\
& \left.+\frac{\delta S_{E}}{\sqrt{g} \delta A_{\mu}} \frac{\delta^{2} S_{E}}{\sqrt{g} \delta A_{\nu} \sqrt{g} \delta A_{\rho}}+\frac{\delta^{3} S_{E}}{\sqrt{g} \delta A_{\mu} \sqrt{g} \delta A_{\nu} \sqrt{g} \delta A_{\rho}}\right) .
\end{aligned}
$$

Here it is understood that only the connected correlators should be evaluated while performing Wick contractions. The correlator of interest is given by

$$
\tilde{\zeta}_{1}^{(6)}=-\lim _{p_{b}, k_{y} \rightarrow 0} \frac{3}{8} \frac{\left\langle j^{a}(k+p) j^{x}(-k) j^{z}(-p)\right\rangle}{i p_{b} i k_{y}} .
$$

As we have mentioned earlier, we choose the kinematic configurations in which the momentum vectors $p, k$ have components only in the $b, y$ directions. The frequency dependence of the external momenta has been set to zero from the start. First note that from the expansion of the action in (4.11), the gauge fluctuations appear linearly and therefore there are no contributions from terms involving 2 derivatives of the action in (4.12). Therefore the correlator in (4.13) is obtained by performing Wick contractions of the flat space charge current which is given by

$$
j_{f l}^{\mu}(p)=-i e \frac{1}{\beta} \sum_{m} \int \frac{d^{5} p^{\prime}}{(2 \pi)^{5}} \psi^{\dagger}\left(\omega_{m}, p^{\prime}-p\right) \gamma^{\mu} P_{-} \psi\left(\omega_{m}, p^{\prime}\right)
$$

After performing the two possible Wick contractions, we obtain

$$
\begin{aligned}
\left\langle j^{a}(p+k) j^{x}(-k) j^{z}(-p)\right\rangle & =-\frac{i e^{3}}{\beta} \sum_{m} \int \frac{d^{5} p_{1}}{(2 \pi)^{5}} \\
\times & \left(\operatorname{Tr}\left[\gamma^{a} S\left(\omega_{m}, p^{\prime}+k+p\right) \gamma^{x} S\left(\omega_{m}, p^{\prime}+p\right) \gamma^{z} S\left(\omega_{m}, p\right) P_{+}\right]\right. \\
+ & \left.\operatorname{Tr}\left[\gamma^{z} S\left(\omega_{m}, p^{\prime}-p\right) \gamma^{x} S\left(\omega_{m}, p^{\prime}-p-k\right) \gamma^{a} S_{E}\left(\omega_{m}, p^{\prime}\right) P_{+}\right]\right) .
\end{aligned}
$$

The two Wick contractions are summarized in the figure 8. The details of carrying out the Matsubara sums and then the angular integrals using the $i \epsilon$ prescription is given in the appendix B.1. The end result for the Euclidean coefficient is

$$
\tilde{\zeta}_{1}^{(6)}=\frac{3 e^{4}}{64 \pi^{3}} \int_{0}^{\infty}\left(f\left(p-e \mu_{-}\right)-f\left(p+e \mu_{-}\right)\right)
$$




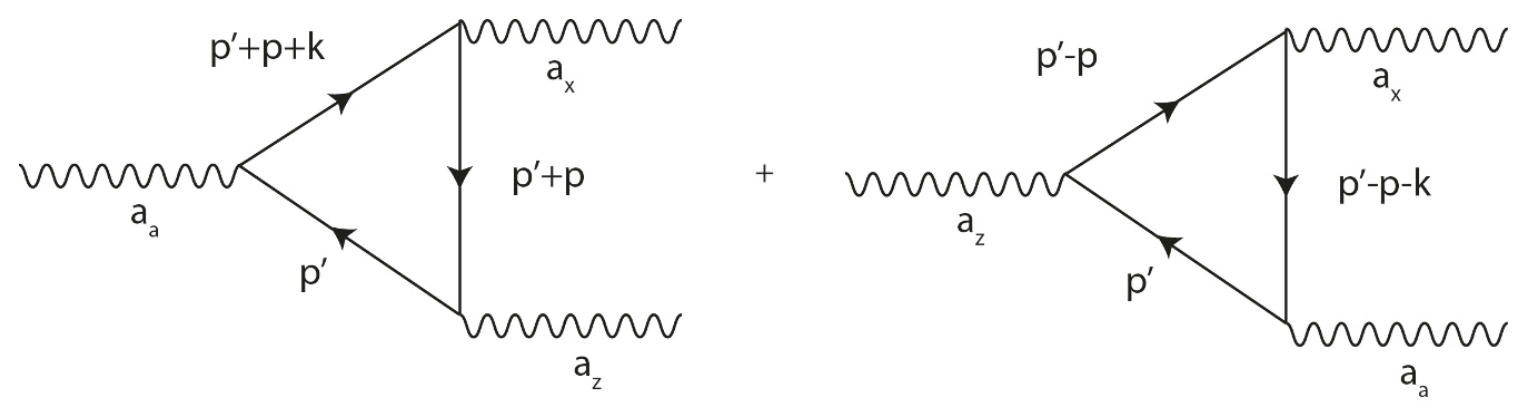

Figure 8. Diagrams contributing to $\zeta_{1}^{(6)}$.

$$
=\frac{3 e^{4}}{64 \pi^{3}} \mu_{-}
$$

Using the relations for analytic continuation given in (4.5), we obtain

$$
\zeta_{1}^{(6)}=\frac{3 e^{4}}{64 \pi^{3}} \mu_{-}
$$

Let us now compare these coefficients to that in the anomaly polynomial in $d=6$. The anomaly polynomial for a single Weyl fermions in $d=6$ is given by [19]

$$
\mathcal{P}_{d=5+1}(\hat{F}, \hat{R})=c_{\alpha} \hat{F}^{4}+c_{\beta} F^{2} \operatorname{Tr}\left(\hat{R}^{2}\right)+c_{\gamma}\left(\operatorname{Tr}\left(\hat{R}^{2}\right)\right)^{2}+c_{\delta}\left(\frac{1}{4} \operatorname{Tr}\left(\hat{R}^{4}\right)-\frac{1}{8} \operatorname{Tr}\left(\hat{R}^{2}\right)^{2}\right),
$$

where

$$
\begin{aligned}
c_{\alpha} & =-\frac{e^{4}}{192 \pi^{3}}, & c_{\beta} & =-\frac{e^{2}}{768 \pi^{3}}, \\
c_{\gamma} & =-\frac{7}{184320 \pi^{3}}, & c_{\delta} & =-\frac{1}{11520 \pi^{3}} .
\end{aligned}
$$

Thus the transport coefficient satisfies the relation

$$
\zeta_{1}^{(6)}=-9 c_{\alpha} \mu_{-}
$$

This relation is the expected relation between the gauge anomaly coefficient and the transport coefficient of interest, predicted by general considerations of hydrodynamics in [5, 12].

Evaluation of $\zeta_{\mathbf{2}}^{(\mathbf{6})}$. The correlator of interest for the transport coefficient $\zeta_{2}^{(6)}$ is given by

$$
\begin{aligned}
\left\langle j^{\mu} j^{\nu} T^{\rho \sigma}\right\rangle=- & \frac{2}{\mathcal{Z}_{E}^{(0)}} \int \mathcal{D} \psi^{\dagger} \mathcal{D} \psi e^{S_{E}^{(0)}} \times \\
& \times\left(\frac{\delta S_{E}}{\delta \sqrt{g} A_{\mu}} \frac{\delta S_{E}}{\sqrt{g} \delta A_{\nu}} \frac{\delta S_{E}}{\sqrt{g} \delta g_{\rho \sigma}}+\frac{\delta^{2} S_{E}}{\sqrt{g} \delta A_{\mu} \sqrt{g} \delta A_{\nu}} \frac{\delta S_{E}}{\sqrt{g} \delta g_{\rho \sigma}}\right. \\
& +\frac{\delta^{2} S_{E}}{\sqrt{g} \delta A_{\mu} \sqrt{g} \delta g_{\rho \sigma}} \frac{\delta S_{E}}{\sqrt{g} \delta A_{\nu}}+\frac{\delta S_{E}}{\sqrt{g} \delta A_{\mu}} \frac{\delta^{2} S_{E}}{\sqrt{g} \delta A_{\nu} \delta \sqrt{g} g_{\rho \sigma}} \\
& \left.+\frac{\delta^{3} S_{E}}{\sqrt{g} \delta a_{\mu} \sqrt{g} \delta A_{\nu} \sqrt{g} \delta g_{\rho \sigma}}\right) \cdot
\end{aligned}
$$




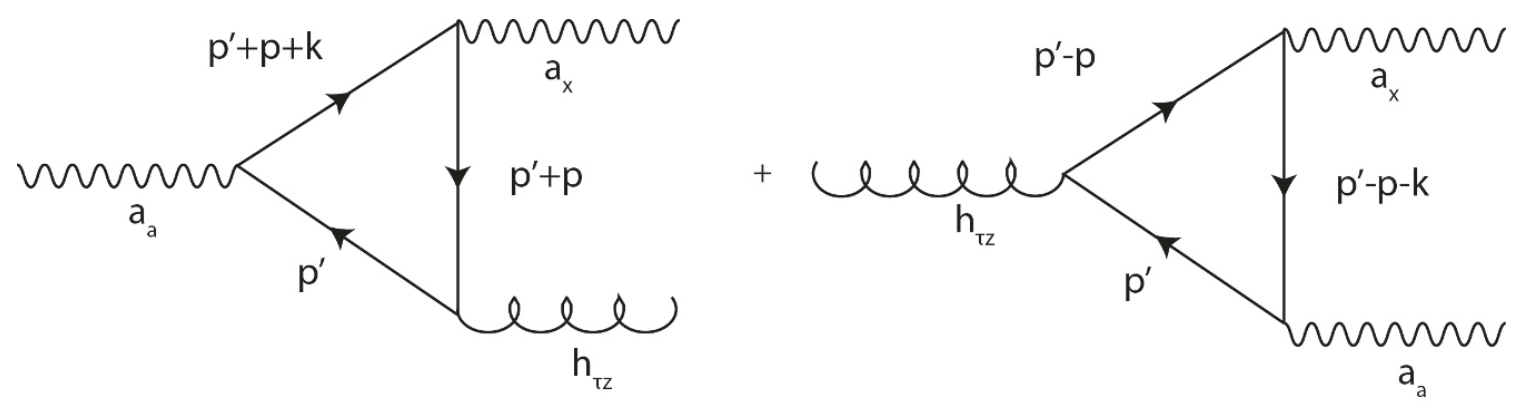

Figure 9. Contributions to $\zeta_{2}^{(6)}$.

Here again it is understood that only connected Wick contractions need to be evaluated. After evaluating the correlator in Euclidean space, we extract the following coefficient

$$
\tilde{\zeta}_{2}^{(6)}=-\lim _{p_{b}, k_{y} \rightarrow 0} \frac{3}{2} \frac{\left\langle j^{a}(k+p) j^{x}(-k) T^{\tau z}(-p)\right\rangle}{i p_{b} i k_{y}} .
$$

From the expansion of the action in (4.11), we see that none of the contact terms contribute to this correlator and therefore we can evaluate the correlator in (4.22), by performing Wick contractions on the flat space charge current given in (4.14) as well as the stress tensor given by

$$
T_{f l}^{\tau z}(-p)=\frac{1}{\beta} \sum_{m} \int \frac{d^{5} p^{\prime}}{(2 \pi)^{5}} \psi^{\dagger}\left(p^{\prime}+p\right)\left(\gamma^{\tau} i p_{z}^{\prime}+\gamma^{z}\left(i \omega_{m}+\mu_{-}\right)\right) P_{-} \psi\left(p^{\prime}\right) .
$$

The two possible Wick contractions are summarized in the figure 9. The details of the Wick contractions, Matsubara sums and angular integrals resulting in the calculation are provided in B.2. The end result of the corresponding Euclidean transport coefficient is given by

$$
\begin{aligned}
\tilde{\zeta}_{2}^{(6)} & =i \frac{3 e^{2}}{16 \pi^{3}} \int_{0}^{\infty} p\left(f\left(p-e \mu_{-}\right)-f\left(p+e \mu_{-}\right),\right. \\
& =i \frac{3 e^{2}}{32 \pi^{3}}\left(e^{2} \mu_{-}^{2}+\frac{\pi^{2} T^{2}}{3}\right) .
\end{aligned}
$$

Again using the relations in (4.5), we obtain the transport coefficient of interest to be

$$
\zeta_{2}^{(6)}=\frac{3 e^{2}}{32 \pi^{3}}\left(e^{2} \mu_{-}^{2}+\frac{\pi^{2} T^{2}}{3}\right) .
$$

Let us compare this coefficient with the coefficients in the anomaly polynomial. For this, following [12], we first parametrize the transport coefficient as

$$
\zeta_{2}^{(6)}=3\left(-6 c_{\alpha} \mu_{-}^{3}+\tilde{c}_{m}^{6 d} T^{2}\right),
$$

where $c_{\alpha}$ for Weyl fermions is read out from (4.18). Comparing (4.25), we see that $\tilde{c}_{m}^{6 d}$ is related to the mixed gravitational anomaly coefficient $c_{\beta}$ by

$$
\tilde{c}_{m}^{6 d}=-8 \pi^{2} c_{\beta} .
$$

In [12], such a relation was obtained using the argument involving the consistency of the Euclidean vacuum. Here we have explicitly demonstrated this relation using the perturbative computation. 
Evaluation of $\zeta_{3}^{(6)}$. Now consider the correlator

$$
\begin{aligned}
\left\langle j^{\mu} T^{\nu \beta} T^{\rho \sigma}\right\rangle=- & \frac{4}{\mathcal{Z}_{E}^{(0)}} \int \mathcal{D} \psi^{\dagger} \mathcal{D} \psi e^{S_{E}^{(0)}} \times \\
\times & \left(\frac{\delta S_{E}}{\sqrt{g} \delta A_{\mu}} \frac{\delta S_{E}}{\sqrt{g} \delta g_{\nu \beta}} \frac{\delta S_{E}}{\sqrt{g} \delta g_{\rho \sigma}}+\frac{\delta^{2} S_{E}}{\sqrt{g} \delta A_{\mu} \sqrt{g} \delta g_{\nu \beta}} \frac{\delta S_{E}}{\sqrt{g} \delta g_{\rho \sigma}}\right. \\
& +\frac{\delta^{2} S_{E}}{\sqrt{g} \delta A_{\mu} \sqrt{g} \delta g_{\rho \sigma}} \frac{\delta S_{E}}{\sqrt{g} \delta g_{\nu \beta}}+\frac{\delta S_{E}}{\sqrt{g} \delta A_{\mu}} \frac{\delta^{2} S_{E}}{\sqrt{g} \delta g_{\nu \beta} \sqrt{g} \delta g_{\rho \sigma}} \\
& \left.+\frac{\delta^{3} S_{E}}{\sqrt{g} \delta A_{\mu} \sqrt{g} \delta g_{\nu \beta} \sqrt{g} \delta g_{\rho \sigma}}\right) .
\end{aligned}
$$

The corresponding transport coefficient in the Euclidean theory is given by

$$
\tilde{\zeta}_{3}^{(6)}=-\lim _{p_{b}, k_{y} \rightarrow 0} \frac{3}{2} \frac{\left\langle j^{a}(k+p) T^{\tau x}(-k) T^{\tau z}(-p)\right\rangle}{i p_{b} i k_{y}} .
$$

Now from examining the expansion of the action in (4.11), we see that the only non-zero contributions to the correlator are given by

$$
\begin{aligned}
\left\langle j^{\mu} T^{\nu \beta} T^{\rho \sigma}\right\rangle & =A+B \\
A & =-\frac{4}{\mathcal{Z}_{E}^{(0)}} \int \mathcal{D} \psi^{\dagger} \mathcal{D} \psi e^{S_{E}^{(0)}} \frac{\delta S_{E}}{\sqrt{g} \delta A_{\mu}} \frac{\delta S_{E}}{\sqrt{g} \delta g_{\nu \beta}} \frac{\delta S_{E}}{\sqrt{g} \delta g_{\rho \sigma}} \\
B & =-\frac{4}{\mathcal{Z}_{E}^{(0)}} \int \mathcal{D} \psi^{\dagger} \mathcal{D} \psi e^{S_{E}^{(0)}} \frac{\delta S_{E}}{\sqrt{g} \delta A_{\mu}} \frac{\delta^{2} S_{E}}{\sqrt{g} \delta g_{\nu \beta} \sqrt{g} \delta g_{\rho \sigma}} .
\end{aligned}
$$

Therefore we have a contribution from the Wick contractions of the flat space currents denoted by $A$, together with a contact term which involves Wick contraction with a flat space current denoted by $B$. These are summarized in figure 10 and figure 11 respectively. We have evaluated these contributions in detail in appendix B.3. The end result for the Euclidean correlator is given by

$$
\begin{aligned}
\tilde{\zeta}_{3}^{(6)} & =\frac{-3 e}{16 \pi^{3}} \int_{0}^{\infty} d p p^{2}\left(f\left(p_{1}-e \mu_{-}\right)-f\left(p+e \mu_{-}\right)\right), \\
& =\frac{-e}{16 \pi^{3}}\left[\left(e \mu_{-}\right)^{3}+e \pi^{2} T^{2} \mu_{-}\right] .
\end{aligned}
$$

Again using the relations in (4.5) to go from the Euclidean correlator to the transport coefficient of interest in the Lorentzian theory, we obtain

$$
\zeta_{3}^{(6), R}=\frac{e}{16 \pi^{3}}\left[\left(e \mu_{-}\right)^{3}+e \pi^{2} T^{2} \mu_{-}\right]
$$

Let us compare this transport coefficient to what is expected from the replacement rule. Following [12], we parametrize the coefficient as

$$
\zeta_{3}^{(6), R}=6\left(-2 c_{\alpha} \mu_{-}^{3}+\tilde{c}_{m}^{6 d} T^{2} \mu_{-}\right)
$$



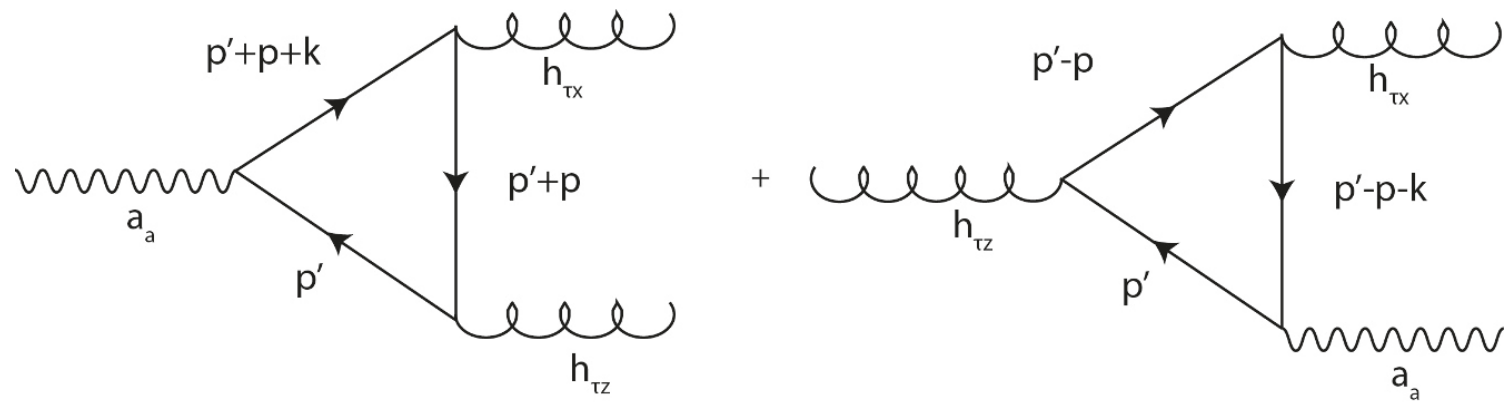

Figure 10. Contributions to $\zeta_{3}^{(6)}$ from Wick contractions of flat currents.

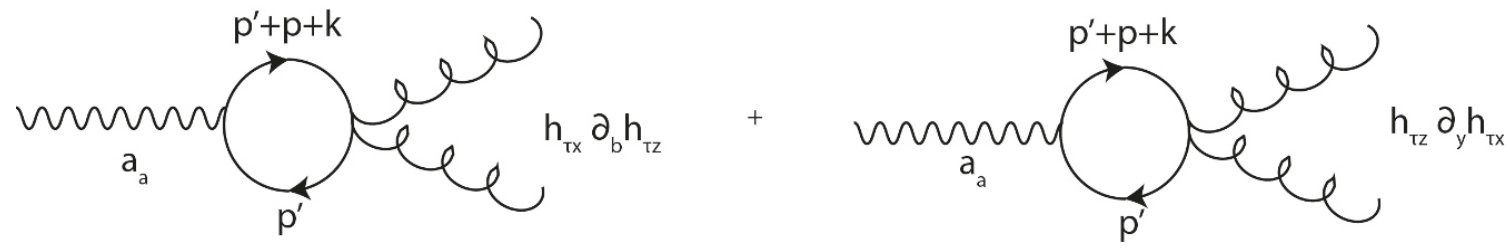

Figure 11. Contributions to $\zeta_{3}^{(6)}$ from contact terms.

Comparing the final result of the perturbative evaluation in (4.32) and the above equation we see that indeed the results for the coefficient $c_{\alpha}$ agrees with the gauge anomaly of a single Weyl fermion given in (4.18). Also we have the relation

$$
\tilde{c}_{m}^{6 d}=-8 \pi^{2} c_{\beta},
$$

which is again consistent with that obtained by the argument involving the consistency of the Euclidean vacuum.

Evaluation of $\boldsymbol{\lambda}_{\mathbf{3}}^{(\mathbf{6})}$. The last transport coefficient in $d=6$ is given by the following correlator

$$
\begin{aligned}
\left\langle T^{\mu \alpha} T^{\nu \beta} T^{\rho \sigma}\right\rangle=- & \frac{8}{\mathcal{Z}_{E}^{(0)}} \int \mathcal{D} \psi^{\dagger} \mathcal{D} \psi \exp \left(S_{E}^{(0)}\right) \times \\
& \times\left(\frac{\delta S_{E}}{\sqrt{g} \delta g_{\mu \alpha}} \frac{\delta S_{E}}{\sqrt{g} \delta g_{\nu \beta}} \frac{\delta S_{E}}{\sqrt{g} \delta g_{\rho \sigma}}+\frac{\delta^{2} S_{E}}{\sqrt{g} \delta g_{\mu \alpha} \sqrt{g} \delta g_{\nu \beta}} \frac{\delta S_{E}}{\sqrt{g} \delta_{\rho \sigma}}\right. \\
& +\frac{\delta^{2} S_{E}}{\sqrt{g} \delta g_{\mu \alpha} \sqrt{g} \delta g_{\rho \sigma}} \frac{\delta S_{E}}{\sqrt{g} \delta g_{\nu \beta}}+\frac{\delta S_{E}}{\sqrt{g} \delta g_{\mu \alpha}} \frac{\delta^{2} S_{E}}{\sqrt{g} \delta g_{\nu \beta} \sqrt{g} \delta_{\rho \sigma}} \\
& \left.+\frac{\delta^{3} S_{E}}{\sqrt{g} \delta g_{\mu \alpha} \sqrt{g} \delta g_{\nu \beta} \sqrt{g} \delta g_{\rho \sigma}}\right) .
\end{aligned}
$$

The coefficient in the Euclidean theory is given by

$$
\tilde{\lambda}_{3}^{(6)}=-\frac{3}{2} \lim _{p_{b}, k_{y} \rightarrow 0} \frac{-\left\langle T^{\tau a}(k+p) T^{\tau x}(-k) T^{\tau z}(-p)\right\rangle}{i p_{b} i k_{y}} .
$$

From the expansion of the action in (4.11), we see that only non-zero contributions to this correlator arise from

$$
\left\langle T^{\mu \alpha} T^{\nu \beta} T^{\rho \sigma}\right\rangle=A+B,
$$




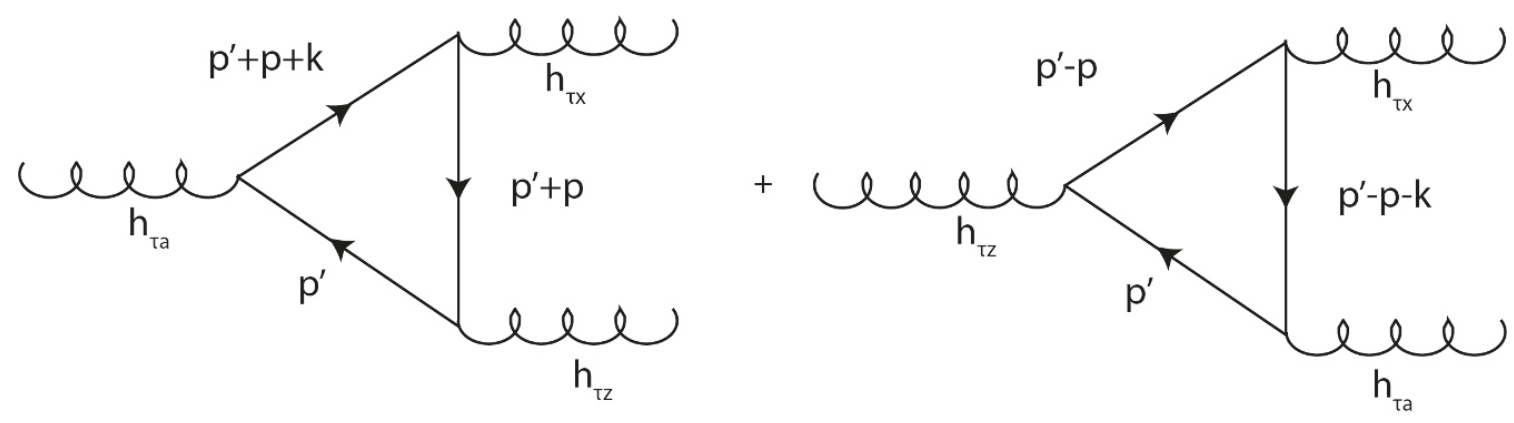

Figure 12. Contributions to $\lambda_{3}^{(6)}$ from Wick contractions of the stress tensor.

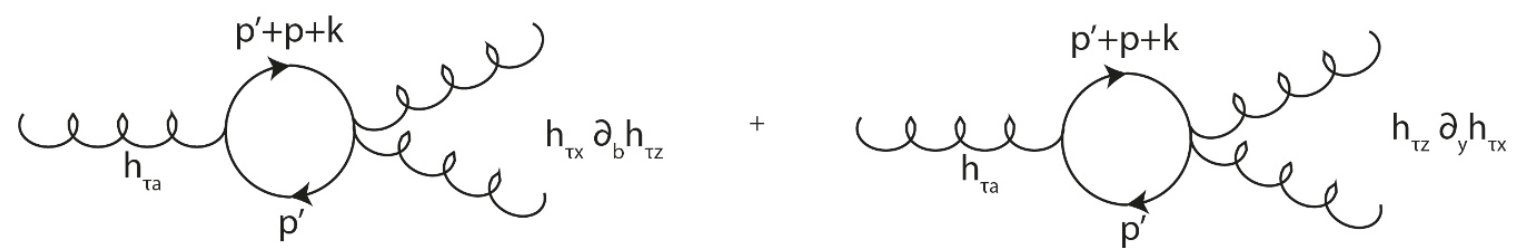

Figure 13. Contributions to $\lambda_{3}^{(6)}$ from contact terms.

$$
\begin{aligned}
A= & -\frac{8}{\mathcal{Z}_{E}^{(0)}} \int \mathcal{D} \psi^{\dagger} \mathcal{D} \psi \exp \left(S_{E}^{(0)}\right) \frac{\delta S_{E}}{\sqrt{g} \delta g_{\mu \alpha}} \frac{\delta S_{E}}{\sqrt{g} \delta g_{\nu \beta}} \frac{\delta S_{E}}{\sqrt{g} \delta g_{\rho \sigma}} \\
B= & -\frac{8}{\mathcal{Z}_{E}^{(0)}} \int \mathcal{D} \psi^{\dagger} \mathcal{D} \psi \exp \left(S_{E}^{(0)}\right)\left(\frac{\delta^{2} S_{E}}{\sqrt{g} \delta g_{\mu \alpha} \sqrt{g} \delta g_{\nu \beta}} \frac{\delta S_{E}}{\sqrt{g} \delta_{\rho \sigma}}\right. \\
& \left.+\frac{\delta^{2} S_{E}}{\sqrt{g} \delta g_{\mu \alpha} \sqrt{g} \delta g_{\rho \sigma}} \frac{\delta S_{E}}{\sqrt{g} \delta g_{\nu \beta}}+\frac{\delta S_{E}}{\sqrt{g} \delta g_{\mu \alpha}} \frac{\delta^{2} S_{E}}{\sqrt{g} \delta g_{\nu \beta} \sqrt{g} \delta_{\rho \sigma}}\right) .
\end{aligned}
$$

Term $A$ involves Wick contractions of the flat space stress tensor while $B$ is a contact term. From the explicit evaluation of term B in appendix B.4, it is seen that all terms in $B$ contribute equally.

The contributions to $A$ and one of the terms in $B$ are summarized in figure 12 and 13 respectively. The detailed evaluation of all the diagrams yields the following result

$$
\begin{aligned}
\tilde{\lambda}_{3}^{(6)} & =\frac{-3 i}{16 \pi^{3}} \int_{0}^{\infty} d p p_{1}^{3}\left(f\left(E_{p_{1}}-e \mu_{-}\right)-f\left(E_{p_{1}}+e \mu_{-}\right)\right) \\
& =\frac{-3 i}{64 \pi^{3}}\left(\frac{7 \pi^{4} T^{4}}{15}+2 e^{2} \pi^{2} T^{2} \mu_{-}^{2}+e^{4} \mu_{-}^{4}\right) .
\end{aligned}
$$

Writing down the transport coefficient in the Lorentzian theory using the relations in (4.5) gives

$$
\lambda_{3}^{(6)}=\frac{3}{64 \pi^{3}}\left(\frac{7 \pi^{4} T^{4}}{15}+2 e^{2} \pi^{2} T^{2} \mu_{-}^{2}+e^{4} \mu_{-}^{4}\right) .
$$

The importance of this transport coefficient is that it captures one of the pure gravitational anomaly $d=6$. Again following [12], we parametrize this coefficient as

$$
\lambda_{3}^{(6)}=9\left(-c_{\alpha} \mu_{-}^{4}+\tilde{c}_{m}^{6 d} T^{2} \mu_{-}^{2}+\tilde{c}_{g}^{6 d} T^{4}\right) .
$$


Comparing the result of the perturbative evaluation in (4.39), we see that the values of the gauge anomaly $c_{\alpha}$ and the coefficient $\tilde{c}_{m}^{6 d}$ is related to the mixed anomaly, as seen in the earlier correlators, while $\tilde{c}_{g}^{6 d}$ is given by

$$
\tilde{c}_{g}^{6 d}=\frac{7 \pi}{2880}
$$

Comparing with the coefficients of the pure gravitational anomaly of a Weyl fermion $c_{\gamma}$ given in (4.18), we obtain

$$
\tilde{c}_{g}^{6 d}=-\left(8 \pi^{2}\right)^{2} c_{\gamma} .
$$

This relation is what is expected from the replacement rule and the consistency of the Euclidean vacuum. ${ }^{14}$

\subsection{Chiral gravitinos}

Chiral gravitinos cannot be consistently coupled to a charge current. Therefore they contribute only to the pure gravitational anomaly and only to the transport coefficient $\lambda_{3}^{(6)}$. In this section, we show that analogous to the coefficient $\lambda^{(2)}$ for the chiral gravitino like theory $d=2$ in section 2.3 , the $\lambda_{3}^{(6)}$ for chiral gravitinos in $d=6$ do not obey the relation $\tilde{c}_{g}^{6 d}=-\left(8 \pi^{2}\right)^{2} c_{\gamma}$. We will identify the terms which do not contribute to the transport coefficient but contribute to the anomaly polynomial. The gauge fixed gravitino Lagrangian is given by

$$
\begin{aligned}
& S_{E}=S_{E}^{\mathrm{gr}}+S_{E}^{\mathrm{gh}}, \\
& S_{E}^{\mathrm{gr}}=\int d \tau d x^{5} \sqrt{g} e_{a}^{\mu} \psi_{\rho}^{\dagger} D_{\mu} P_{-} \psi^{\rho},
\end{aligned}
$$

where the covariant derivative is given by

$$
D_{\mu} \psi^{\rho}=\partial_{\mu} \psi^{\rho}+\frac{1}{2} \omega_{\mu c d} \sigma^{c d} \psi^{\rho}+\Gamma_{\mu \sigma}^{\rho} \psi^{\sigma} .
$$

The action $S_{E}^{\text {gh }}$ is the action of the corresponding ghosts. The contribution of all the ghosts can be accounted by subtracting the contribution of a chiral spinor. The action $S_{E}^{\mathrm{gr}}$ is similar to the action of 6 chiral fermions except that the covariant derivative in (4.44) contains the Christoffel symbol in addition to the spin connection. The linearization of the Christoffel symbol term results in the following interaction term in addition to the terms of the type present in (4.11).

$$
S_{\text {Christoffel }}^{(2)}=\frac{1}{2} \int d \tau d^{5} x \sqrt{g} e_{a}^{\mu} \psi_{\rho}^{\dagger} \gamma^{a} \eta^{\rho \alpha}\left(\partial_{\mu} h_{\alpha \sigma}+\partial_{\sigma} h_{\alpha \mu}-\partial_{\alpha} h_{\mu \sigma}+O\left(h^{2}\right)\right) \psi^{\sigma} .
$$

The linear terms in the metric modify the stress tensor. There are higher order terms in the expansion of the metric and one would have expected possible contributions to contact terms from them. However such terms do not have sufficient number of gamma matrices to contribute to the correlator involving 3 stress tensors. We need 6 gamma matrices, so

\footnotetext{
${ }^{14}$ We have not checked if the precise coefficient of $-\left(8 \pi^{2}\right)^{2}$ agrees with the normalizations of [12].
} 
that the correlator is non-zero. Any possible contact term from higher order terms in the above action have lesser number of gamma matrices. Therefore we conclude that the only difference of the chiral gravitino, compared to that of the chiral fermion, is that its flat space tensor acquires additional term, due to the linearization of the Christoffel symbol. The stress tensor is given by

$$
\begin{aligned}
T^{\mu \nu} & =T_{g 1}^{\mu \nu}+T_{g 2}^{\mu \nu}, \\
T_{g 1}^{\mu \nu} & =\frac{1}{2} \psi_{\rho}^{\dagger}(x)\left(\gamma^{\mu} \partial^{\nu}+\gamma^{\nu} \partial^{\mu}\right) P_{-} \psi^{\rho}(x) \\
T_{g 2}^{\mu \nu} & =-\frac{1}{2}\left(\partial _ { \sigma } \left(\psi^{\dagger, \sigma}(x) \gamma^{\mu} P_{-} \psi^{\nu}(x)-\partial_{\sigma}\left(\psi^{\mu \dagger}(x) \gamma^{\nu} P_{-} \psi^{\sigma}(x)+(\mu \leftrightarrow \nu)\right) .\right.\right.
\end{aligned}
$$

Note that the contribution $T_{\mu \nu}^{g 2}$ is due to the linearization of the Christoffel symbol. In momentum space the stress tensor is given by

$$
\begin{aligned}
T_{f l}^{\tau \mu}(k) & =T_{g 1}^{\tau s}(k)+T_{g 1}^{\tau s}(k), \\
T_{g 1}^{\tau s}(k) & =\frac{1}{2 \beta} \sum_{m} \int \frac{d^{5} p^{\prime}}{(2 \pi)^{5}}\left(\psi^{\dagger}\left(\omega_{m}, p^{\prime}-k\right)\left(\gamma^{\tau} i p_{s}^{\prime}+\gamma^{x} i \omega_{m}\right) P_{-} \psi^{\mu}\left(\omega_{m}, p^{\prime}\right),\right. \\
T_{g 2}^{\tau s}(k) & =i \frac{k_{\sigma}}{2 \beta} \sum_{m} \frac{d^{5} p^{\prime}}{(2 \pi)^{5}}\left(\psi^{\sigma \dagger}\left(\omega_{m}, p^{\prime}-p\right) \gamma^{\tau} P_{-} \psi^{s}\left(\omega_{m}, p^{\prime}\right)-\psi^{\tau^{\dagger}}\left(\omega_{m}, p^{\prime}-p\right) \gamma^{z} P_{-} \psi^{\sigma}\left(\omega_{m}, p^{\prime}\right)\right. \\
& +(\tau \leftrightarrow s)) .
\end{aligned}
$$

We can now evaluate the correlator

$$
\lambda_{3}^{(6)}=-\frac{3}{2} \lim _{p_{b}, k_{y} \rightarrow 0} \frac{-\left\langle T^{\tau a}(k+p) T^{\tau x}(-k) T^{\tau z}(-p)\right\rangle}{i p_{b} i k_{y}} .
$$

Apart from the contact terms arising from the linearization of the spin connection, we have to evaluate the following contribution to the correlator

$$
A=\left\langle T_{c l}^{\tau a}(k+p) T_{c l}^{\tau x}(-k) T_{c l}^{\tau z}(-p)\right\rangle .
$$

Substituting the expression for $T_{c l}^{\tau s}$ from (4.48), it can seen from the structure of the Wick contractions, that the only non-zero terms are

$$
\begin{aligned}
A= & A_{1}+A_{2} \\
A_{1}= & \left\langle T_{g 1}^{\tau a}(k+p) T_{g 1}^{\tau x}(-k) T_{g 1}^{\tau z}(-p)\right\rangle \\
A_{2}= & \left\langle T_{g 2}^{\tau a}(k+p) T_{g 2}^{\tau x}(-k) T_{g 1}^{\tau z}(-p)\right\rangle+\left\langle T_{g 2}^{\tau a}(k+p) T_{g 1}^{\tau x}(-k) T_{g 2}^{\tau z}(-p)\right\rangle \\
& +\left\langle T_{g 2}^{\tau a}(k+p) T_{g 2}^{\tau x}(-k) T_{g 2}^{\tau z}(-p)\right\rangle .
\end{aligned}
$$

After performing Wick contractions, the sum of the terms in $A_{2}$ organize themselves to

$$
\begin{aligned}
A_{2}= & -\frac{1}{4 \beta} \sum_{m} \int \frac{d^{5} p^{\prime}}{(2 \pi)^{5}} \times \\
& \times\left(p_{b}^{2} \operatorname{Tr}\left[\gamma^{a} S\left(\omega_{m}, p^{\prime}+p+k\right)\left(\gamma^{\tau} p_{x}^{\prime}+\gamma^{x} i \omega_{m}\right) S\left(\omega_{m}, p^{\prime}+p\right) \gamma^{z} S\left(\omega_{m}, p^{\prime}\right) P_{+}\right]\right. \\
& +p_{b}^{2} \operatorname{Tr}\left[\gamma^{z} S\left(\omega_{m}, p^{\prime}-p\right)\left(\gamma^{\tau} p_{x}^{\prime}+\gamma^{x} i \omega_{m}\right) S\left(\omega_{m}, p^{\prime}-p-k\right) \gamma^{a} S\left(\omega_{m}, p^{\prime}\right) P_{+}\right]
\end{aligned}
$$




$$
\begin{aligned}
& +k_{y}^{2} \operatorname{Tr}\left[\gamma^{a} S\left(\omega_{m}, p^{\prime}+p+k\right) \gamma^{x} S\left(\omega_{m}, p^{\prime}+p\right)\left(\gamma^{\tau} p_{z}^{\prime}+\gamma^{z} i \omega_{m}\right) S\left(\omega_{m}, p^{\prime}\right) P_{+}\right] \\
& \left.+k_{y}^{2} \operatorname{Tr}\left[\left(\gamma^{\tau} p_{z}^{\prime}+\gamma^{z} i \omega_{m}\right) S\left(\omega_{m}, p^{\prime}-p\right) \gamma^{x} S\left(\omega_{m}, p^{\prime}-p-k\right) \gamma^{a} S\left(\omega_{m}, p^{\prime}\right) P_{+}\right]\right)
\end{aligned}
$$

After inserting the propagator and performing the Matsubara sums, it can be seen that each of the terms in $A_{2}$ is proportional to $p_{b} k_{y}$. Therefore, the leading contribution to the transport coefficient from $A_{2}$ is of the form

$$
\lim _{p_{b}, k_{y} \rightarrow 0} \frac{A_{2}}{i p_{b} i k_{y}}=O\left(p_{b}^{2}\right)+O\left(k_{y}^{2}\right) .
$$

The entire contribution from $A_{2}$ to the transport coefficient vanishes in the zero momentum limit. This is the same mechanism by which, the contribution from terms arising from the linearization of the Christoffel symbol to $\lambda^{(2)}$ in the gravitino like theory in $d=2$, vanishes. The contribution from $A_{1}$ is 6 times that of a chiral fermion. The contribution from the contact terms arising from the linearization of the spin connection is also 6 times that of a chiral fermion. To these contributions we must subtract the contribution of a single chiral fermion to account for the ghosts. Using the results of the the section 4.1 equation (4.39), we conclude that the contribution of a chiral gravitino to $\lambda_{3}^{(6)}$ is given by

$$
\lambda_{3, \text { gravitinos }}^{(6)}=5 \times \frac{3}{64 \pi^{3}} \frac{7 \pi^{4} T^{4}}{15}=9 \tilde{c}_{g, \text { gravitinos }}^{6 d} .
$$

Here the last equality arises from the parametrization following from the replacement rule.

Now let us compare the transport coefficient to the coefficient of the pure gravitational anomaly. The terms arising from the linearization of the Christoffel symbol do give a contribution to the anomaly polynomial of the gravitino [23]. ${ }^{15}$ In fact such terms give rise to the interaction of the magnetic moment of the gravitino with the gravitational field. These terms modify the gravitational anomaly coefficient $c_{\gamma}$ for gravitinos which is given by

$$
c_{\gamma, \text { gravitinos }}=-275 \times \frac{1}{184320 \pi^{3}} .
$$

Comparing (4.54) and (4.55), we see that gravitinos do not obey the relation $\tilde{c}_{g}^{6 d}=-\left(8 \pi^{2}\right)^{2} c_{\gamma}$, which is argued from the consistency of the Euclidean vacuum in [12]. Here we have identified the terms which contribute to the anomaly polynomial but not to the transport coefficient. From our analysis in $d=2, d=6$ we see that gravitinos contribute to correlators involving only the stress tensor, since they are not charged. Their contribution is essentially $d-1$ times that of a chiral fermion to the transport coefficient. This is the same conclusion arrived at, by analysis of the helicity of the thermal state, in [19].

\section{Hydrodynamic modes in $d=2$ Weyl gas}

A charged $1+1$ dimensional relativistic fluid admits 2 hydrodynamic modes, the sound mode and the charge diffusion mode. The leading dispersion relation for these modes, without anomalous terms in the constitutive relations for the stress tensor and the charge

\footnotetext{
${ }^{15}$ See equation (35) of [23].
} 


\begin{tabular}{|c|c|c|c|}
\hline Dimension & Correlator & Species & Value \\
\hline $\mathrm{d}=6$ & $\zeta_{1}^{(6)}$ & Fermions & $\frac{3 e^{4}}{64 \pi^{3}} \mu_{-}$ \\
\hline & $\zeta_{2}^{(6)}$ & Fermions & $\frac{3 e^{2}}{32 \pi^{3}}\left(e^{2}\left(\mu_{-}\right)^{2}+\frac{\pi^{2} T^{2}}{3}\right)$ \\
\hline & $\zeta_{3}^{(6)}$ & Fermions & $\frac{e}{16 \pi^{3}}\left[\left(e \mu_{-}\right)^{3}+e \pi^{2} T^{2} \mu_{-}\right]$ \\
\hline & $\lambda_{3}^{(6)}$ & Fermions & $\frac{3}{64 \pi^{3}}\left(\frac{7 \pi^{4} T^{4}}{15}+2 e^{2} \pi^{2} T^{2}\left(\mu_{-}\right)^{2}+e^{4} \mu_{-}^{4}\right)$ \\
\hline & $\lambda_{3}^{(6)}$ & Gravitinos & $\frac{15}{64 \pi^{3}}\left(\frac{7 \pi^{4} T^{4}}{15}\right)$ \\
\hline
\end{tabular}

Table 2. Transport coefficients in $d=6$.

current, was obtained in [24]. In this section we would like to show that due to the presence of anomalous terms, proportional to $\zeta^{(2)}$ and $\lambda^{(2)}$ in the constitutive relation, the charge diffusion mode acquires a non-zero speed. We then apply the discussion to the case of ideal $d=2$ Weyl gas, held at a chiral chemical potential $\mu_{-}$. As seen in section 2 , such a gas has a non-zero value for $\zeta^{(2)}$ and $\lambda^{(2)}$. The speed of sound for this gas remains the same as the speed of light, however the speed of the charge diffusion mode is non-zero and is equal half the speed of light.

Consider a relativistic fluid in $d=2$ with anomalous transport $\zeta^{(2)}$ and $\lambda^{(2)}$ non-zero. The constitutive relations to the first derivative in velocity in the Lorentz frame is given by

$$
\begin{aligned}
T^{\mu \nu} & =(\epsilon+P) u^{\mu} u^{\nu}-P \eta^{\mu \nu}-\xi\left(u^{\mu} u^{\nu}-\eta^{\mu \nu}\right) \partial_{\lambda} u^{\lambda}, \\
j^{\mu} & =\rho u^{\mu}-\sigma T\left(\eta^{\mu \nu}-u^{\mu} u^{\nu}\right) \partial_{\nu}\left(\frac{\mu}{T}\right)+\zeta_{v} \epsilon^{\mu \nu} u_{\nu} .
\end{aligned}
$$

The signature of the metric is given by $\eta_{\mu \nu}=\operatorname{diag}(1,-1) . u^{\mu}$ is the velocity field with $u^{\mu} u_{\mu}=1, \xi$ is the bulk viscosity, $\sigma$ the conductivity and $\zeta_{v}$ is the anomalous transport coefficient. $\epsilon, P, \rho, \mu$ refer to the energy density, pressure, charge density and chemical potential respectively. $\zeta_{v}$ in the Lorentz frame is related to the transport coefficients in the anomaly frame by

$$
\zeta_{v}=\zeta^{(2)}-\frac{\rho}{\epsilon+P} \lambda^{(2)}
$$

To study linearized hydrodynamic modes, consider small fluctuations from the rest frame with velocity field $\left(1, v^{z}\right)\left(\left|v_{z}\right| \ll 1\right)$. We also have fluctuations in the energy density, pressure which are related to fluctuations in the stress tensor and charge current, using the constitutive relations in (5.1). These are given by

$$
\begin{aligned}
& \delta T^{00}=\delta \epsilon, \\
& \delta T^{0 z}=(\epsilon+P) \delta u^{z}, \\
& \delta T^{z z}=\delta P-\frac{\xi \partial_{z} \delta T^{0 z}}{\epsilon+P}, \\
& \delta j^{0}=\delta \rho-\zeta_{v} \delta u^{z}, \\
& \delta j^{z}=\rho \delta u^{z}-\sigma T \partial_{z}(\delta \bar{\mu})-\delta \zeta_{v} .
\end{aligned}
$$

It is convenient to use the energy density $\epsilon$ and charge density $\rho$ as independent variables. We can write the variation in chemical potential and pressure as

$$
\delta \bar{\mu}=\partial_{\epsilon} \bar{\mu} \delta T^{00}+\partial_{\rho} \bar{\mu} \delta \rho, \quad \delta P=\partial_{\epsilon} P \delta T^{00}+\partial_{\rho} P \delta \rho .
$$


where $\bar{\mu}=\mu / T$. The hydrodynamic modes are eigen modes of the linearized equations of hydrodynamics, which are given by

$$
\partial_{\mu} \delta T^{\mu \nu}=0, \quad \partial_{\mu} \delta j^{\mu}=0 .
$$

Here we set the background gauge field strength, including its fluctuations, to zero. Writing out these equations in Fourier space, ${ }^{16}$ eliminating $\delta T^{z z}$, and $\delta u^{z}$ using (5.3), we obtain the following set of equations from the conservation equation for the stress tensor

$$
\begin{aligned}
-i \omega \delta T^{00}+i k \delta T^{0 z} & =0, \\
-i \omega \delta T^{0 z}+i k\left(\partial_{\epsilon} P \delta T^{00}+\partial_{\rho} P \delta \rho\right)+k^{2} \xi \frac{\delta T^{0 z}}{\epsilon+P} & =0 .
\end{aligned}
$$

Now using the first equation in (5.6), to eliminate $\delta T^{0 z}$, we obtain

$$
\delta \rho\left(\partial_{\rho} P i k\right)+\delta T^{00}\left(\frac{-i \omega^{2}}{k}+\frac{\omega k \xi}{\epsilon+P}+i k \partial_{\epsilon} P\right)=0 .
$$

A similar analysis of the conservation equation for the current yields

$$
\begin{aligned}
\delta \rho(-i \omega+ & \left.k^{2} \sigma T \partial_{\rho} \bar{\mu}-i k \partial_{\rho} \zeta_{v}\right) \\
& +\delta T^{00}\left(k^{2} \sigma T \partial_{\epsilon} \bar{\mu}+\frac{i \omega \rho}{\epsilon+P}-i k \partial_{\epsilon} \zeta_{v}+\frac{i \omega^{2} \zeta_{v}}{k(\epsilon+P)}\right)=0 .
\end{aligned}
$$

From the homogeneous equations (5.7) and (5.8), we see that to obtain a non-trivial solution for the fluctuations $\delta \rho, \delta T^{00}$ we must have the condition

$$
\begin{aligned}
& \left(\partial_{\rho} P i k\right)\left(k^{2} \sigma T \partial_{\epsilon} \bar{\mu}+\frac{i \omega \rho}{\epsilon+P}-i k \partial_{\epsilon} \zeta_{v}+\frac{i \omega^{2} \zeta_{v}}{k(\epsilon+P)}\right) \\
& \quad-\left(\frac{-i \omega^{2}}{k}+\frac{\omega k \xi}{\epsilon+P}+i k \partial_{\epsilon} P\right)\left(-i \omega+k^{2} \sigma T \partial_{\rho} \bar{\mu}-i k \partial_{\rho} \zeta_{v}\right)=0 .
\end{aligned}
$$

To obtain solutions which satisfy the condition in (5.9), we assume the dispersion relation of the form

$$
\omega=v k-i D k^{2}+O\left(k^{3}\right) .
$$

Substituting this anstaz into (5.9), we see that the velocity $v$ is determined by the following cubic equation

$$
v^{3}+v^{2}\left(-\frac{\partial_{\rho} P \zeta_{v}}{\epsilon+P}+\partial_{\rho} \zeta_{v}\right)-v\left(\frac{\rho \partial_{\rho} P}{\epsilon+P}+\partial_{\epsilon} P\right)-\partial_{\epsilon} P \partial_{\rho} \zeta_{v}+\partial_{\rho} P \partial_{\epsilon} \zeta_{v}=0
$$

This equation is obtained by setting the coefficient of the $O\left(k^{2}\right)$ term to zero on substituting the anstaz (5.10) in (5.9). As a simple check of this equation, consider setting the anomalous transport coefficients $\zeta_{v}=0$, then the equation reduces to

$$
v^{2}-\left(\frac{\rho \partial_{\rho} P}{\epsilon+P}+\partial_{\epsilon} P\right) v=0 .
$$

\footnotetext{
${ }^{16}$ For eg. Fourier mode of $\delta T^{z z}=e^{-i \omega t+i k z} \delta T^{z z}(\omega, k)$.
} 
The three solutions to this equation are

$$
v= \pm\left(\frac{\rho \partial_{\rho} P}{\epsilon+P}+\partial_{\epsilon} P\right)^{\frac{1}{2}}, \quad v=0 .
$$

The first two roots correspond to the speed of sound and the last one is the speed of the charge diffusion mode. Now one can show for $\zeta_{v} \neq 0$ but small, there are corrections to both speed of sound and the speed of the charge diffusion mode that depend on $\zeta_{v}$. On further analysis, one can show that the the dissipative constant $D$ for both the sound and the charge diffusion mode receives corrections on turning on $\zeta_{v}$.

We will now show for the ideal Weyl gas the three roots of (5.11) are $v= \pm 1, v=-1 / 2$. The thermodynamics of the ideal Weyl gas is given by [18]

$$
\begin{aligned}
& \epsilon=\frac{e^{2} \mu_{-}^{2}}{4 \pi}+\frac{T^{2} \pi}{12}=P, \\
& \rho=\frac{e^{2} \mu_{-}}{2 \pi} .
\end{aligned}
$$

Using (5.2) and the values of $\zeta^{(2)}$ and $\lambda^{(2)}$ we obtain the transport coefficient to be given by

$$
\begin{aligned}
\zeta_{v} & =\frac{e^{2} \mu_{-}}{2 \pi}-\frac{1}{4 \pi} \frac{\rho}{P+\epsilon}\left(e^{2} \mu_{-}^{2}+\frac{\pi^{2} T^{2}}{3}\right), \\
& =\frac{\rho}{2} .
\end{aligned}
$$

To obtain the last equality we have used the expression for energy density and $\epsilon=P$. Since the ideal Weyl gas is conformal and there are no interactions the bulk viscosity $\xi$ as well as the conductivity $\sigma$ vanishes. Now from the relations in (5.14) and (5.15) we see that

$$
\partial_{\rho} P=0, \quad \partial_{\epsilon} P=1, \quad \partial_{\rho} \zeta_{v}=\frac{1}{2}, \quad \partial_{\epsilon} \zeta_{v}=0 .
$$

Substituting these values in equation (5.11) for the velocity of the modes we obtain

$$
v^{3}+\frac{1}{2} v^{2}-v-\frac{1}{2}=0 .
$$

Therefore the 3 roots are

$$
v= \pm 1,-\frac{1}{2}
$$

As we discussed earlier, the first two roots correspond to the sound mode which moves with the velocity of light. The last root corresponds to the speed of the charge diffusion mode. Note that there is a unique sign for this speed, this is because of the definite chirality of the Weyl fermions. If the we had considered Weyl fermions with opposite chirality the sign would have been positive.

It will be interesting to explore this phenomenon further to see if it is observable in realistic low dimensional quantum systems like quantum wires. It will be also interesting to study the effect of the anomalous transport coefficients for other relativistic systems in $1+1$ dimensions. One such example is the charged D1-brane which was studied in [24]. 


\section{Conclusions}

We have evaluated all the leading parity odd transport coefficients for chiral fermions in $d=2,4,6$ as well as chiral bosons in $d=2$ at one loop. The analysis was done using finite temperature field theory. We have seen that chiral fermions as well as chiral bosons in $d=2$ obey the replacement rule of [12] which relates the coefficient of anomalies including both mixed and pure gravitational anomalies to the respective transport coefficient. As far as we are aware the calculations in $d=2$ is the first direct perturbative evaluation of transport coefficients using the Kubo formula. The calculations in $d=4,6$ serve as an alternative approach as well as a check of the calculations done in [22] which involved SchwingerKeldysh propagators. We have also kept track of the contributions from the gravitational anomalies which was ignored in [22]. For a chiral gravitino like theory in $d=2$ as well as chiral gravitinos in $d=6$ the relationship between the pure gravitational anomaly and the corresponding transport coefficient found using the argument involving the consistency of the Euclidean vacuum breaks down. We have identified the mechanism of how this occurs in the perturbative analysis. We also developed a simple method to perform the angular integrals that occur in the transport coefficients which involves taking the zero momentum limit first and the use of an $i \epsilon$ prescription. Finally we have shown that the charge diffusion mode of the ideal Weyl gas in $d=2$ at finite chemical potential acquires a speed which is $1 / 2$ the speed of light due to the presence of anomalous transport coefficients.

There are several directions in which the work done in this paper can be extended. It will be interesting to extend the analysis done in this paper to all even dimensions. A more challenging task would be to evaluate the contribution of self dual tensors in $d=4 k+2$ for $k>0$ to the transport coefficient Though the coupling of these tensors to the charge current is not explicitly known, one can evaluate contributions related to the pure gravitational anomaly of these tensors. Direct holographic checks of these transport coefficients in $A d S_{3}, A d S_{7}$ backgrounds similar to the ones done in $A d S_{5}$ in [9] is another direction direction of interest. Finally it will be interesting to study to what extent these parity odd transport coefficients are one loop exact analogous to what was done for the coefficient $\zeta_{2}^{(4)}$ in $d=4$ by [11].

\section{Acknowledgments}

We thank Sayantani Bhattacharyya, Zohar Komargodski, R. Loganayagam, Diptiman Sen, Aninda Sinha and Amos Yarom for discussions on various issues related to this work. S.D.C. thanks Edward Witten for the discussion during the poster session of Strings 2015 which helped us to clarify our understanding of the gravitino like theory in $d=2$. We also thank Kristan Jensen for useful correspondence.

\section{A Moments of statistical distributions}

To evaluate the various transport coefficients of interest in this paper we require several moments of the Fermi-Dirac distribution. Let $f(p)$ be the Fermi-Dirac distribution which 
is given by

$$
f(p)=\frac{1}{e^{\beta p}+1}
$$

The results for the following moments are used at several instances in the paper

$$
\begin{aligned}
\int_{0}^{\infty} d p[f(p-e \mu)-f(p+e \mu)] & =e \mu \\
\int_{0}^{\infty} d p p[f(p-e \mu)+f(p+e \mu)] & =\frac{1}{2}\left(e^{2} \mu^{2}+\frac{\pi^{2} T^{2}}{3}\right) \\
\int_{0}^{\infty} d p p^{2}[f(p-e \mu)-f(p+e \mu)] & =\frac{1}{3}\left(e^{3} \mu^{3}+e \pi^{2} T^{2} \mu\right) \\
\int_{0}^{\infty} d p p^{3}[f(p-e \mu)+f(p+e \mu)] & =\frac{1}{4}\left(\frac{7 \pi^{4} T^{4}}{15}+2 e^{2} \pi^{2} T^{2} \mu^{2}+e^{4} \mu^{4}\right)
\end{aligned}
$$

These integrals are derived in [18]. Let $b(p)$ by the Bose-Einstein distribution which is given by

$$
b(p)=\frac{1}{e^{\beta p}-1}
$$

Then the following integral is required in the analysis of chiral bosons.

$$
\int_{0}^{\infty} d p p b(p)=\frac{\pi^{2} T^{2}}{6}
$$

\section{B Evaluation of transport coefficients in $d=6$}

In this appendix we detail the calculations involved in obtaining the leading parity odd transport coefficients in $d=6$.

\section{B.1 Contributions to $\zeta_{1}^{(6)}$}

Before we begin, let us recall the kinematic set up of the correlator considered in (4.13). This kinematic configuration will be the same for all the other correlators considered in $d=6$. There are two external momenta $p, k$. We set the frequency components of both these external momenta to zero. The momentum vector $p$ has a non-zero component only along the $b$ direction while momentum vector $k$ has component only along the $y$ direction. While performing the integration over the spatial directions of the internal momentum labelled as $p^{\prime}$ it is convenient to parametrize its components in terms of angular variables as

$$
\begin{array}{ll}
p_{b}^{\prime}=\left|p^{\prime}\right| \cos \phi_{1}, \quad p_{y}^{\prime}=\left|p^{\prime}\right| \sin \phi_{1} \cos \phi_{2}, & p_{x}^{\prime}=\left|p^{\prime}\right| \sin \phi_{1} \sin \phi_{2} \cos \phi_{3}, \\
p_{z}^{\prime}=\left|p^{\prime}\right| \sin \phi_{1} \sin \phi_{2} \sin \phi_{3} \cos \phi_{4}, & p_{a}^{\prime}=\left|p^{\prime}\right| \sin \phi_{1} \sin \phi_{2} \sin \phi_{3} \sin \phi_{4} .
\end{array}
$$

We choose orientation of the axis so that

$$
\operatorname{Tr}\left(\gamma^{p} \gamma^{q} \gamma^{r} \gamma^{s} \gamma^{t} \gamma^{u} \gamma_{c}\right)=-8 \epsilon^{p q r s t u}, \quad \epsilon^{\tau a z x y b}=i .
$$

With this kinematic configurations, let us write down the expression for the various energies which will occur in the expression for the propagators.

$$
E_{p^{\prime}+p}=\left(\left|p^{\prime}\right|^{2}+|p|^{2}+2|p|\left|p^{\prime}\right| \cos \phi_{1}\right)^{\frac{1}{2}},
$$




$$
\begin{aligned}
E_{p^{\prime}+k} & =\left(\left|p^{\prime}\right|^{2}+|k|^{2}+2|k|\left|p^{\prime}\right| \sin \phi_{1} \cos \phi_{2}\right)^{\frac{1}{2}}, \\
E_{p^{\prime}+p+k} & =\left(\left|p^{\prime}\right|^{2}+|k|^{2}+|p|^{2}+2|k|\left|p^{\prime}\right| \sin \phi_{1} \cos \phi_{2}+2|p|\left|p^{\prime}\right| \cos \phi_{1}\right)^{\frac{1}{2}} .
\end{aligned}
$$

For convenience in notation we will also define

$$
i \omega_{-}=i \omega_{m}+e\left(\mu-\mu_{c}\right), \quad \omega_{m}=(2 m+1) \pi T .
$$

Now substituting the expression for the propagators from (3.21), into (4.15), we obtain

$$
\begin{aligned}
& \left\langle j^{a}(p+k) j^{x}(-k) j^{z}(-p)\right\rangle=\frac{e^{3}}{2^{3} \beta} \sum_{m} \int \frac{d^{5} p^{\prime}}{(2 \pi)^{5}} \times \\
& \times\left(\sum_{t, v, u= \pm} \operatorname{Tr}\left[\gamma^{a} \gamma^{\alpha} \gamma^{x} \gamma^{\beta} \gamma^{z} \gamma^{\nu} P_{+}\right] \Delta_{t}\left(i \omega_{-}, p^{\prime}+k+p\right)\left(\hat{p^{\prime}}+\hat{k}+\hat{p}\right)_{(t, \alpha)} \times\right. \\
& \left.\quad \times \Delta_{u}\left(i \omega_{-}, p^{\prime}+p\right)\left(\hat{p}^{\prime}+\hat{p}\right)_{(\beta, u)} \Delta_{v}\left(i \omega_{-}, p^{\prime}\right) \hat{p}_{(\nu, v)}^{\prime}\right)+ \\
& +(\text { Exchange term }) .
\end{aligned}
$$

The exchange term is the second term in equation (4.15), arising due to the second Wick contraction. We can obtain the exchange term from the term written down by the performing the following operation on the first term written in (B.5).

$$
\begin{aligned}
a & \rightarrow z, & z & \rightarrow a, \\
\left(p^{\prime}+p+k\right) & \rightarrow\left(p^{\prime}-p\right), & \left(p^{\prime}+p\right) & \rightarrow\left(p^{\prime}-p-k\right) .
\end{aligned}
$$

Performing the trace over the $\gamma$ matrices, we obtain

$$
\begin{aligned}
\operatorname{Tr}\left[\gamma^{a} \gamma^{\alpha} \gamma^{x} \gamma^{\beta} \gamma^{z} \gamma^{\nu} P_{+}\right]\left(\hat{p}^{\prime}+\hat{k}+\hat{p}\right)_{(t, \alpha)}\left(\hat{p}^{\prime}+\hat{p}\right)_{(u, \beta)}\left(\hat{p}^{\prime}\right)_{(v, \nu)} \\
=\left(\frac{4 t u v p_{a}^{\prime}\left(p^{\prime}+p\right)_{z}\left(p^{\prime}-q\right)_{x}}{E_{p^{\prime}} E_{\left(p^{\prime}+p\right)} E_{\left(p^{\prime}-q\right)}}+\text { permutations in }(a, z, x)\right) \\
\quad+\left(\frac{4 t u\left(k_{y} p_{b}^{\prime}+p_{b} k_{y}\right)}{E_{p^{\prime}+p+k} E_{p^{\prime}+p}}-\frac{4 u v p_{y}^{\prime} p_{b}}{E_{p^{\prime}} E_{p^{\prime}+p}}-\frac{4 t v\left(k_{y} p_{b}^{\prime}-p_{b} p_{y}^{\prime}\right)}{E_{p^{\prime}+p+k} E_{p^{\prime}}}\right) .
\end{aligned}
$$

The next step is to perform the following four integrals

$$
\begin{aligned}
\frac{-A_{1}}{i p_{b} i k_{y}}=\frac{e^{3}}{p_{b} k_{y} 2^{3} \beta} \sum_{m} \int \frac{d^{5} p^{\prime}}{(2 \pi)^{5}} \sum_{t, v, u= \pm}\left(\frac{4 p_{a}^{\prime}\left(p^{\prime}+p\right)_{z}\left(p^{\prime}+p+k\right)_{x}}{E_{p^{\prime}} E_{\left(p^{\prime}+p\right)} E_{\left(p^{\prime}-q\right)}}+\text { permutations }\right) \times \\
\frac{-A_{2}}{i p_{b} i k_{y}}=\frac{e^{3}\left(i \omega_{-}, p^{\prime}+k+p\right) \Delta_{u}\left(i \omega_{-}, p^{\prime}+p\right) \Delta_{v}\left(i \omega_{-}, p^{\prime}\right),}{p_{b} k_{y} 2^{3} \beta} \sum_{m} \int \frac{d^{5} p^{\prime}}{(2 \pi)^{5}} \sum_{t, v, u= \pm} \frac{4 t u p_{b} k_{y}}{E_{p^{\prime}+p+k} E_{p^{\prime}+p}} \times \\
\begin{aligned}
\quad \times \Delta_{t}\left(i \omega_{-}, p^{\prime}+k+p\right) \Delta_{u}\left(i \omega_{-}, p^{\prime}+p\right) \Delta_{v}\left(i \omega_{-}, p^{\prime}\right), \\
\frac{-A_{3}}{i p_{b} i k_{y}}=\frac{e^{3}}{p_{b} k_{y} 2^{3} \beta} \sum_{m} \int \frac{d^{5} p^{\prime}}{(2 \pi)^{5}} \sum_{t, v, u= \pm}\left(\frac{4 t u k_{y} p_{b}^{\prime}}{E_{p^{\prime}+p+k} E_{p^{\prime}+p}}-\frac{4 t v k_{y} p_{b}^{\prime}}{E_{p^{\prime}+p+k} E_{p^{\prime}}}\right) \times \\
\times \Delta_{t}\left(i \omega_{-}, p^{\prime}+k+p\right) \Delta_{u}\left(i \omega_{-}, p^{\prime}+p\right) \Delta_{v}\left(i \omega_{-}, p^{\prime}\right),
\end{aligned}
\end{aligned}
$$




$$
\begin{aligned}
\frac{-A_{4}}{i p_{b} i k_{y}}=\frac{e^{3}}{p_{b} k_{y} 2^{3} \beta} \sum_{m} \int \frac{d^{5} p^{\prime}}{(2 \pi)^{5}} \sum_{t, v, u= \pm}\left(-\frac{4 u v p_{y}^{\prime} p_{b}}{E_{p^{\prime}} E_{p^{\prime}+p}}+\frac{4 t v p_{b} p_{y}^{\prime}}{E_{p^{\prime}+p+k} E_{p^{\prime}}}\right) \\
\left.\times \Delta_{t}\left(i \omega_{-}, p^{\prime}+k+p\right)\right] \Delta_{u}\left(i \omega_{-}, p^{\prime}+p\right) \Delta_{v}\left(i \omega_{-}, p^{\prime}\right) .
\end{aligned}
$$

Here we have divided by the external momenta and multiplied by a negative sign in each term so that we obtain terms directly related to the transport coefficient of interest. Each of the terms involve a sum over Fermionic Matsubara frequencies. They are evaluated using the following expression

$$
\begin{aligned}
& \frac{1}{\beta} \sum_{m} \Delta_{t}\left(i \omega_{-}, p^{\prime}+p+k\right) \Delta_{u}\left(i \omega_{-}, p^{\prime}+p\right) \Delta_{v}\left(i \omega_{-}, p^{\prime}\right) \\
& =\frac{t f\left(E_{p^{\prime}+p+k}-t e \mu_{-}\right)}{\left(t E_{p^{\prime}+p+k}-v E_{p^{\prime}}\right)\left(t E_{p^{\prime}+p+k}-u E_{p^{\prime}+p}\right)}+\frac{u f\left(E_{p^{\prime}+p}-u e \mu_{-}\right)}{\left(u E_{p^{\prime}+p}-t E_{p^{\prime}+p+k}\right)\left(u E_{p^{\prime}+p}-v E_{p^{\prime}}\right)} \\
& \quad \quad+\frac{v f\left(E_{p^{\prime}}-v e \mu_{-}\right)}{\left(v E_{p^{\prime}}-t E_{p^{\prime}+p+k}\right)\left(v E_{p^{\prime}}-u E_{p^{\prime}+p}\right)} \\
& \equiv \mathcal{M} . \quad
\end{aligned}
$$

In arriving at the above sum, we have neglected terms independent of the temperature and chemical potential. An important observation from (B.3), is that all terms in the Matsubara sum is independent of the angles $\phi_{4}$ due to our choice of directions of the external momenta. Let us first evaluate $A_{1}$. The numerator is proportional to $p_{a}^{\prime} p_{x}^{\prime} p_{z}^{\prime}$. After substituting for these components from (B.1), we that the numerator is proportional to $\sin \phi_{4} \cos \phi_{4}$. Then on performing the angular integral over $\phi_{4}$, this term vanishes since each term in the Matsubara sum in (B.9) is independent of $\phi_{4}$.

The term $A_{2}$ term is given by

$$
\frac{-L_{2}}{i p_{b} i k_{y}}=\frac{e^{3}}{p_{b} k_{y} 2^{3} \beta} \sum_{\omega_{m}} \int \frac{d^{5} p_{1}}{(2 \pi)^{5}} \sum_{t, v, u= \pm} \frac{4 t u\left[p_{b} k_{y}\right]}{E_{p_{1}+p+k} E_{p_{1}+p}} \times \mathcal{M} .
$$

We perform the sum over a pair from $\{t, u, v\}$ in each of the 3 terms in the Matsubara sum $\mathcal{M}$ as follows. For the first term in the $\mathcal{M}$ we sum over the pair $u, v$, for the 2nd term the pair $t, v$ and for the 3 rd term we sum over the pair $t, u$. We can then change variables of the $p^{\prime}$ integration so that the numerator in each of the terms in the Matsubara sum is a function of the integration variable $p^{\prime}$. For instance in the first term we substitute $p^{\prime}+p+k=\tilde{p}^{\prime}$, while in the second term we substitute $p^{\prime}+p=\tilde{p}^{\prime}$. This ensures that the numerator of each term in $\mathcal{M}$ is a function of $E_{p^{\prime}}$ with $p^{\prime}$ being the integration variable. After performing these manipulations we obtain

$$
\begin{aligned}
\frac{-A_{2}}{i p_{b} i k_{y}}= & \frac{2 e^{3}}{(2 \pi)^{5}} \sum_{t= \pm} \int d^{5} p^{\prime} t f\left(E_{p^{\prime}}-t e \mu_{-}\right) \\
& \frac{1}{\left(E_{p^{\prime}}^{2}-E_{p^{\prime}+p}^{2}\right)\left(E_{p^{\prime}}^{2}-E_{p^{\prime}+k+p}^{2}\right)}+\frac{1}{\left(E_{p^{\prime}}^{2}-E_{p^{\prime}-k}^{2}\right)\left(E_{p^{\prime}}^{2}-E_{p^{\prime}+p}^{2}\right)} \\
& \frac{1}{\left(E_{p^{\prime}}^{2}-E_{p^{\prime}+k}^{2}\right)\left(E_{p^{\prime}}^{2}-E_{p^{\prime}+p+k}^{2}\right)} .
\end{aligned}
$$


We can now take the $p, k \rightarrow 0$ limit and expand each term in the above expression as a series in $p, k$. The order of limit in these expansions does not matter. The leading singular term and finite terms are

$$
\begin{aligned}
\lim _{p_{b}, k_{y} \rightarrow 0} \frac{-A_{2}}{i p_{b} i k_{y}}= & \frac{2 e^{3}}{(2 \pi)^{5}} \sum_{t= \pm} \int d^{5} p^{\prime} t f\left(E_{p^{\prime}}-t e \mu_{-}\right) \\
& \times\left(\frac{1}{8 p^{\prime 4} \cos ^{2} \phi_{1} \sin ^{2} \phi_{1} \cos ^{2} \phi_{2}}-\frac{1}{4 p_{b} p_{1}^{3} \cos ^{2} \phi_{1} \sin \phi_{1} \cos \phi_{2}}\right) \\
& +O\left(p_{b}, k_{y}\right) .
\end{aligned}
$$

Note that the singular term in $p_{b}$ on $\phi_{2} \rightarrow \pi-\phi_{2}$ and therefore vanishes on performing the angular integration over $\phi_{2}$ using the $i \epsilon$ prescription. Therefore one is left with the following expression

$$
\begin{aligned}
\lim _{p_{b}, k_{y} \rightarrow 0} \frac{-A_{2}}{i p_{b} i k_{y}}=\frac{2 e^{3}}{8(2 \pi)^{5}} & \sum_{t= \pm} \int d p^{\prime} d \phi_{1} d \phi_{2} d \phi_{3} d \phi_{4} \times \\
& \times \frac{\sin \phi_{1}}{\cos ^{2} \phi_{1}} \frac{\sin ^{2} \phi_{2}}{\cos ^{2} \phi_{2}} \sin \phi_{3} t f\left(\left|p^{\prime}\right|-t e \mu_{-}\right) .
\end{aligned}
$$

The angular integration using the $i \epsilon$ prescription can be performed using the following results

$$
\int_{0}^{\pi} \frac{\sin \phi}{\cos ^{2} \phi}=-2, \quad \int_{0}^{\pi} \frac{\sin ^{2} \phi}{\cos ^{2} \phi}=-\pi, \quad \int_{0}^{\pi} \sin \phi=2 .
$$

Substituting these values for the angular integrations we obtain

$$
\begin{aligned}
\lim _{p_{b}, k_{y} \rightarrow 0} \frac{-A_{2}}{i p_{b} i k_{y}} & =\int_{0}^{\infty} d p^{\prime} \frac{e^{3}}{16 \pi^{3}}\left[f\left(p^{\prime}-e \mu_{-}\right)-f\left(p^{\prime}+e \mu_{-}\right)\right] \\
& =\frac{e^{4} \mu_{-}}{16 \pi^{3}} .
\end{aligned}
$$

Proceeding in a similar manner, we find that when we sum over pairs in $\{t, u, v\}$ as discussed earlier in term $A_{3}$ it vanishes. The term $A_{4}$ also vanishes due to the same reason. Now the exchange term in (B.5) also contributes equally. Therefore using the transport coefficient in the Euclidean theory is given by

$$
\tilde{\zeta}_{1}^{(6)}=\frac{3 e^{4}}{64 \pi^{3}} \mu_{-} .
$$

Note that from (4.3) the transport coefficient is $3 / 8$ times the $\langle j j j\rangle$ correlator.

\section{B.2 Contributions to $\zeta_{2}^{(6)}$}

After performing Wick contractions on the correlator corresponding to this transport coefficient we obtain

$$
\begin{gathered}
\left\langle j^{a}(p+k) j^{x}(-k) T^{\tau z}(-p)\right\rangle=i \frac{e^{2}}{2^{4} \beta} \sum_{m} \int \frac{d^{5} p^{\prime}}{(2 \pi)^{5}} \sum_{t, v, u= \pm} \operatorname{Tr}\left(\left[\gamma^{a} \gamma^{\alpha} \gamma^{x} \gamma^{\beta}\left(\gamma^{\tau} i p_{z}^{\prime}+i \omega_{-} \gamma^{z}\right) \gamma^{\nu} P_{+}\right] \times\right. \\
\left.\times \Delta_{t}\left(i \omega_{-}, p^{\prime}+p+k\right)\left(\hat{p}^{\prime}+\hat{p}+\hat{k}\right)_{(\alpha, t)} \Delta_{u}\left(i \omega_{-}, p^{\prime}+p\right)\left(\hat{p}^{\prime}+\hat{p}\right)_{(\beta, u)} \Delta_{v}\left(i \omega_{-}, p^{\prime}\right) \hat{p}_{(\nu, v)}^{\prime}\right)
\end{gathered}
$$




$$
\begin{aligned}
& +i \frac{e^{2}}{2^{4} \beta} \sum_{m} \int \frac{d^{5} p^{\prime}}{(2 \pi)^{5}} \sum_{t, v, u= \pm}\left(\operatorname{Tr}\left[\left(\gamma^{\tau} i p_{z}^{\prime}+i \omega_{-} \gamma^{z}\right) \gamma^{\alpha} \gamma^{x} \gamma^{\beta} \gamma^{a} \gamma^{\nu} P_{+}\right] \times\right. \\
& \left.\quad \times \Delta_{t}\left(i \omega_{-}, p^{\prime}-p\right)\left(\hat{p}^{\prime}-\hat{p}\right)_{(\alpha, t)} \Delta_{u}\left(i \omega_{-}, p^{\prime}-p-k\right)\left(\hat{p}^{\prime}-p-k\right)_{(\beta, u)} \Delta_{v}\left(i \omega_{-}, p^{\prime}\right) \hat{p}_{(\nu, v)}^{\prime}\right) .
\end{aligned}
$$

The second term in the curved brackets is obtained by the the exchange Wick contraction. Examining the terms we see that we need to evaluate the following integrals

$$
\begin{aligned}
A_{1}= & i^{2} \frac{e^{2}}{2^{4} \beta} \sum_{m} \int \frac{d^{5} p^{\prime}}{(2 \pi)^{5}} \sum_{t, v, u= \pm} \operatorname{Tr}\left[\gamma^{a} \gamma^{\alpha} \gamma^{x} \gamma^{\beta} \gamma^{\tau} \gamma^{\nu} P_{+}\right] p_{z}^{\prime} \times \\
& \times \Delta_{t}\left(i \omega_{-}, p^{\prime}+p+k\right)\left(\hat{p}^{\prime}+\hat{p}+\hat{k}\right)_{(\alpha, t)} \Delta_{u}\left(i \omega_{-}, p^{\prime}+p\right)\left(\hat{p}^{\prime}+\hat{p}\right)_{(\beta, u)} \Delta_{v}\left(i \omega_{-}, p^{\prime}\right) \hat{p}_{(\nu, v)}^{\prime}, \\
A_{2}= & i \frac{e^{2}}{2^{4} \beta} \sum_{m} \int \frac{d^{5} p_{1}}{(2 \pi)^{5}} \sum_{t, v, u= \pm} \operatorname{Tr}\left[\gamma^{a} \gamma^{\alpha} \gamma^{x} \gamma^{\beta} \gamma^{z} \gamma^{\nu} P_{+}\right] i \omega_{-} \times \\
& \times \Delta_{t}\left(i \omega_{-}, p^{\prime}+p+k\right)\left(\hat{p}^{\prime}+\hat{p}+\hat{k}\right)_{(\alpha, t)} \Delta_{u}\left(i \omega_{-}, p^{\prime}+p\right)\left(\hat{p}^{\prime}+\hat{p}\right)_{(\beta, u)} \Delta_{v}\left(i \omega_{-}, p^{\prime}\right) \hat{p}_{(\nu, v)}^{\prime} .
\end{aligned}
$$

Evaluating the trace over the $\gamma$ matrices, we end up with

$$
\begin{aligned}
A_{1}= & i \frac{e^{2}}{2^{4} \beta} \sum_{\omega_{m}} \int \frac{d^{5} p^{\prime}}{(2 \pi)^{5}} \sum_{t, v, u= \pm} \frac{4 t u v k_{y} p_{b} p_{z}^{\prime 2}}{E_{p^{\prime}} E_{p^{\prime}+p+k} E_{p^{\prime}+p}} \times \\
& \times \Delta_{t}\left(i \omega_{-}, p^{\prime}+p+k\right) \Delta_{u}\left(i \omega_{-}, p^{\prime}+p\right) \Delta_{v}\left(i \omega_{-}, p^{\prime}\right) \\
A_{2}= & i \frac{e^{2}}{2^{4} \beta} \sum_{\omega_{m}} \int \frac{d^{5} p^{\prime}}{(2 \pi)^{5}} \sum_{t, v, u= \pm}\left(\frac{4 t u\left(k_{y} p_{b}^{\prime}+p_{b} k_{y}\right)}{E_{p^{\prime}+p+k} E_{p^{\prime}+p}}-\frac{4 u v p_{y}^{\prime} p_{b}}{E_{p^{\prime}} E_{p^{\prime}+p}}\right. \\
& \left.-\frac{4 t v\left(k_{y} p_{1 b}-p_{b} p_{y}^{\prime}\right)}{E_{p^{\prime}+p+k} E_{p^{\prime}}}\right) i \omega_{-} \Delta_{t}\left(i \omega_{-}, p^{\prime}+p+k\right) \Delta_{u}\left(i \omega_{-}, p^{\prime}+p\right) \Delta_{v}\left(i \omega_{-}, p^{\prime}\right)
\end{aligned}
$$

The sum over Matsubara frequencies in $A_{1}$ is performed using (B.9). To perform the sum in $A_{2}$, we use the following

$$
\begin{aligned}
& \frac{1}{\beta} \sum_{m} i \omega_{-} \Delta_{t}\left(i \omega_{-}, p^{\prime}+p+k\right) \Delta_{u}\left(i \omega_{-}, p^{\prime}+p\right) \Delta_{v}\left(i \omega_{-}, p^{\prime}\right) \\
& =\frac{E_{p^{\prime}+p+k} f\left(E_{p^{\prime}+p+k}-t e \mu_{-}\right)}{\left(t E_{p^{\prime}+p+k}-v E_{p^{\prime}}\right)\left(t E_{p^{\prime}+p+k}-u E_{p^{\prime}+p}\right)}+\frac{E_{p^{\prime}+p} f\left(E_{p^{\prime}+p}-u e \mu_{-}\right)}{\left(u E_{p^{\prime}+p}-t E_{p^{\prime}+p+k}\right)\left(u E_{p^{\prime}+p}-v E_{p^{\prime}}\right)} \\
& \quad+\frac{E_{p^{\prime}} f\left(E_{p^{\prime}}-v e \mu_{-}\right)}{\left(v E_{p^{\prime}}-t E_{p^{\prime}+p+k}\right)\left(v E_{p^{\prime}}-u E_{p^{\prime}+p}\right)} .
\end{aligned}
$$

We can now proceed as discussed in B.1 to perform the sum over $\{t, u, v\}$ and then change variable of integrations. Finally after performing the angular integrals using the $i \epsilon$ prescription, we obtain

$$
\begin{aligned}
\lim _{p_{b}, k_{y} \rightarrow 0} \frac{-A_{1}}{i p_{b} i k_{y}} & =i \frac{e^{2}}{32 \pi^{3}} \sum_{t= \pm} \int_{0}^{\infty} d p^{\prime} p^{\prime} f\left(p^{\prime}-t e \mu_{-}\right) \\
\lim _{p_{b}, k_{y} \rightarrow 0} \frac{-A_{2}}{i p_{b} i k_{y}} & =i \frac{e^{2}}{32 \pi^{3}} \sum_{t= \pm} \int_{0}^{\infty} d p^{\prime} p p^{\prime} f\left(p^{\prime}-t e \mu_{-}\right) .
\end{aligned}
$$


The exchange term in (B.18) also contributes an equal amount to the correlator. Therefore, we have

$$
\begin{aligned}
\lim _{p_{b}, k_{y} \rightarrow 0}-\frac{j^{a}(k+p) j^{x}(-k) T^{t z}(-p)}{i p_{b} i k_{y}} & =\lim _{p_{b}, k_{y} \rightarrow 0}-2 \frac{A_{1}+A_{2}}{i p_{b} i k_{y}} \\
& =i \frac{e^{2}}{8 \pi^{3}} \sum_{t= \pm} \int_{0}^{\infty} d p^{\prime} p^{\prime} f\left(p^{\prime}-t e \mu_{-}\right) \\
& =i \frac{e^{2}}{16 \pi^{3}}\left(e^{2}\left(\mu_{-}\right)^{2}+\frac{\pi^{2} T^{2}}{3}\right) .
\end{aligned}
$$

\section{B.3 Contributions to $\zeta_{3}^{(6)}$}

The correlator corresponding to the transport coefficient $\zeta_{3}^{(6)}$ also involves contact terms. We will first evaluate the contribution from Wick contractions of the flat space currents in the correlator $\left\langle j_{f l}^{a} T_{f l}^{t x} T_{f l}^{t z}\right\rangle$. We then proceed to evaluate the contribution from the contact term.

$$
\begin{aligned}
& \left\langle j_{f l}^{a}(p+k) T_{f l}^{\tau x}(-k) T_{f l}^{\tau z}(-p)\right\rangle \\
& =-\frac{e}{32 \beta} \sum_{m} \int \frac{d^{5} p^{\prime}}{(2 \pi)^{5}} \sum_{t, v, u= \pm} \operatorname{Tr}\left[\gamma^{a} \gamma^{\alpha}\left(\gamma^{\tau} i p_{x}^{\prime}+\gamma^{x} i \omega_{-}\right) \gamma^{\beta}\left(\gamma^{\tau} i p_{p_{z}}+\gamma^{z} i \omega_{-}\right) \gamma^{\nu} P_{+}\right] \\
& \quad \times\left(\hat{p}^{\prime}+\hat{p}+\hat{k}\right)_{(t, \alpha)}\left(\hat{p}^{\prime}+\hat{p}\right)_{(u, \beta)} p_{(v, \nu)}^{\prime} \Delta_{t}\left(i \omega_{-}, p^{\prime}+k+p\right) \Delta_{u}\left(i \omega_{-}, p^{\prime}+p\right) \Delta_{v}\left(i \omega_{-}, p^{\prime}\right) \\
& \quad+(\text { Exchange term). }
\end{aligned}
$$

Among the four possible traces it can be seen that only 3 of them contribute, they are given by

$$
\begin{aligned}
A_{1}= & -\frac{i e}{32 \beta} \sum_{m} \int \frac{d^{5} p^{\prime}}{(2 \pi)^{5}} \sum_{t, v, u= \pm} \operatorname{Tr}\left[\gamma^{a} \gamma^{\alpha} \gamma^{\tau} \gamma^{\beta} \gamma^{z} \gamma^{\nu} P_{+}\right] p_{x}^{\prime} i \omega_{-} \\
& \times\left(\hat{p}^{\prime}+\hat{p}+\hat{k}\right)_{(t, \alpha)}\left(\hat{p}^{\prime}+\hat{p}\right)_{(u, \beta)} \hat{p}_{(v, \nu)}^{\prime} \Delta_{t}\left(i \omega_{-}, p^{\prime}+k+p\right) \Delta_{u}\left(i \omega_{-}, p^{\prime}+p\right) \Delta_{v}\left(i \omega_{-}, p^{\prime}\right) \\
& +(\text { Exchange term), } \\
A_{2}= & -\frac{i e}{32 \beta} \sum_{m} \int \frac{d^{5} p^{\prime}}{(2 \pi)^{5}} \sum_{t, v, u= \pm} \operatorname{Tr}\left[\gamma^{a} \gamma^{\alpha} \gamma^{x} \gamma^{\beta} \gamma^{\tau} \gamma^{\nu} P_{+}\right] p_{z}^{\prime} i \omega_{-} \\
& \quad \times\left(\hat{p}^{\prime}+\hat{p}+\hat{k}\right)_{(t, \alpha)}\left(\hat{p}^{\prime}+\hat{p}\right)_{(u, \beta)} \hat{p}_{(v, \nu)}^{\prime} \Delta_{t}\left(i \omega_{-}, p^{\prime}+k+p\right) \Delta_{u}\left(i \omega_{-}, p^{\prime}+p\right) \Delta_{v}\left(i \omega_{-}, p^{\prime}\right) \\
& +(\text { Exchange term) } \\
A_{3}= & \frac{-e}{32 \beta} \sum_{m} \int \frac{d^{5} p^{\prime}}{(2 \pi)^{5}} \sum_{t, v, u= \pm} \operatorname{Tr}\left[\gamma^{a} \gamma^{\alpha} \gamma^{x} \gamma^{\beta} \gamma^{z} \gamma^{\nu} P_{+}\right]\left(i \omega_{-}\right)^{2} \\
& \times\left(\hat{p}^{\prime}+\hat{p}+\hat{k}\right)_{(t, \alpha)}\left(\hat{p}^{\prime}+\hat{p}\right)_{(u, \beta)} \hat{p}_{(v, \nu)}^{\prime} \Delta_{t}\left(i \omega_{-}, p^{\prime}+k+p\right) \Delta_{u}\left(i \omega_{-}, p^{\prime}+p\right) \Delta_{v}\left(i \omega_{-}, p^{\prime}\right) \\
& +(\text { Exchange term). }
\end{aligned}
$$

Now we follow the methods adopted to evaluate the previous correlators. We first perform the traces in each of them and follow that up with the Matsubara sum and then take the limit, $p_{b}, k_{y} \rightarrow 0$. Lastly we perform the angular integrals with $i \epsilon$ trick. We find that the terms $A_{1}$ and $A_{2}$ contribute equally and they are given by

$$
A_{1}=\frac{-e}{32 \beta} \sum_{m} \int \frac{d^{5} p^{\prime}}{(2 \pi)^{5}} \sum_{t, v, u= \pm} \frac{4 t u v k_{y} p_{b} p_{x}^{\prime 2} i \omega_{-}}{E_{p^{\prime}} E_{p^{\prime}+p+k} E_{p^{\prime}+p}} \Delta_{t}\left(p^{\prime}+k+p\right) \Delta_{u}\left(p^{\prime}+p\right) \Delta_{v}\left(p^{\prime}\right)
$$




$$
\begin{aligned}
& +(\text { Exchange term }), \\
A_{2}= & \frac{-e}{32 \beta} \sum_{m} \int \frac{d^{5} p^{\prime}}{(2 \pi)^{5}} \sum_{t, v, u= \pm} \frac{4 t u v k_{y} p_{b} p_{z}^{2} i \omega_{-}}{E_{p^{\prime}} E_{p^{\prime}+p+k} E_{p^{\prime}+p}} \Delta_{t}\left(p^{\prime}+k+p\right) \Delta_{u}\left(p^{\prime}+p\right) \Delta_{v}\left(p^{\prime}\right) \\
& +(\text { Exchanged term }) .
\end{aligned}
$$

The Matsubara sums are performed using equation (B.21). After a change of variables of integration and taking the external momenta to zero, we obtain

$$
\lim _{p_{b}, k_{y} \rightarrow 0} \frac{-A_{1}}{i p_{b} i k_{y}}=-\frac{2 e}{64 \pi^{3}} \sum_{t= \pm} \int_{0}^{\infty} d p^{\prime} t p^{\prime 2} f\left(p^{\prime}-t e \mu_{-}\right) .
$$

Here again we have used the $i \epsilon$ prescription to evaluate the integrals. The factor of 2 results from taking into account the equal contribution of the exchange term. Evaluating the $A_{3}$ term involves performing the following Matsubara sum

$$
\begin{aligned}
& \frac{1}{\beta} \sum_{m}\left(i \omega_{-}\right)^{2} \Delta_{t}\left(i \omega_{-}, p^{\prime}+p+k\right) \Delta_{u}\left(i \omega_{-}, p^{\prime}+p\right) \Delta_{v}\left(i \omega_{-}, p_{1}\right) \\
& =\frac{t E_{p^{\prime}+p+k}^{2} f\left(E_{p^{\prime}+p+k}-t e \mu_{-}\right)}{\left(t E_{p^{\prime}+p+k}-v E_{p^{\prime}}\right)\left(t E_{p^{\prime}+p+k}-u E_{p^{\prime}+p}\right)}+\frac{u E_{p^{\prime}+p}^{2} f\left(E_{p^{\prime}+p}-u e \mu_{-}\right)}{\left(u E_{p^{\prime}+p}-t E_{p^{\prime}+p+k}\right)\left(u E_{p^{\prime}+p}-v E_{p^{\prime}}\right)} \\
& \quad+\frac{v E_{p^{\prime}}^{2} f\left(E_{p^{\prime}}-v e \mu_{-}\right)}{\left(v E_{p^{\prime}}-t E_{p^{\prime}+p+k}\right)\left(v E_{p^{\prime}}-u E_{p^{\prime}+p}\right)}, \\
& \equiv \mathcal{N} .
\end{aligned}
$$

Performing the trace over the $\gamma$ matrices and substituting the Matsubara sum $\mathcal{N}$, we obtain

$$
\begin{aligned}
A_{3}= & \frac{e}{32 \beta} \sum_{m} \int \frac{d^{5} p^{\prime}}{(2 \pi)^{5}} \sum_{t, v, u= \pm}\left(\frac{4 t u\left(k_{y} p_{b}^{\prime}+p_{b} k_{y}\right)}{E_{p^{\prime}+p+k} E_{p^{\prime}+p}}-\frac{4 u v p_{y}^{\prime} p_{b}}{E_{p^{\prime}} E_{p^{\prime}+p}}\right. \\
& \left.-\frac{4 t v\left(k_{y} p_{b}^{\prime}-p_{b} p_{y}^{\prime}\right)}{E_{p^{\prime}+p+k} E_{p^{\prime}}}\right) \times \mathcal{N} \\
& + \text { (Exchange term). }
\end{aligned}
$$

Now summing over $\{t, u, v\}$, making appropriate shifts in the integrations and taking the zero momentum limit, we get

$$
\lim _{p_{b}, k_{y} \rightarrow 0} \frac{-A_{3}}{i p_{b} i k_{y}}=-\frac{2 e}{64 \pi^{3}} \sum_{t= \pm} \int_{0}^{\infty} d p^{\prime} t p^{2} f\left(p^{\prime}-t e \mu_{-}\right) .
$$

Therefore the putting terms $A_{1}, A_{2}, A_{3}$ together we see that the contributions from the Wick contractions of the flat space currents is

$$
\lim _{p_{b}, k_{y} \rightarrow 0}-\frac{\left\langle j_{f l}^{a}(-p-k) T_{f l}^{t x}(k) T_{f l}^{t z}(p)\right\rangle}{i p_{b} i k_{y}}=-\frac{3 e}{32 \pi^{3}} \sum_{t= \pm} \int_{0}^{\infty} d p^{\prime} p^{\prime 2} t f\left(p^{\prime}-t e \mu_{-}\right) .
$$

We will now obtain the contribution from the contact terms. For this we first Fourier transform the interaction $S_{E}^{2}$ given in (4.11), this yields

$$
\frac{\delta S_{E}^{(2)}}{\delta h_{x \tau}(k) \delta h_{z, \tau}(p)}=-\frac{i}{16 \beta} \sum_{m} \int \frac{d^{5} p^{\prime}}{(2 \pi)^{5}} \psi^{\dagger}\left(\omega_{m}, p+k+p^{\prime}\right)\left(p_{b} \gamma^{b} \gamma^{x} \gamma^{z}+k_{y} \gamma^{y} \gamma^{z} \gamma^{x}\right) P_{-} \psi\left(\omega_{m}, p^{\prime}\right)
$$


Considering the Wick contraction of the above contact term along with the flat space charge current, results in the following two terms

$$
\begin{aligned}
\frac{-1}{i p_{b} i k_{y}} B & =B_{1}+B_{2} \\
B_{1} & =\frac{e}{16 k_{y}} \frac{1}{\beta} \sum_{m} \int \frac{d^{5} p^{\prime}}{(2 \pi)^{5}} \operatorname{Tr}\left[\gamma^{a} S\left(\omega_{m}, p+k+p^{\prime}\right) \gamma^{b} \gamma^{x} \gamma^{z} S\left(\omega_{m}, p^{\prime}\right) P_{+}\right], \\
B_{2} & =\frac{e}{16 p_{b}} \frac{1}{\beta} \sum_{m} \int \frac{d^{5} p^{\prime}}{(2 \pi)^{5}} \operatorname{Tr}\left[\gamma^{a} S\left(\omega_{m}, p+k+p^{\prime}\right) \gamma^{b} \gamma^{z} \gamma^{x} S\left(\omega_{m}, p^{\prime}\right) P_{+}\right]
\end{aligned}
$$

We proceed to evaluate $B_{1}$. Substituting the expression for thermal propagators we obtain,

$$
\begin{aligned}
\lim _{p_{b}, k_{y} \rightarrow 0} \lim _{p_{0}, k_{0} \rightarrow 0} B_{1}= & \lim _{p_{b}, k_{y} \rightarrow 0} \frac{-e}{16 k_{y}} \frac{1}{\beta} \sum_{m} \int \frac{d^{5} p^{\prime}}{(2 \pi)^{5}} \sum_{t, u= \pm} \operatorname{Tr}\left[\gamma^{a} \gamma^{\alpha} \gamma^{b} \gamma^{x} \gamma^{z} \gamma^{\beta} P_{+}\right] \\
& \times \frac{1}{4}\left(\hat{p}^{\prime}+\hat{p}+\hat{k}\right)_{(t, \alpha)} \hat{p}^{\prime}{ }_{(u, \beta)} \Delta_{t}\left(i \omega_{-}, p^{\prime}+p+k\right) \Delta_{u}\left(i \omega_{-}, p^{\prime}\right), \\
= & \lim _{p_{b}, k_{y} \rightarrow 0} \frac{e}{16} \sum_{t, u= \pm} \int \frac{d^{5} p^{\prime}}{(2 \pi)^{5}} \frac{t}{E_{p^{\prime}+p+k}} \frac{t f\left(E_{p^{\prime}+p+k}-t e \mu_{-}\right)-u f\left(E_{p^{\prime}}-u e \mu_{-}\right)}{t E_{p^{\prime}+p+k}-u E_{p^{\prime}}}, \\
= & \frac{e}{4(2 \pi)^{5}} \int d p^{\prime} d \phi_{1} d \phi_{2} d \phi_{3}(2 \pi) \sum_{t= \pm} p^{\prime 4} \sin ^{3} \phi_{1} \sin ^{2} \phi_{2} \sin \phi_{3} \\
& \quad \times\left(-\frac{1}{2 p_{b} p^{\prime} \cos \phi_{1}}+\frac{1}{4 p^{\prime 2} \cos ^{2} \phi_{1}}\right)+O\left(k_{y} / p_{b}, p_{b}\right) .
\end{aligned}
$$

To obtain the last equality we have summed over $\{t, u\}$ and then taken the external momenta to zero. We have taken $k_{y} \rightarrow 0$ first and then taken the limit $p_{b} \rightarrow 0$. But the final result for the non-singular term is independent of the order of limits. Also note that the singular term in $p_{b}$ is odd under $\phi_{1} \rightarrow \pi-\phi_{1}$. Therefore it vanishes on performing the angular integration over $\phi_{1}$. If the order of limit is interchanged the resulting singular terms in $k_{y}$ also vanish on angular integration and the final result for the non-singular term is invariant. The angular integration in this term can be evaluated easily by the $i \epsilon$ prescription,

$$
\int_{0}^{\pi} \frac{\sin ^{3} \phi_{1}}{\cos ^{2} \phi_{1}}=-4
$$

Substituting this result into $B_{1}$ we obtain

$$
\lim _{p_{b}, k_{y} \rightarrow 0} B_{1}=-\frac{e}{64 \pi^{3}} \sum_{t= \pm} \int_{0}^{\infty} d p^{\prime} t p^{\prime 2} f\left(p^{\prime}-t e \mu_{-}\right) .
$$

Going through a similar analysis on $B_{2}$ it can be shown that the result for $B_{2}$ is equal to that of $B_{1}$. Thus the total contribution from the contact term is given by

$$
\lim _{p_{b}, k_{y} \rightarrow 0} B=-\frac{2 e}{64 \pi^{3}} \sum_{t= \pm} \int_{0}^{\infty} d p^{\prime} t p^{\prime 2} f\left(p^{\prime}-t e \mu_{-}\right) .
$$

Therefore putting all the terms from $A$ and $B$ together we obtain the final result for the correlator of interest to be

$$
\begin{aligned}
\frac{-1}{i p_{b} i k_{y}}\left\langle j^{a}(p+k) T^{\tau x}(-k) T^{\tau z}(-p)\right\rangle & =\frac{-e}{8 \pi^{3}} \sum_{t= \pm} \int_{0}^{\infty} d p^{\prime} t p^{2} f\left(p_{1}-t e \mu_{-}\right) \\
& =\frac{-e}{24 \pi^{3}}\left(e^{3} \mu_{-}^{3}+e \pi^{2} T^{2} \mu_{-}\right) .
\end{aligned}
$$




\section{B.4 Contributions to $\lambda_{3}^{(6)}$}

There are two contributions to this correlator, one from the direct Wick contractions of the flat space currents and the other from the contact terms. Let us first evaluate the former contribution, performing Wick contractions leads to

$$
\begin{aligned}
& \left\langle T_{f l}^{\tau a}(p+k) T_{f l}^{\tau x}(-k) T_{f l}^{\tau z}(-p)\right\rangle=\frac{-i}{\beta} \sum_{m} \frac{1}{64} \int \frac{d^{5} p^{\prime}}{(2 \pi)^{5}} \times \\
& \times\left(\operatorname{Tr}\left[\gamma^{\tau} i p_{a}^{\prime}+\gamma^{a} i \omega_{-}\right) \gamma^{\alpha}\left(\gamma^{\tau} i p_{x}^{\prime}+\gamma^{x} i \omega_{-}\right) \gamma^{\beta}\left(\gamma^{\tau} i p_{z}^{\prime}+\gamma^{z} i \omega_{-}\right) \gamma^{\nu} P_{+}\right] \\
& \left.\times \Delta_{t}\left(i \omega_{-}, p^{\prime}+p+k\right) \Delta_{u}\left(i \omega_{-}, p^{\prime}+p\right) \Delta_{v}\left(i \omega_{-}, p^{\prime}\right)\left(\hat{p}^{\prime}+\hat{p}+\hat{k}\right)_{(t, \alpha)}\left(\hat{p}^{\prime}+\hat{p}\right)_{(u, \beta)} \hat{p}_{(v, \nu)}^{\prime}\right) \\
& +(\text { exchange term }) .
\end{aligned}
$$

Expanding the above express it can be seen that there are 4 terms which are non-vanishing these are given by

$$
\begin{aligned}
A_{1}= & -\frac{1}{64 \beta} \sum_{m} \int \frac{d^{5} p^{\prime}}{(2 \pi)^{5}} \operatorname{Tr}\left[\gamma^{\tau} \gamma^{\alpha} \gamma^{x} \gamma^{\beta} \gamma^{z} \gamma^{\nu} P_{+}\right] p_{a}^{\prime} \omega_{-}^{2} \times \\
& \times \Delta_{t}\left(i \omega_{-}, p^{\prime}+p+k\right) \Delta_{u}\left(i \omega_{-}, p^{\prime}+p\right)_{v}\left(i \omega_{-}, p^{\prime}\right)\left(\hat{p}^{\prime}+\hat{p}+\hat{k}\right)_{(t, \alpha)}\left(\hat{p}^{\prime}+\hat{p}\right)_{(u, \beta)} \hat{p}_{(v, \gamma)}^{\prime} \\
& +(\text { exchange term }), \\
A_{2}= & -\frac{1}{64 \beta} \sum_{m} \int \frac{d^{5} p^{\prime}}{(2 \pi)^{5}} \operatorname{Tr}\left[\gamma^{a} \gamma^{\alpha} \gamma^{x} \gamma^{\beta} \gamma^{\tau} \gamma^{\nu} P_{+}\right] p_{z}^{\prime} \omega_{-}^{2} \times \\
& \times \Delta_{t}\left(i \omega_{-} p^{\prime}+p+k\right) \Delta_{u}\left(i \omega_{-}, p^{\prime}+p\right)_{v}\left(i \omega_{-}, p^{\prime}\right)\left(\hat{p}^{\prime}+\hat{p}+\hat{k}\right)_{(t, \alpha)}\left(\hat{p}^{\prime}+\hat{p}\right)_{(u, \beta)} \hat{p}_{(v, \nu)}^{\prime} \\
& +(\text { exchange term }), \\
A_{3}= & -\frac{1}{64 \beta} \sum_{m} \int \frac{d^{5} p^{\prime}}{(2 \pi)^{5}} \operatorname{Tr}\left[\gamma^{a} \gamma^{\alpha} \gamma^{\tau} \gamma^{\beta} \gamma^{z} \gamma^{\nu} P_{+}\right] p_{x}^{\prime} \omega_{-}^{2} \times \\
& \times \Delta_{t}\left(i \omega_{-}, p^{\prime}+p+k\right) \Delta_{u}\left(i \omega_{-}, p^{\prime}+p\right) \Delta_{v}\left(i \omega_{-}, p^{\prime}\right)\left(\hat{p}^{\prime}+\hat{p}+\hat{k}\right)_{(t, \alpha)}\left(\hat{p}^{\prime}+\hat{p}\right)_{(u, \beta)} \hat{p}_{(v, \nu)} \\
& +(\operatorname{exchange~term)}, \\
A_{4}= & -\frac{1}{64 \beta} \sum_{m} \int \frac{d^{5} p^{\prime}}{(2 \pi)^{5}} \operatorname{Tr}\left[\gamma^{a} \gamma^{\alpha} \gamma^{x} \gamma^{\beta} \gamma^{z} \gamma^{\nu} P_{+}\right] \omega_{-}^{3} \\
& \times \Delta_{t}\left(i \omega_{-}, p^{\prime}+p+k\right) \Delta_{u}\left(i \omega_{-}, p^{\prime}+p\right) \Delta_{v}\left(i \omega_{-}, p^{\prime}\right)\left(\hat{p}^{\prime}+\hat{p}+\hat{k}\right)_{t, \alpha}\left(\hat{p}^{\prime}+\hat{p}\right)_{u, \beta} \hat{p}_{(v, \nu)} \\
& +(\operatorname{exchange} \operatorname{term}) .
\end{aligned}
$$

We find that contribution of the terms $A_{1}, A_{2}$ and $A_{3}$ are equal. Furthermore the contribution of the direct Wick contraction is equal to that of the exchange term in each $A_{1}, A_{2}, A_{3}, A_{4}$. After performing the trace over $\gamma$ matrices, we find that $A_{1}$ is given by

$$
\begin{aligned}
A_{1}= & \frac{2 i}{64 \beta} \sum_{m} \int \frac{d^{5} p^{\prime}}{(2 \pi)^{5}} \sum_{t, u, v= \pm} \frac{4 t u v p_{a}^{\prime 2}}{E_{p^{\prime}+p+p} E_{p^{\prime}+p} E_{p^{\prime}}} \omega_{-}^{2} \times \\
& \times \Delta_{t}\left(i \omega_{-}, p^{\prime}+p+k\right) \Delta_{u}\left(i \omega_{-}, p^{\prime}+p\right) \Delta_{v}\left(i \omega_{-}, p^{\prime}\right) .
\end{aligned}
$$


Here the factor of 2 is due to the contribution of the exchange term. Proceeding as in discussed in the previous examples we obtain

$$
\lim _{p_{b}, k_{y} \rightarrow 0}-\frac{A_{1}}{i p_{b} i k_{y}}=-\frac{2 i}{128 \pi^{3}} \sum_{t= \pm} \int_{0}^{\infty} d p^{\prime} p^{\prime 3} f\left(p^{\prime}-t e \mu_{-}\right) .
$$

Since the contribution of $A_{2}$ and $A_{3}$ is identical to that of $A_{1}$ we have

$$
\lim _{p_{b}, k_{y} \rightarrow 0}-\frac{A_{1}+A_{2}+A_{3}}{i p_{b} i k_{y}}=-\frac{6 i}{128 \pi^{3}} \sum_{t= \pm} \int_{0}^{\infty} d p^{\prime} p^{\prime 3} f\left(p^{\prime}-t e \mu_{-}\right) .
$$

To evaluate the term $A_{4}$ we need to perform the matsubara sum which is given by

$$
\begin{aligned}
& \frac{1}{\beta} \sum_{m}\left(i \omega_{-}\right)^{3} \Delta_{t}\left(i \omega_{-}, p^{\prime}+p+k\right) \Delta_{u}\left(i \omega_{-}, p^{\prime}+p\right) \Delta_{v}\left(i \omega_{-}, p^{\prime}\right) \\
& =\frac{E_{p^{\prime}+p+k}^{3} f\left(E_{p^{\prime}+p+k}-t e \mu_{-}\right)}{\left(t E_{p^{\prime}+p+k}-v E_{p^{\prime}}\right)\left(t E_{p^{\prime}+p+k}-u E_{p^{\prime}+p}\right)}+\frac{E_{p^{\prime}+p}^{3} f\left(E_{p^{\prime}+p}-u e \mu_{-}\right)}{\left(u E_{p^{\prime}+p}-t E_{p^{\prime}+p+k}\right)\left(u E_{p^{\prime}+p}-v E_{p^{\prime}}\right)} \\
& \quad+\frac{E_{p^{\prime}}^{3} f\left(E_{p^{\prime}}-v e \mu_{-}\right)}{\left(v E_{p^{\prime}}-t E_{p^{\prime}+p+k}\right)\left(v E_{p^{\prime}}-u E_{p^{\prime}+p}\right)} .
\end{aligned}
$$

At the end of the usual manipulations, we obtain

$$
\lim _{p_{b}, k_{y} \rightarrow 0}-\frac{A_{4}}{i p_{b} i k_{y}}=-\frac{2 i}{128 \pi^{3}} \sum_{t= \pm} \int_{0}^{\infty} d p^{\prime} p^{\prime 3} f\left(p^{\prime}-t e \mu_{-}\right) .
$$

Therefore the contribution from the Wick contractions of the flat space current is given by

$$
\lim _{p_{b}, k_{y} \rightarrow 0}-\frac{\left\langle T^{\tau a}(p+k) T^{\tau x}(-k) T^{\tau z}(-p)\right\rangle}{i p_{b} i k_{y}}=\frac{-8 i}{128 \pi^{3}} \sum_{t= \pm} \int d p^{\prime} p^{\prime 3} f\left(p^{\prime}-t e \mu_{-}\right) .
$$

Now lets examine the contact terms. As seen in the equation (4.37), there are 3 contact terms. We will evaluate one of them, it can be seen that the contributions from all the 3 contact terms are equal. Performing the Wick contraction of the contact term with the flat space stress tensor we obtain

$$
\begin{aligned}
\frac{-B_{1}}{i p_{b} i k_{y}}= & -\frac{1}{32 i \beta} \sum_{m} \int \frac{d^{5} p^{\prime}}{(2 \pi)^{5}}\left(\frac{1}{k_{y}} \operatorname{Tr}\left[\left(\gamma^{\tau} i p_{a}^{\prime}+\gamma^{a} i \omega_{-}\right) S\left(\omega_{m}, p^{\prime}+p+k\right)\left(\gamma^{b} \gamma^{x} \gamma^{z}\right) S\left(\omega_{m}, p^{\prime}\right) P_{+}\right]\right. \\
& \left.+\frac{1}{p_{b}} \operatorname{Tr}\left[\left(\gamma^{\tau} i p_{a}^{\prime}+\gamma^{a} i \omega_{-}\right) S\left(\omega_{m}, p^{\prime}+p+k\right)\left(\gamma^{y} \gamma^{z} \gamma^{x}\right) S\left(\omega_{m}, p^{\prime}\right) P_{+}\right]\right) .
\end{aligned}
$$

We then follow the same steps as before to evaluate this integral and get

$$
\lim _{p_{b}, k_{y} \rightarrow 0}-\frac{B_{1}}{i p_{b} i k_{y}}=\frac{-8 i}{3} \frac{1}{128 \pi^{3}} \sum_{t= \pm} \int_{0}^{\infty} d p^{\prime} p^{\prime 3} f\left(p^{\prime}-t e \mu_{-}\right) .
$$

The other two contact terms $B_{2}$ and $B_{3}$ also give the same amount of contribution. Therefore the total contribution from the contact terms is given by

$$
B=-\frac{8 i}{128 \pi^{3}} \sum_{t= \pm} \int_{0}^{\infty} d p^{\prime} p^{\prime 3} f\left(p^{\prime}-t e \mu_{-}\right) .
$$

Combing the terms $A$ and $B$ we see that the value for the three point function of interest is given by

$$
\lim _{p_{b}, k_{y} \rightarrow 0}-\frac{\left\langle T^{\tau a}(k+p) T^{\tau x}(-k) T^{\tau z}(-p)\right\rangle}{i p_{b} i k_{y}}=\frac{-16 i}{128 \pi^{3}} \sum_{t= \pm} \int_{0}^{\infty} d p^{\prime} p^{\prime 3} f\left(p^{\prime}-t e \mu_{-}\right) .
$$


Open Access. This article is distributed under the terms of the Creative Commons Attribution License (CC-BY 4.0), which permits any use, distribution and reproduction in any medium, provided the original author(s) and source are credited.

\section{References}

[1] S. Bhattacharyya, S. Lahiri, R. Loganayagam and S. Minwalla, Large rotating AdS black holes from fluid mechanics, JHEP 09 (2008) 054 [arXiv:0708.1770] [INSPIRE].

[2] J. Erdmenger, M. Haack, M. Kaminski and A. Yarom, Fluid dynamics of R-charged black holes, JHEP 01 (2009) 055 [arXiv:0809.2488] [INSPIRE].

[3] N. Banerjee, J. Bhattacharya, S. Bhattacharyya, S. Dutta, R. Loganayagam and P. Surowka, Hydrodynamics from charged black branes, JHEP 01 (2011) 094 [arXiv:0809.2596] [INSPIRE].

[4] D.T. Son and P. Surowka, Hydrodynamics with triangle anomalies, Phys. Rev. Lett. 103 (2009) 191601 [arXiv:0906.5044] [INSPIRE].

[5] N. Banerjee, S. Dutta, S. Jain, R. Loganayagam and T. Sharma, Constraints on anomalous fluid in arbitrary dimensions, JHEP 03 (2013) 048 [arXiv:1206.6499] [INSPIRE].

[6] K. Jensen, M. Kaminski, P. Kovtun, R. Meyer, A. Ritz and A. Yarom, Towards hydrodynamics without an entropy current, Phys. Rev. Lett. 109 (2012) 101601 [arXiv: 1203.3556] [INSPIRE].

[7] K. Jensen, R. Loganayagam and A. Yarom, Thermodynamics, gravitational anomalies and cones, JHEP 02 (2013) 088 [arXiv: 1207.5824] [INSPIRE].

[8] K. Landsteiner, E. Megias and F. Pena-Benitez, Gravitational anomaly and transport, Phys. Rev. Lett. 107 (2011) 021601 [arXiv:1103.5006] [INSPIRE].

[9] K. Landsteiner, E. Megias, L. Melgar and F. Pena-Benitez, Holographic gravitational anomaly and chiral vortical effect, JHEP 09 (2011) 121 [arXiv:1107.0368] [INSPIRE].

[10] K. Landsteiner, E. Megias and F. Pena-Benitez, Anomalous transport from Kubo formulae, Lect. Notes Phys. 871 (2013) 433 [arXiv:1207.5808] [INSPIRE].

[11] S. Golkar and D.T. Son, (Non)-renormalization of the chiral vortical effect coefficient, JHEP 02 (2015) 169 [arXiv: 1207.5806] [INSPIRE].

[12] K. Jensen, R. Loganayagam and A. Yarom, Chern-Simons terms from thermal circles and anomalies, JHEP 05 (2014) 110 [arXiv:1311.2935] [INSPIRE].

[13] L. Di Pietro and Z. Komargodski, Cardy formulae for SUSY theories in $d=4$ and $d=6$, JHEP 12 (2014) 031 [arXiv:1407.6061] [INSPIRE].

[14] M. Valle, Hydrodynamics in $1+1$ dimensions with gravitational anomalies, JHEP 08 (2012) 113 [arXiv: 1206.1538] [INSPIRE].

[15] S. Jain and T. Sharma, Anomalous charged fluids in $1+1 d$ from equilibrium partition function, JHEP 01 (2013) 039 [arXiv:1203.5308] [INSPIRE].

[16] R. Banerjee, P. Chakraborty, S. Dey, B.R. Majhi and A.K. Mitra, Two dimensional hydrodynamics with gauge and gravitational anomalies, Phys. Rev. D 89 (2014) 104013 [arXiv:1307.1313] [INSPIRE]. 
[17] R. Loganayagam, Anomaly induced transport in arbitrary dimensions, arXiv:1106.0277 [INSPIRE].

[18] R. Loganayagam and P. Surowka, Anomaly/transport in an ideal Weyl gas, JHEP 04 (2012) 097 [arXiv: 1201.2812] [INSPIRE].

[19] R. Loganayagam, Anomalies and the helicity of the thermal state, JHEP 11 (2013) 205 [arXiv: 1211.3850] [INSPIRE].

[20] C. Eling, Y. Oz, S. Theisen and S. Yankielowicz, Conformal anomalies in hydrodynamics, JHEP 05 (2013) 037 [arXiv: 1301.3170] [inSPIRE].

[21] D.E. Kharzeev and H.J. Warringa, Chiral magnetic conductivity, Phys. Rev. D 80 (2009) 034028 [arXiv:0907.5007] [INSPIRE].

[22] H.-U. Yee, Chiral magnetic and vortical effects in higher dimensions at weak coupling, Phys. Rev. D 90 (2014) 065021 [arXiv:1406.3584] [INSPIRE].

[23] L. Álvarez-Gaumé and E. Witten, Gravitational anomalies, Nucl. Phys. B 234 (1984) 269 [INSPIRE].

[24] J.R. David, M. Mahato, S. Thakur and S.R. Wadia, Hydrodynamics of R-charged D1-branes, JHEP 01 (2011) 014 [arXiv: 1008.4350] [InSPIRE]. 